Florida International University FIU Digital Commons

$11-10-2010$

\title{
Health Monitoring of Round Objects using Multiple Structural Health Monitoring Techniques
}

Gurjashan Singh

Florida International University, gsing005@fiu.edu

DOI: $10.25148 /$ etd.FI10122002

Follow this and additional works at: https://digitalcommons.fiu.edu/etd

\section{Recommended Citation}

Singh, Gurjashan, "Health Monitoring of Round Objects using Multiple Structural Health Monitoring Techniques" (2010). FIU Electronic Theses and Dissertations. 330.

https://digitalcommons.fiu.edu/etd/330 


\section{FLORIDA INTERNATIONAL UNIVERSITY}

Miami, Florida

\section{HEALTH MONITORING OF ROUND OBJECTS USING MULTIPLE STRUCTURAL HEALTH MONITORING TECHNIQUES}

A thesis submitted in partial fulfillment of the requirements for the degree of MASTER OF SCIENCE in

MECHANICAL ENGINEERING

by

Gurjashan Singh

2010 
To: Dean Amir Mirmiran

College of Engineering and Computing

This thesis, written byGurjashan Singh, and entitled Health Monitoring of Round Objects using Multiple Structural Health Monitoring Techniques, having been approved in respect to style and intellectual content, is referred to you for judgment.

We have read this thesis and recommend that it be approved.

$\begin{array}{r}\text { Igor Tsukanov } \\ \hline \text { SabriTosunoglu } \\ \hline \text { Ibrahim Tansel, Major Professor }\end{array}$

Date of Defense: November 10, 2010

The thesis of Gurjashan Singh is approved.

Dean Amir Mirmiran
College of Engineering and Computing

Florida International University, 2010 


\section{DEDICATION}

I would like to dedicate this thesis to my mother and father, BalbirKaur and Resham Singh in appreciation for their unconditional love, support, and encouragement throughout mygraduate study. 


\section{ACKNOWLEDGMENTS}

I would like to take this opportunity to thank many people who have helped and supported me towards the completion of my Master's degree. First and foremost, I would like to express my sincere gratitude to my major professor, Dr. Ibrahim N. Tansel, for his guidance, patience, and friendship throughout my graduate study. His insights and words of encouragementhave often inspired and encouraged me to overcome all difficulties. I am deeply indebted to him for his tireless support and devotion.

I would also sincerely thank my committee members, Dr. Igor Tsukanov and Dr. SabriTosunoglufor their kindly suggestions, efforts, and contributions to this work.

I would also like to thank my colleagues, Gurjiwan Singh, SrikanthKorla, Mustafa Demetgul, Ming Li and Ramon Duran for their prodigious help and support.

I would also wish to thank my friends, Puneet Gill, Amardeep Mann, SmitPandya and SrikanthKorla for their great friendship and support that made my life in FIU wonderful.

Finally, I would give all of my gratitude to my parents, my sister, HarveenDhaliwal and my brother, Gurjiwan Singh, for their unconditional love, support, and encouragement during my difficult times since I have been far away from home. 


\section{ABSTRACT OF THE THESIS \\ HEALTH MONITORING OF ROUND OBJECTS USING MULTIPLE \\ STRUCTURAL HEALTH MONITORING TECHNIQUES}

by

Gurjashan Singh

Florida International University, 2010

Miami, Florida

Professor Ibrahim N. Tansel, Major Professor

Structural Health Monitoring (SHM) techniques are widely used in a number of Non - destructive Evaluation (NDE) applications. There is a need to develop effective techniques for SHM, so that the safety and integrity of the structures can be improved.Two most widely used SHM methods for plates and rods use either the spectrum of the impedances or monitor the propagation of lamb waves. Piezoelectric wafer - active sensors (PWAS) were used for excitation and sensing. In this study, surface response to excitation (SuRE) and Lamb wave propagation was monitored to estimate the integrity of the round objects including the pipes, tubes and cutting tools.SuRE obtained the frequency response by applying sweep sine wave to surface.The envelope of the received signal was used to detect the arrival of lamb waves to the sensor. Both approaches detect the structural defects of the pipes and tubes and the wear of the cutting tool. 


\section{TABLE OF CONTENTS}

CHAPTER

PAGE

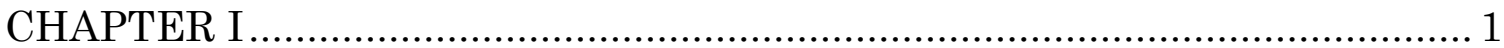

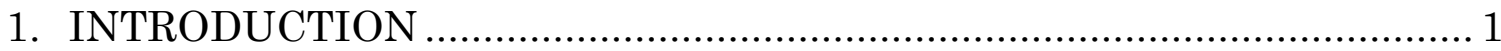

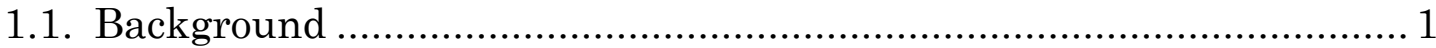

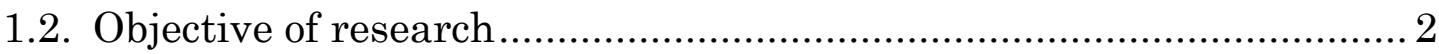

1.3. Piezoelectric Wafer Active Sensors (PWAS) ..................................... 2

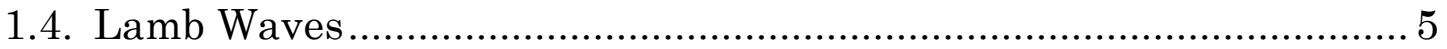

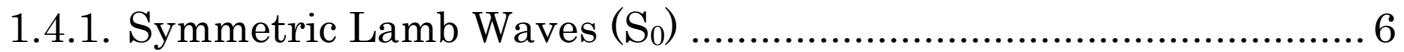

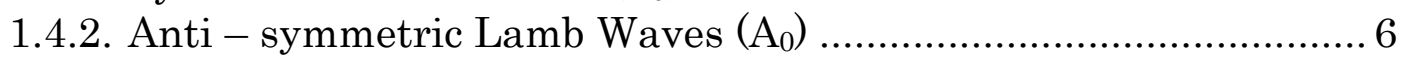

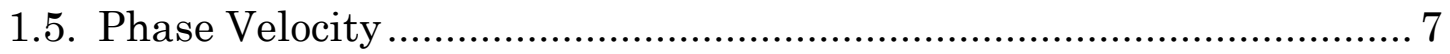

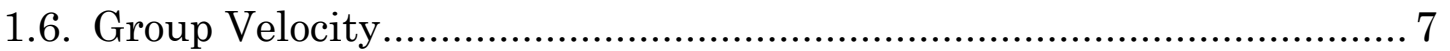

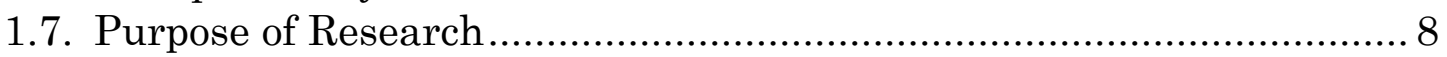

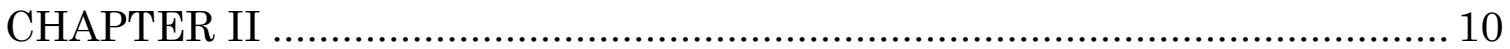

2. HEALTH MONITORING OF COPPER AND CARBON TUBES ............. 10

2.1. Guided waves in hollow shells...................................................... 10

2.2. Experimental Setup ..................................................................... 12

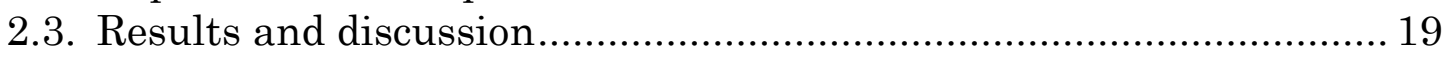

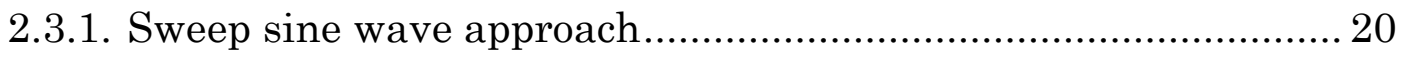

2.3.1.1. Copper pipe with cut at the center....................................... 20

2.3.1.2. Copper pipe with hole at the center ...................................... 25

2.3.1.3. Copper pipe with dent at the center ..................................... 30

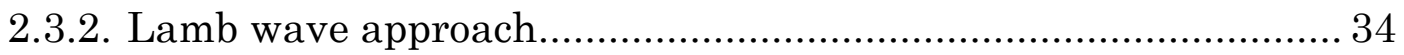

2.3.2.1. Copper pipe with cut at the center........................................ 34

2.3.2.2. Copper pipe with hole at the center ....................................... 50

2.3.2.3. Copper pipe with dent at the center ..................................... 57

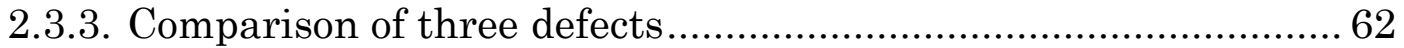

2.3.4. Determine attenuation of signal over long distance .....................63 63

2.3.5. Health monitoring of Composite Tubes against defects ...............66 66

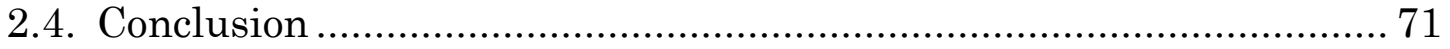

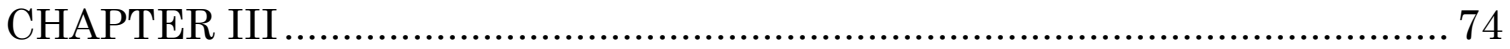

3. ESTIMATION OF DRILL WEAR THROUGH SURFACE WAVES ......... 74

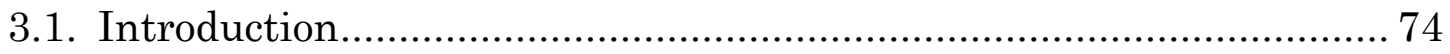

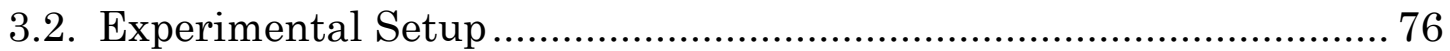

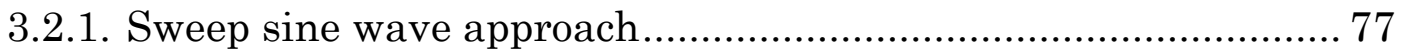

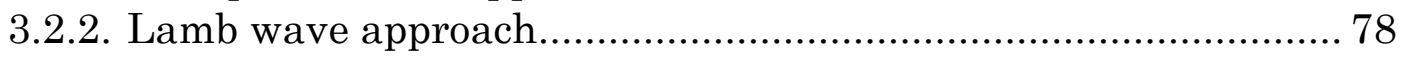

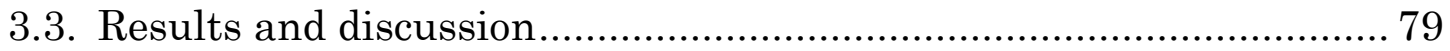

3.3.1. Surface response to excitation by sweep sine wave ..................... 79

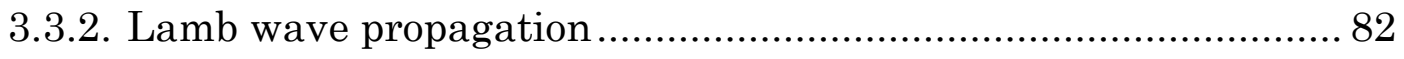




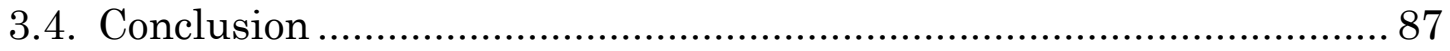

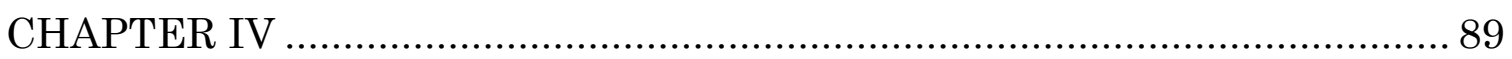

4. CONCLUSION AND FUTURE WORK ............................................ 89

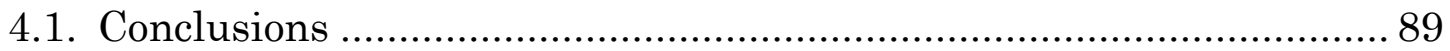

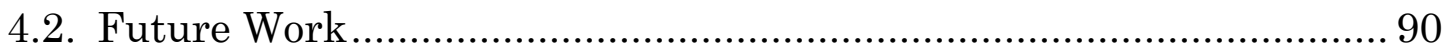

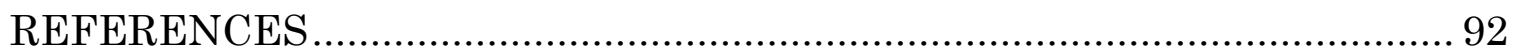




\section{LIST OF FIGURES}

FIGURE

PAGE

Figure 2.1 - Perfect copper pipe showing two piezoelectric elements (12inch in length)

Figure 2.2 - Perfect copper pipe showing two piezoelectric elements at 1 inch distance from both the sides.

Figure 2.3 - Copper pipe (a) perfect pipe, first cut and through cut (b) closer look of through cut

Figure 2.4 - Copper pipe specimen showing perfect pipe, with small hole and with large hole.

Figure 2.5 - Copper pipe specimen showing perfect pipe, with small dent and with large dent.

Figure 2.6 - Three carbon tubes (perfect, notch at center and hole at center)

Figure 2.7 - Experimental setup for sweep sine wave method...................... 18

Figure 2.8 - Lamb wave approach experimental setup.

Figure 2.9 - Spectrum analysis of perfect pipe, pipe with cut 1 and cut 2.

Figure 2.10 - Square of difference between perfect pipe and pipes with cut 1 and cut 2 ......

Figure 2.11 - Sum of square of difference between each case to the perfect pipe; perfect pipe/reference data (1), small cut (2) and through cut (3)

Figure 2.12 - Sum of square of difference of the defected pipes at four different frequency ranges

Figure 2.13 - Test case v/s sum of square of difference at four frequency intervals....

Figure 2.14 - Spectrum analysis perfect pipe, pipe with small and large hole. 
Figure 2.15 - Square of difference between perfect pipe and pipe with small and large hole.

Figure 2.16 - Sum of square of difference between perfect pipe and each test case; perfect pipe/reference data (1), small hole (2) and large hole (3)

Figure 2.17 - Sum of square of difference of each test case at four different frequency levels.

Figure 2.18 - Test case v/s sum of square of difference between each test case and baseline at four frequency intervals.

Figure 2.19 - Spectrum analysis perfect pipe, pipe with small and large dent.

Figure 2.20 - Square of difference between perfect pipe and pipe with small and large dent

Figure 2.21 - Sum of square of difference of each test case; perfect pipe/reference data (1), small dent (2) and large dent (3)

Figure 2.22 - Test case v/s sum of square of difference between each test case and baseline at four frequency intervals....

Figure 2.23 - Variation of the envelope signal with simulated pipe with defect when the $200 \mathrm{KHz} 3.5$ sine wave is applied. The perfect pipe (Data 1), pipe with first cut (Data 2) and second cut (Data 3)

Figure 2.24 - Variation of the envelope signal with simulated pipe with defect when the $300 \mathrm{KHz} 3.5$ sine wave is applied. The perfect pipe (Data 1), pipe with first cut (Data 2) and second cut (Data 3).

Figure 2.25 - The received signal at sensing piezoelectric element. 36

Figure 2.26 - S-transformation plots below $450 \mathrm{KHz}$ frequency (a) perfect pipe, (b) pipe with first center cut and (c) pipe with through center cut

Figure 2.27 - Envelope of the S-transformation of the signal. Perfect pipe (data 1), pipe with first center cut (data 2) and through center cut (data 3$)$. 
Figure 2.28 - Envelope of the original signal from perfect pipe 41

Figure 2.29 - Wavelet transformation of the original signal, its first level approximation and average error.

Figure 2.30 - Wavelet transformation of the original signal, its second level approximation and average error.

Figure 2.31 - Wavelet transformation of the original signal, its third level approximation and average error.

Figure 2.32 - Wavelet transformation of the original signal, its fourth level approximation and average error.

Figure 2.33 - Envelope of the original signal from the first center cut.

Figure 2.34 - Envelope of the original signal from the second cut.

Figure 2.35 - Wavelet transformation of the original signal from first cut, its second level approximation and average error.

Figure 2.36 - Wavelet transformation of the original signal from second cut, its second level approximation and average error.

Figure 2.37 - Absolute difference of perfect pipe (data 1) and pipe with first cut (data 2)

Figure 2.38 - Absolute difference of perfect pipe (data 1) and pipe with second cut (data 3 )

Figure 2.39 - Absolute difference of pipe with first cut (data 2) and pipe with second cut (data 3 ).

Figure 2.40 - Sum of square of difference of each test case; perfect pipe/reference data (1), small cut (2) and through cut (3) .................... 50

Figure 2.41 - The received signal at sensing piezoelectric element.............. 51

Figure 2.42 - S-transformation plots below $450 \mathrm{KHz}$ frequency (a) perfect pipe, (b) pipe with small hole and (c) pipe with large hole........ 52

Figure 2.43 - Envelope of the S-transformation of the signal. Perfect pipe (data 1), pipe with small hole (data 2) and large hole (data 3)..... 53 
Figure 2.44 - Wavelet transformation of the original signal, second level approximation of the envelope and average error; from (a) perfect pipe, (b) small hole at center and (c) large hole at center.

Figure 2.45 - Absolute difference of the envelope of signal; (a) perfect pipe (data 1) and pipe with small hole(data 2), (b) perfect pipe(data 1) and pipe with large hole(data 3) and (c) pipe with small hole(data 2) and pipe with large hole(data 2).

Figure 2.46 - Sum of square of difference of each test case; perfect pipe/reference data(1), small hole(2) and large hole(3) ........................ 57

Figure 2.47 - The received signal at sensing piezoelectric element.............. 58

Figure 2.48 - S-transformation plot, zoomed below $450 \mathrm{KHz}$ frequency

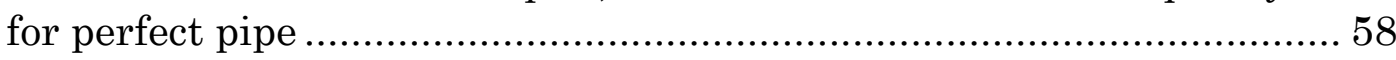

Figure 2.49 - Envelope of the S-transformation of the signal. Perfect pipe (data 1), pipe with small dent (data 2) and large dent(data 3)..... 59

Figure 2.50 - Sum of square of difference of each test case; perfect pipe/reference data (1), small dent (2) and large dent (3) ..................... 60

Figure 2.51 - Wavelet transformation of the original signal from perfect pipe, its second level approximation and average error.

Figure 2.52 - Wavelet transformation of the original signal from small dent, its second level approximation and average error.

Figure 2.53 - Wavelet transformation of the original signal from large dent, its second level approximation and average error.

Figure 2.54 - Sum of square of difference of each test case and perfect pipe/reference data

Figure 2.55 - Experimental setup for determining the attenuation of signal over long distance

Figure 2.56 - Showing strength of signal at exciter (data1), middle sensor (data2) and sensor at the end of long copper pipe (data3). 66

Figure 2.57 - Spectrum analysis of perfect tube, tube with notch and through hole. 
Figure 2.58 - Square of difference between perfect carbon tube and tubes with notch and center hole.

Figure 2.59 - The received signal at sensing piezoelectric element.

Figure 2.60 - S-transformation plot, zoomed below $600 \mathrm{KHz}$ frequency for perfect pipe

Figure 2.61 - Envelope of the S-transformation of the signal. Perfect tube (data 1), tube with notch (data 2 ) and tube with hole (data 3 )...... 70

Figure 2.62 - Sum of square of difference of each test case and perfect pipe/reference data

Figure 3.1 - Frequency response analysis experimental setup and specimen (a) experimental setup for frequency response analysis of drill-bit, (b) drill-bit equipped with Intelli-Connector MD7b data acquisition unit and (c) location of PWAS transducers on the specimen

Figure 3.2 - High speed steel drill specimen; (a) new drill and (b) worn out drill.

Figure 3.3 - Spectrum analyses of perfect and grinded tool at 4 different wear levels

Figure 3.4 - The sum of the squares of the differences between the magnitude of each case and the reference. The first and the second cases are the perfect tool. The 3,4 and 5 shows the cases for low, medium and significant wear.

Figure 3.5 - The difference between the each test case and the perfect one at different frequency intervals. The frequency intervals designated 1 to 4 corresponds to $0 \mathrm{~Hz}$ to $25 \mathrm{KHz}, 25 \mathrm{KHz}$ to 50 $\mathrm{KHz}, 50 \mathrm{KHz}$ to $75 \mathrm{KHz}$, and $75 \mathrm{KHz}$ to $100 \mathrm{KHz}$ respectively

Figure 3.6 - The received signals by the sensing piezoelectric element.

Figure 3.7 - The envelopes of the received signals by the sensing piezoelectric elements. The data numbered 7 and 8,9 and 10, and 11 and 12 were obtained at the identical simulated wear levels, and they are almost thesame. The first two belong to perfect 
(data 7,8 ), the following two corresponds to medium, and severe tool wear (data 11, 12).

Figure 3.8 - Variation of the envelope signal with simulated tool wear when the $200 \mathrm{KHz} 3.5$ sine wave is applied. The perfect (Data 1), medium (Data 3) and severe (Data 5) simulated wear.

Figure 3.9 - Variation of the envelope signal with simulated tool wear when the $300 \mathrm{KHz} 3.5$ sine wave is applied. The perfect (Data 7), medium (Data 9) and severe (Data 11) simulated wear.

Figure 3.10 - The sum of the square of the difference between each set and the first one. The excitation signal frequency was $200 \mathrm{KHz}$. The data sets of 1 to 6 were tested. The intervals $1,2,3$, and 4 corresponds to the 0-75 $\mu \mathrm{sec}, 75-150 \mu \mathrm{sec}, 150-225 \mu \mathrm{sec}$, and 225$300 \mu$ sec respectively.

Figure 3.11 - The sum of the square of the difference between each set and the first one. The excitation signal frequency was $300 \mathrm{KHz}$. The data sets of 7 to 12 were tested. The intervals $1,2,3$, and 4 corresponds to the 0-75 $\mu \mathrm{sec}, 75-150 \mu \mathrm{sec}, 150-225 \mu \mathrm{sec}$, and 225$300 \mu$ sec respectively. 


\section{CHAPTER I}

\section{INTRODUCTION}

\subsection{Background}

Structural Health Monitoring (SHM) is a technology to collect data about critical structural components using embedded sensing elements to provide detection and diagnosis when damage occurs.Common forms of structural damage include holes, cracks, notches, corrosion, etc. SHM is also a useful tool for improving the safety and reliability of the structures and to thereby reduce their operational cost.This is an emerging research area with multiple applications. There is a large number of non-destructive evaluation (NDE), non-destructive testing (NDT), and non-destructive inspection (NDI) techniques for identifying local damage and detect failure in critical structures. The most common structures being monitored by these techniques are plates, plate like structures and pipes[31]. During the last two decades, increasing resources have been put into the development of built-in damage detection systems[32]. SHM sets out to determine the health of a structure by reading an array of sensors that are embedded (permanently attached) into the structure and monitored over time. And through appropriate data processing and interpretation we may predict the remaining life of the structure in a long run. A promising damage detection method is the active system based on the propagation of Lamb Waves or guided waves [33]. 


\subsection{Objective of research}

The purpose of this study is to develop an efficient, low - cost and flexible health monitoring system. The sweep sine wave approach and lamb wave approach was implemented for this purpose. At first look the results obtained from these SHM methods were not very conclusive, so to extract meaningful information from the measured data these methods were enhanced by further processing the data through statistical methods. Dynamic frequency responses were collected by piezoelectric elements, envelope of the original signal and $\mathrm{S}$ - transformation were applied to extract the characteristics of the frequency response. Sum of square of difference for perfect and present defect was calculated to verify the intensity of defects within the system.

\subsection{Piezoelectric Wafer Active Sensors (PWAS)}

Piezoelectric wafer Active Sensors (PWAS) are non-invasive, inexpensive transducers having wideband non - resonant properties that operate on the piezoelectric principal. Since 1990 the PWAS transducers were being used by many researchers. Initially, PWAS were used for vibrations control by Crawley and Fuller. Banks et al. in 1996 used PWAS for damage detection of a structure. The use of PWAS for structural health monitoring has following 3 main paths, modal analysis and transfer function, electromechanical E/M impedance, wave propagation [26]. The use of PWAS for damage detection with lamb wave propagation was pioneered by Chang 
and his coworkers. They have studied the use of PWAS for generation and reception of elastic waves in composite materials. Passive reception of elastic waves was used for impact detection. Pitch - catch transmission - reception of low frequency lamb waves was used for damage detection

PWAS couple the electrical and mechanical effects through the tensorial piezoelectric consecutive equations

$$
\begin{aligned}
& \mathrm{S}_{\mathrm{ij}}=S_{i j k l}^{E} \mathrm{~T}_{\mathrm{kl}}+\mathrm{d}_{\mathrm{kij}} \mathrm{E}_{\mathrm{k}} \\
& \mathrm{D}_{\mathrm{j}}=\mathrm{d}_{\mathrm{kk}} \mathrm{T}_{\mathrm{kl}}+\varepsilon_{j k}^{T} \mathrm{E}_{\mathrm{k}}
\end{aligned}
$$

Where, $S_{i j k l}^{E}$ is the mechanical compliance of the material measured at zero electric field $(\mathrm{E}=0), \varepsilon_{j k}^{T}$ is the dielectric permittivity measured at zero mechanical stress $(T=0)$ and $d_{k i j}$ represents the piezoelectric coupling effect.

PWAS transducers can be used for multimode structural health monitoring by simply changing is their driving frequencies. These sensors have the potential to work and detect changes (changes can be due to any damage) in the high frequency because they are tuned for high frequency explorations. So, these sensors have an advantage over other conventional sensors (accelerometers, strain gauges etc.) working on low frequencies which encounter bandwidth problems at high frequencies [6]. In certain cases these sensors can be the only ones to be used for investigation purposes, as in the case of small turbo machinery parts, computer industry components and precision machinery etc. PWAS transducers exhibit stable and repeatable characteristics, not only in as received condition but also while working with 
1-D and 2-D structures. Moreover being very light weight, these sensors can be permanently embedded to the structure being monitored against any kind of damage [3]. The bonding material used to permanently attach the sensors has a great significance in monitoring the structural health because the signals will change due to varying operational and environmental conditions of the structure such as temperature, humidity etc [7]. So, bonding layer between the sensor and the structure is often the durability weak link for structural health monitoring applications, leading to loss of contact with the surface.You can find different types and sizes of PWAS transducers in the market but the most commonly uses among those are square and circular ones. From these two, circular PWAS transducers are mostly used because they excite omnidirectional guided waves that propagates in circular wave fronts whereas square piezoelectric elements also generate omnidirectional waves but with irregular patterns to those of circular ones [6].These sensors follow the structural dynamics and being non - resonant wideband devices, can achieve in - plane lamb wave excitation and sensing through in-plane strains [4]. Embedded piezoelectric wafer active sensors can be used as both individual probes and phased arrays.

The use of PWAS for SHM has several main paths including highbandwidth strain sensors, PWAS converts the mechanical energy into electrical energy and the conversion factor obtained is linearly dependent on the signal frequency. This is the reason that we can directly connect the 
PWAS transducers to high impedance measuring instruments (digital oscilloscope). PWAS can also be used as high bandwidth strain exciters. In this way PWAS can easily induce vibrations and waves in the structure to which it is attached.

This Embedded Non-Destructive Evaluation (NDE) with PWAS transducers opens new ways of performing in - planedamage detection and structural health monitoring of thin walled structures such as pipelines, pressure vessels, oil tanks, missiles and aircrafts.

\subsection{Lamb Waves}

The theory of lamb waves (also referred as plate waves or guided waves) was originally developed by Horace Lamb in 1916 to describe the characteristics of waves in plates. Guided wave (e.g. Lamb Waves) technique is one of the best method for thin walled structures (sheet materials, tubular structures). Extensive developments in the application of lamb waves provide a platform for the inspection of many industrial products in aerospace [24], pipe and transportation industries. The generation of lamb waves can be performed using contact transducers, optical electromagnetic, magnetostrictive and air coupled transducers [34]. These waves can travel a long distance inside the thin walled structures at a very little cost of amplitude loss, making it possible to detect flaws over a considerable area $[29,30]$. Lamb wave transmission and reception can be easily performed with the PWAS transducers as they propagate through the mid-surface of the thin 
walled structure and can sweep large surface area with minimum installed sensors [4]. Complications that are encountered include the existence of multiple modes and their dispersive behavior. The solution to this complex problem can be achieved by using the transducers which excite only a single mode [35]. Generally conventional transducers are used to generate guided waves but they are very expensive and their bulkiness makes them nonfeasible for structural health monitoring. The two modes of Lamb waves are $\mathrm{S}_{0}$ and $\mathrm{A}_{0}$ mode

\subsubsection{Symmetric Lamb Waves $\left(\mathrm{S}_{0}\right)$}

In the low frequency regime, the zero order symmetrical mode travels at the plate velocity (considering plate as specimen), where it is also known as the extensional mode or longitudinal mode. During this period the plate stretches in the direction of propagation of wave and correspondingly contracts in the thickness direction. At increased frequency the wavelength of the wave becomes comparable to the plate thickness, now at this stage the plate starts to have a significant influence on its effective stiffness. The phase velocity drops smoothly while the group velocity drops abruptly towards a minimum.

\subsubsection{Anti - symmetric Lamb Waves $\left(\mathrm{A}_{0}\right)$}

In the low frequency regime, the zero order anti-symmetric modesare highly dispersive in nature, where it is also known as flexural mode. At low frequencies, the phase and group velocities are both proportional to the 
square root of the frequency. Here the group velocity is twice the phase velocity.

Lamb waves can be generated in a plate with free boundaries with an infinite number of modes for both symmetric and anti-symmetric displacements within the layer. The average displacement over the thickness of the plate layer is in the longitudinal direction and transverse direction for symmetric and anti-symmetric modes respectively. The longitudinal mode, generally have higher velocity and low amplitude than the flexural mode but the flexural mode is the more easily excited of the two modes and often carries most of the energy.

1.5 Phase Velocity

The phase velocity of a wave is the speed for a wave of a single wavelength or you can say that it is the rate at which the phase of the wave propagates in space. This is the speed at which the phase of any one frequency component of the wave travels.

$\vartheta_{p}=\frac{\omega}{K}$ Where, $\omega=$ Angular velocity and $\mathrm{K}=$ Wave number.

\subsection{Group Velocity}

The group velocity of a wave is the velocity with which the overall envelope of the wave propagates through the space. It also defines the energy and information of the wave.

$\vartheta_{g}=\frac{\partial_{\omega}}{\partial_{k}} \quad$ Where, $\omega=$ Angular velocity and $\mathrm{K}=$ Wave number 
It has been shown experimentally and theoretically that the $\mathbf{S}_{0}$ lamb waves can give better reflections from the cracks than the $\mathrm{A}_{0}$ lamb waves [6]. 1.7 Purpose of Research

The main objective of this research is to develop an accurate and cost effective process for detection of defects in pipes, tubes and cutting tool. A system with sensors will allow for the early detection of change in characteristics and failures thereby increase the safety of the system. For nondestructive evaluation (NDE) applications, PWAS can be used as embedded ultrasonic transducers [6, 3]. Upon excitation PWAS generate guided waves that can be used for damage detection through pulse-echo, time reversal and pitch-catch techniques [14-17]. The guided waves detect the defect or change in structure depending on its interaction with the propagating elastic wave. For this purpose few sensors and actuators are required for inspecting a large area [8]. These waves can travel through large distances with very little amplitude loss. A large number of guided wave inspection systems have been used for various applications, including damage detection in composite materials [9-10], defect detection in pipes [11-12], crack detection of steel bridge components [13], corrosion detection [28], health monitoring of rotorcraft [25], etc. PWAS act as both lamb wave exciters and transmitters. These low-cost, commercially available piezoelectric ceramics has opened a number of ways for dynamic structural identification by making use of embedded active sensors. These sensors are 
small piezoelectric Transducer (PZT) ceramic wafers of negligible mass that can be easily and permanently applied to the structure creating a nonintrusive sensor array for automatic structural identification and health monitoring [3]. Based upon the above theory, we propose to detect any changes or defects in pipes, tubes and cutting tool using guided waves generated by piezoelectric wafer active sensors. 


\section{CHAPTER II}

\section{HEALTH MONITORING OF COPPER AND CARBON TUBES}

\subsection{Guided waves in hollow shells}

Guided waves in hollow cylinders (pipes, tubes etc.) were first studied by Gazis [37]. According to him the guided waves in a hollow cylinder have a double infinite number of modes and the energy is constrained inside the tube wall. These waves fall into three sub categories torsional, longitudinal and flexural [39].The longitudinal and torsional modes fall into axisymmetric mode whereas flexural mode fall into non - axisymmetric mode and are found and represented in phase and group velocity dispersion diagrams. The torsional modes induce particle motion in the circumferential direction (twisting of a pipe as they propagate), while longitudinal and flexural modes induce particle motion both along the main axis of the pipe and orthogonal to the surface of the pipe, and arise as an extension of the symmetric and antisymmetric modes present in thin plates [11]. Torsional and longitudinal modes are both axisymmetric, while flexural modes are not.The evolution of wave structures of different modes at different frequencies has also been discussed by Gazis [37]. When frequency goes very high, the higher order modes will backslide into surface waves. Surface waves are a superposition of lower order longitudinal modes at very high frequency [38]. The longitudinal mode is very effective for testing for the following reasons: it is practically 
non - dispersive over a wide bandwidth, which means its velocity does not vary significantly with frequency and this will retains the signal shape and amplitude. It is equally sensitive to internal and external defectsat any circumferential location.

Another group of researchers, Silk and Bainton (1979) showed that for a thin walled pipe, the assumption of Lamb wave propagation is valid. The longitudinal $\mathrm{L}(0,1)$ mode in the pipe is equivalent to that of $\mathrm{A}_{0} \mathrm{Lamb}$ wave mode in plate. The shell structure (e.g. large pipes) is very similar to that of the propagation of the guided waves within the structure.

A number of authors have showed the work on the use of lamb waves for the inspection op pipes and tubes [5, 56, 57]. The use of circumferential travelling waves has also been studied for the inspection of larger diameter gas pipelines [58]. Publications have also been reported the development of the dry - coupled transducers for the excitation and detection of guided waves [58].

As in United States there are about 300 thousand miles of transmission pipelines and over one million aging distribution pipelines and these needs to be monitored regularly [27]. Failure of a single pipeline (gasoline, water, etc.) can cause a huge damage.In United States alone, on an average there were approximately 80 all pipeline system excavation damage incidents in 2009 [18].Pipeline degradations are generally not monitored due to lack of any reliable and durable techniques [1]. However, to find the 
damaged areas of the underground pipelines through visual inspection is very expensive and time consuming [2]. One of the major difficulties of monitoring these pipeline networks is the fact that the length of pipeline can be few meters to hundreds of kilometers buried underground [5]. One more difficulty for monitoring the pipe against any kind of defect can be the fact that the pipe may be partially buried, or insulated. So, it may not be practical to uncover the entire length of pipe for health monitoring. Because of the NDE and non-invasive characteristics of piezoelectric transducer (PZT) active sensors, routine maintenance of pipes (either underground, insulated or above the ground) in service will then be possible by implementing Nondestructive Testing (NDT) techniques. In this chapter we will discuss SHM techniques and work on the nondestructive testing methods for the monitoring of pipes, through easily available sensors and controllers which is competent enough to allow the localization of defects along the length of the pipe and will be able to give us a rough idea about the location and size of the defect. It is therefore envisaged that defects, leaks, cracks and any other degradation effects due to aging of pipes can be pointed out by nondestructive evaluation techniques using piezoelectric wafer-active sensors.

\subsection{Experimental Setup}

The goal of the experiment in the present work is to apply the SHM methods to monitor the defects in copper tubes. It is also favorable to investigate whether it is possible to find out the location of the defect in 
copper pipe embedded with two piezoelectric sensors and also to check the working principle behind lamb wave generation and propagation.

Four copper pipes were used for performing the tests. Three copper pipes were of same dimensions (12 inch length, 0.875 inch outer diameter and 0.03 inch wall thickness) and fourth copper pipe was having 120 inch length, 0.875 inch outer diameter and 0.03 inch wall thickness and can be seen in Figure 2.1.

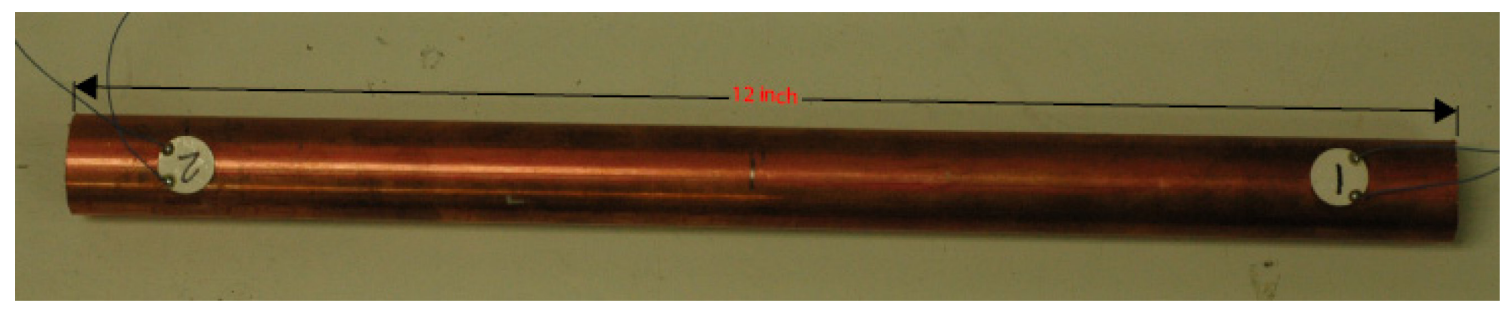

Figure 2.1 - Perfect copper pipe showing two piezoelectric elements (12 inch in length)

Each of the three copper pipes was embedded with two round piezoelectric elements of 0.50 inch diameter and 0.20 inch thickness. The piezoelectric elements were located at 1 inch from both the ends as shown in figure 2.2. One of the sensor acts as actuator (marked 1) and other as receiver (marked 2). 


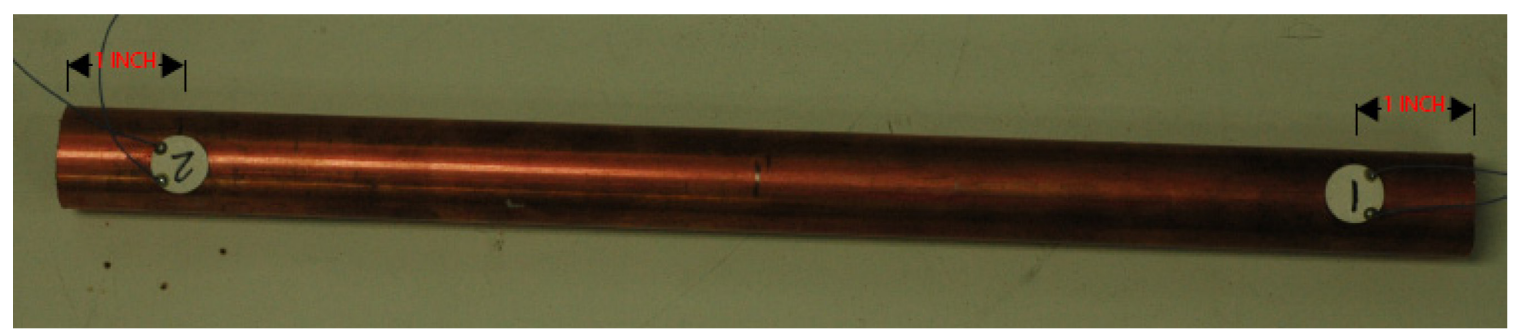

Figure 2.2 - Perfect copper pipe showing two piezoelectric elements at 1 inch distance from both the sides.

At beginning of the experiment, all the three pipes were in perfect condition and were marked as pipe 1, 2 and 3. Three different kinds of defects were created (a through cut on pipe 1, a hole on pipe 2 and a kind of dent on pipe 3) on the pipes and readings were taken at each step. Each defect was created at the center of the pipe and is shown in figure 2.3, 2.4 and 2.5. The intensity of the defect was increased after each step and in total 3 readings were taken (first of the perfect pipe, second one with less intense defect and the third one with more intense defect).

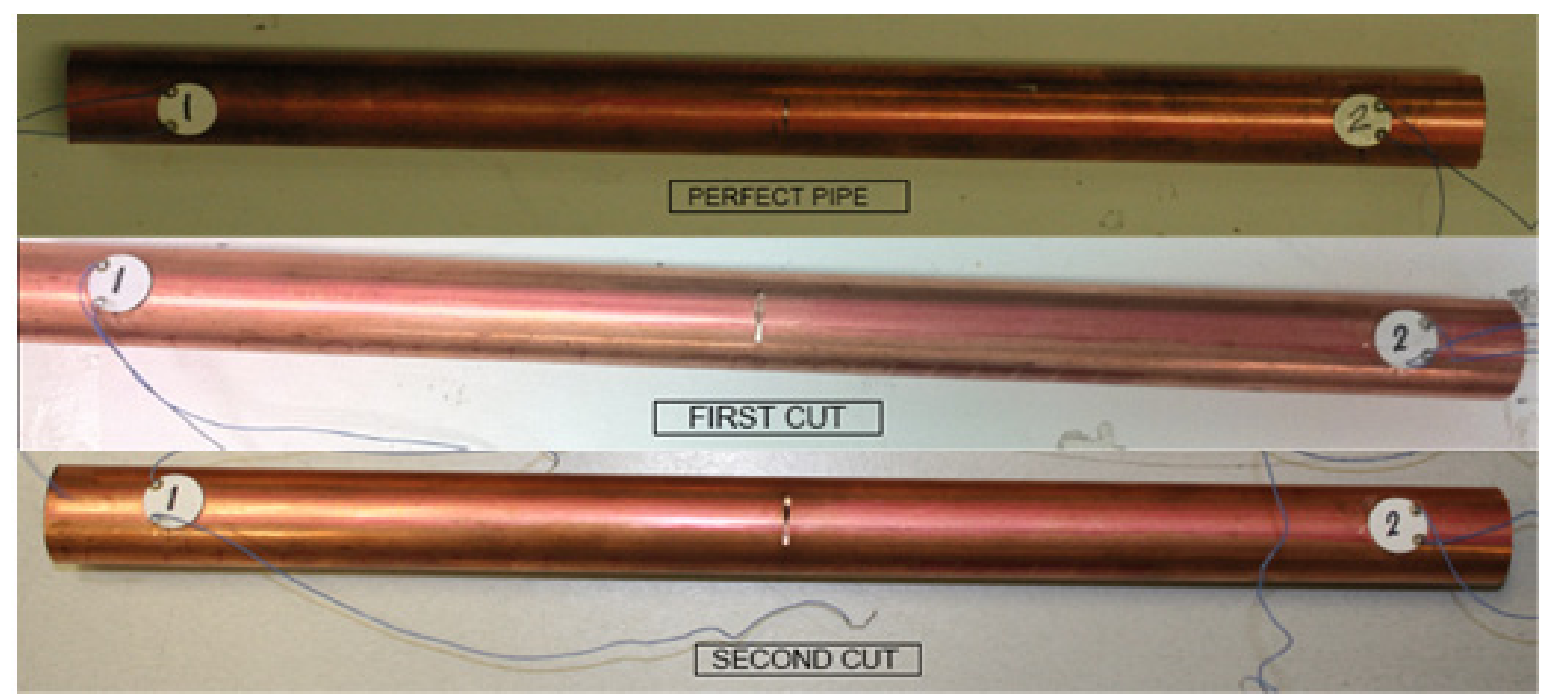

(a) 
(b)

Figure 2.3 - Copper pipe (a) perfect pipe, first cut and through cut (b) closer look of through cut

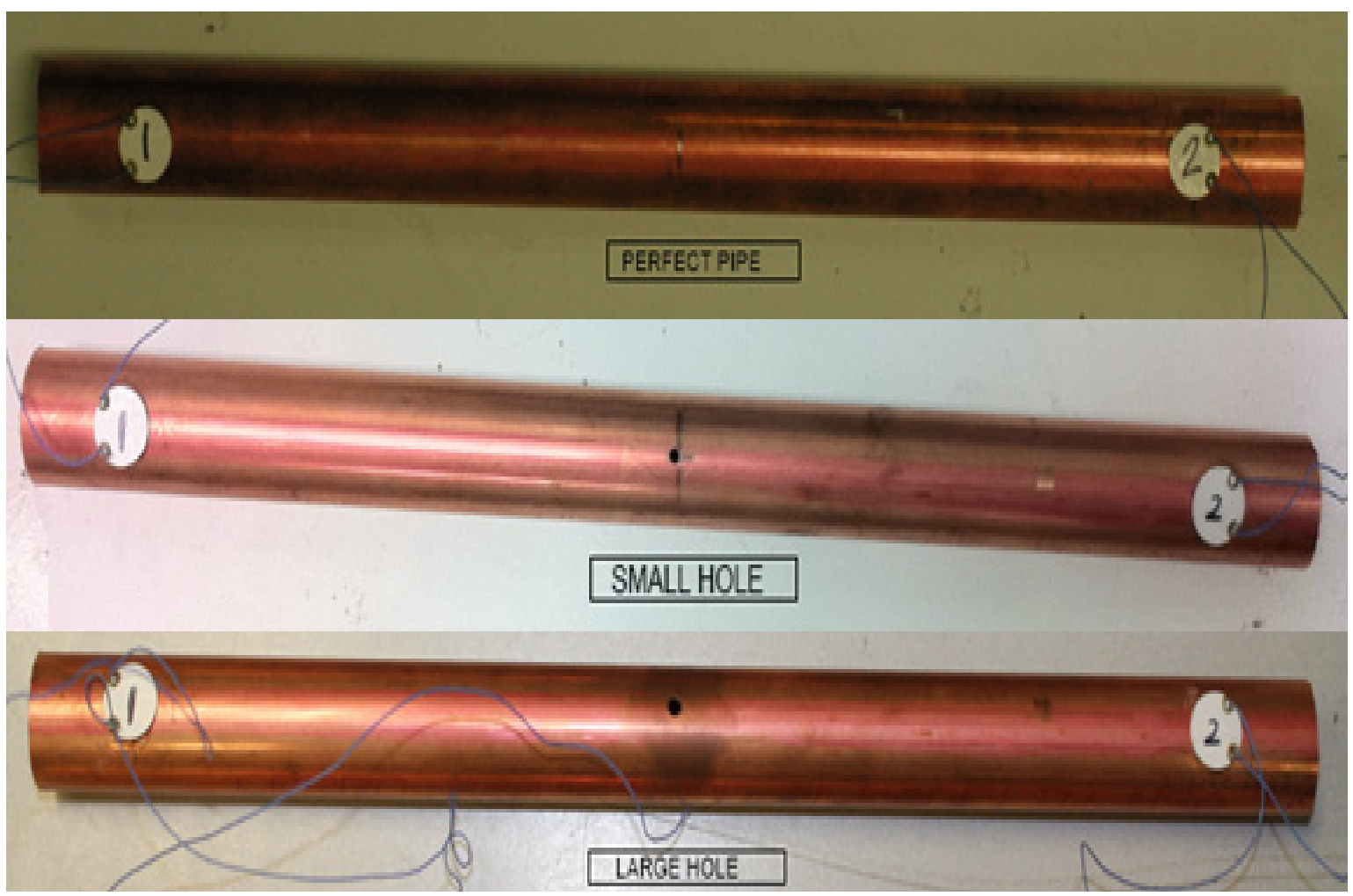

Figure 2.4-Copper pipe specimenshowing perfect pipe, with small hole and with large hole. 


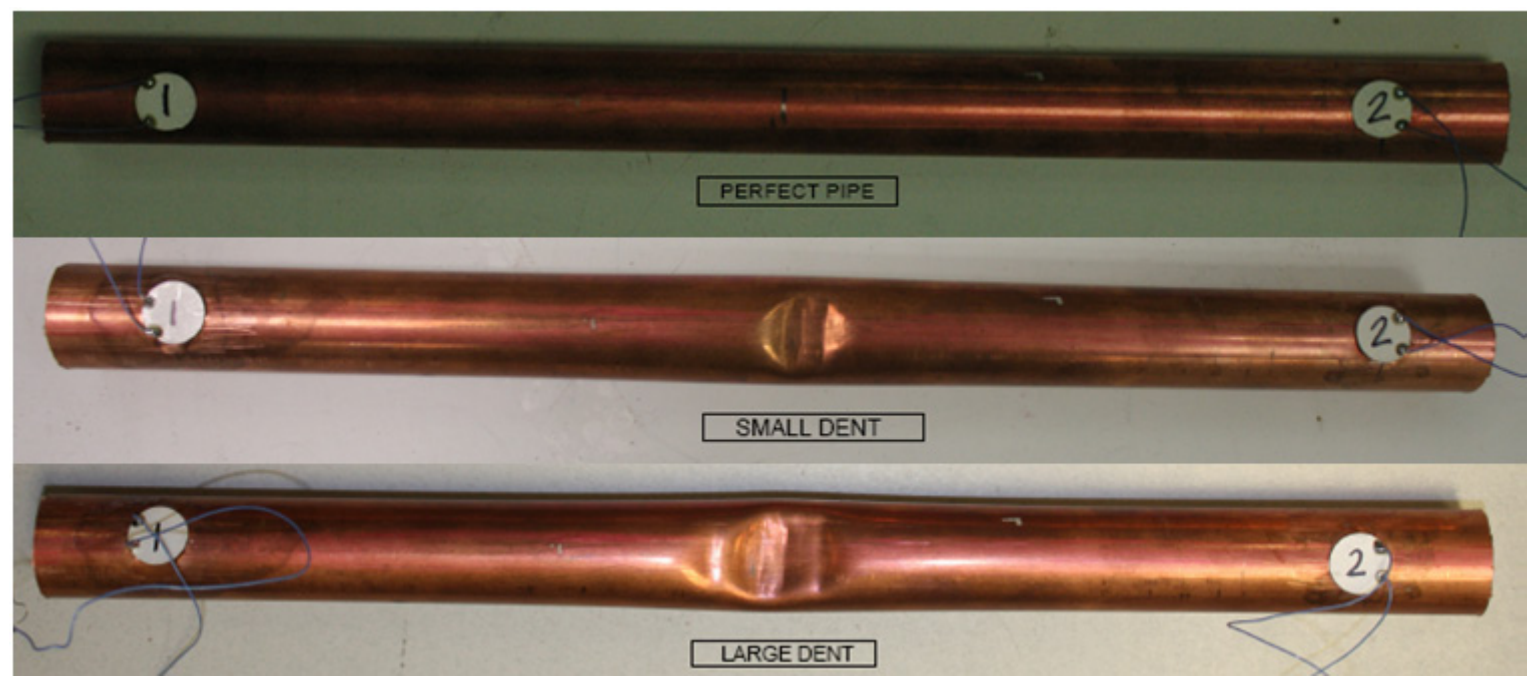

Figure 2.5 - Copper pipe specimen showing perfect pipe, with small dent and with large dent.

The data obtained from the perfect pipes was used as reference data against their respective pipes with different defects. The fourth copper pipe was just used to see the propagation of the signal i.e. how much the signal attenuates by varying the distance between the actuator and the sensor. The data was taken by using two SHM approaches: Sweep sine wave and Lamb wave approach.

Three carbon tubes of 7.5 inch length, 0.2 inch outer diameter and 0.05 inch thickness were also prepared for performing the same set of experiments. Two round piezoelectric elements of 0.25 inch outer diameter and 0.020 inch thickness was glued $(\mathrm{M}-$ Bond 200) at both the ends. One tube was kept perfect, a notch by removing approximately half or one-fourth of the material at the center and a through hole at the center was prepared to simulate the defects in the tube and can be seen in the figure 2.6. 


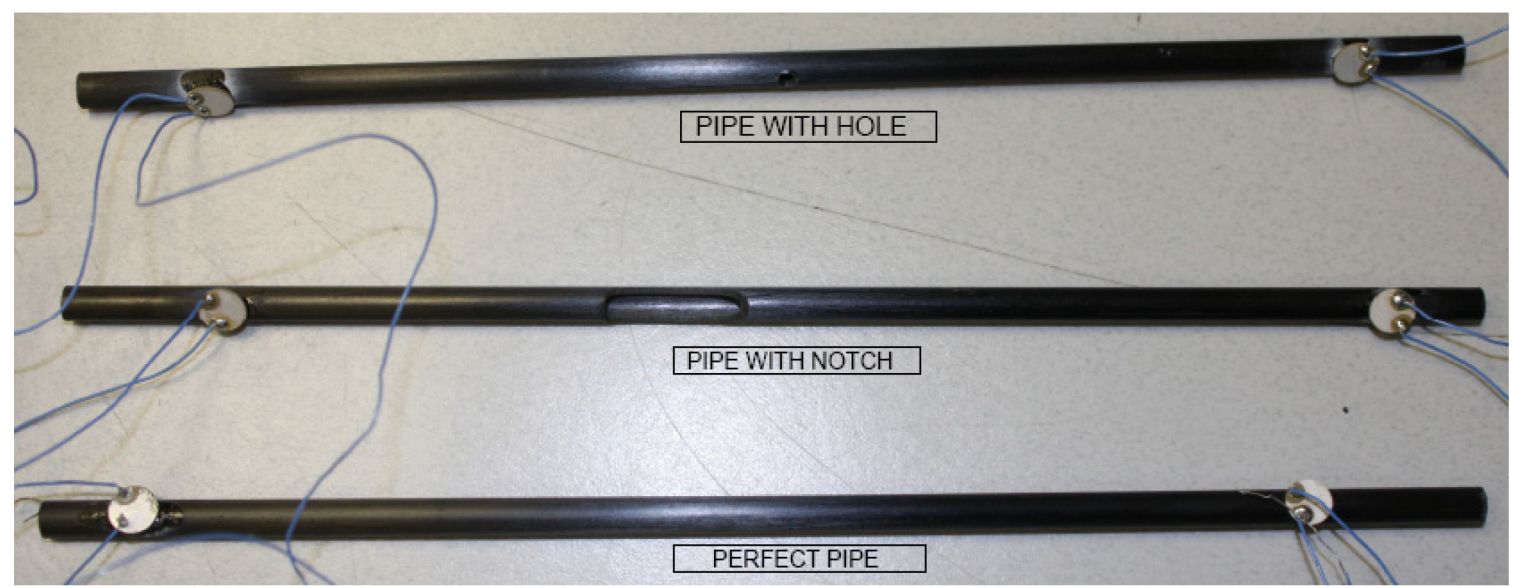

Figure 2.6 - Three carbon tubes (perfect, notch at center and hole at center)

The experimental setup to monitor the surface response by sweep sine wave approach is shown in figure 2.7. The signal analyzer (Stanford Research Systems SR780) was used for excitation as well as for the reception of signal. A sweep sine wave signal (frequency range of $0-100 \mathrm{KHz}$ ) was given to excite the actuator (sensor 1) and the signal was received (at 2000 data points) from the receiver (sensor 2) by the same equipment. The frequency domain signal was recorded for all the three equal pipes after every step. Here the magnitude of the signal received from the perfect copper pipes (pipe 1, pipe 2 and pipe 3) was taken as reference in order to compare the output signals from the same pipes for the other two stages. 


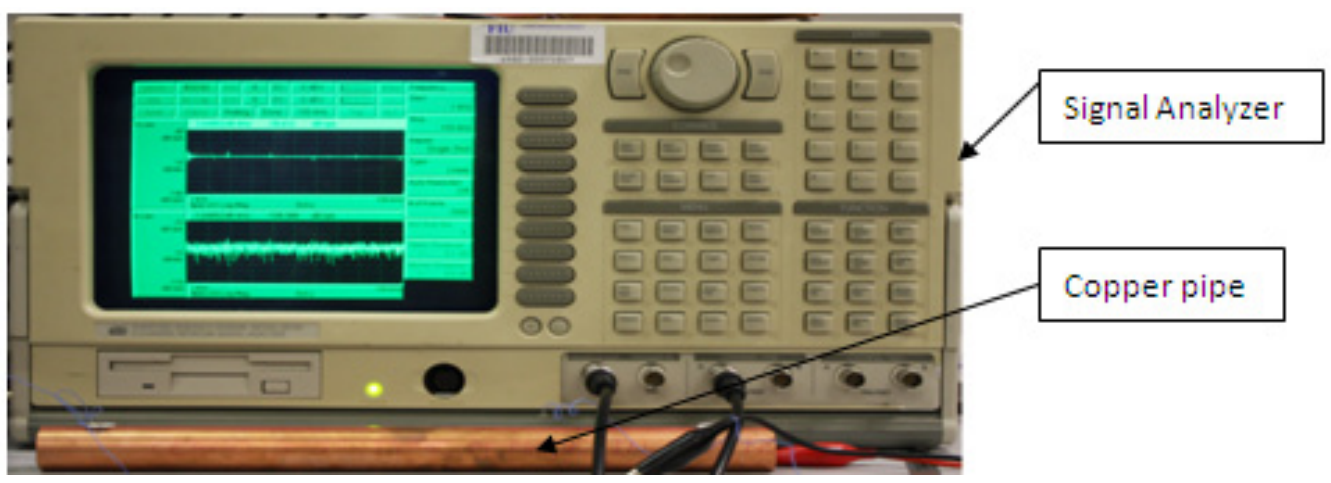

Figure 2.7 - Experimental setup for sweep sine wave method

The experimental setup for Lamb wave approach is shown in figure 2.8. To perform the experiment, Metis Design SHM Node MD 75602 was used. This equipment excites one of the piezoelectric element (marked 1) by 3.5 sine wave with $300 \mathrm{KHz}$ frequency and at the same time monitors the Lamb wave propagation through the received signal (with $10 \mathrm{MHz}$ sampling frequency, at 8000 data points and were averaged 64 times to minimize the noise contamination) from the other piezoelectric element (marked 2). From the received 8000 data points only 3000 of them were used because of the software limitation.Before giving the exciting signal to the piezoelectric element, that signal was first passed through the Hanning window because the smoother cutting of the time interval by this type of window eliminates the discontinuities associated by using other windows (rectangular window, the flat top window, etc.) and it also reduces both leakage and ripples, thus improving the selectivity of the signal. The Hanning window is the most commonly used window for continuous signals and is recommended due to the good frequency resolution associated with the window. This window is 
considered to be a general purpose window. The received signal was further processed to extract the useful information from it. $\mathrm{S}$ - transformation was used to calculate the envelopes of the received signal, wavelet transformation was then calculated of the $\mathrm{S}$ - transformation function, the significance of the differences among the envelopes was then evaluated by calculating the sum of the squares of the differences and followed by the comparison of the envelope of the two signals (perfect and with defect).

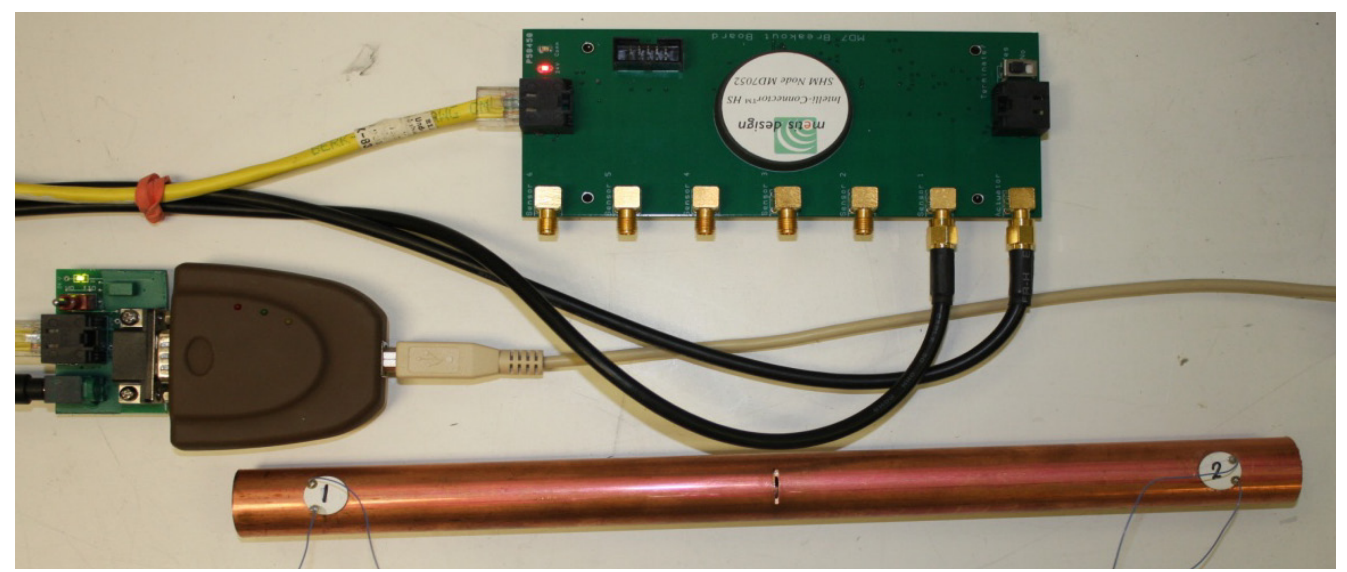

Figure 2.8 - Lamb wave approach experimental setup.

2.3Results and discussion

The above two approaches were tested for the copper pipes having different kind defects and competence, accuracy and feasibility is discussed in this section. 


\subsubsection{Sweep sine wave approach}

The experimental data gathered by testing three copper pipes with different defects on each of them was analyzed. Several plots were obtained and are discussed below. Results of pipe with cut (pipe 1) is discussed first and then followed by the pipe with hole (pipe 2) and pipe with dent (pipe 3) and lastly some experiments were done for the long copper pipe (pipe 4), in order to check the attenuation of the signal when it travels a long distance.

\subsubsection{Copper pipe with cut at the center}

The magnitude of the transfer function is calculated after each step i.e. when the pipe is perfect then after first cut (cut 1) and finally after second cut (cut 2) and can be seen in the figure 2.9. From this plot showing three windows, it is very difficult to find a difference between the three signals. So, that means just by getting the signal from the signal analyzer does not solves our problem. Some useful information has to be extracted out of the received signal by some statistical algorithm [19-21].So, for that, square of difference between the signals of cut 1 and cut2 from the perfect pipe signal can give some quantifiable information out of the measured signal which can be seen in figure 2.10. It can be seen from the figure that in the marked area, the amplitude of the signal for the plot between data 1 (perfect pipe) and data 3 (cut 2) is significantly bigger than the difference between data 1 and data 2 (cut 1) at $50 \mathrm{KHz}-60 \mathrm{KHz}$ range. This significant difference in the amplitude level is because of the difference in the intensity of the cut, as cut 2 
is much deeper than that of cut 1 . As the amplitude difference came almost at the same frequency range, therefore we can say that this could be a possible location of the defect created on the copper pipe.
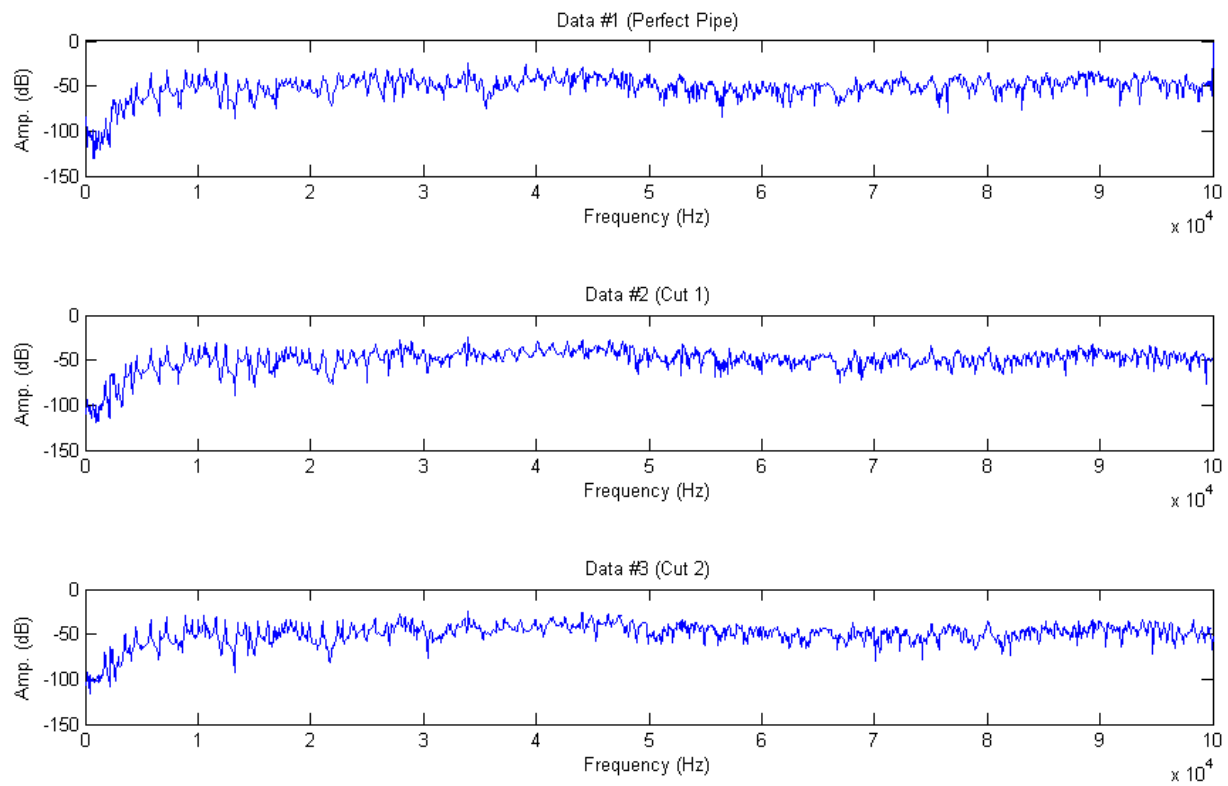

Figure 2.9 - Spectrum analysis of perfect pipe, pipe with cut 1 and cut 2 .
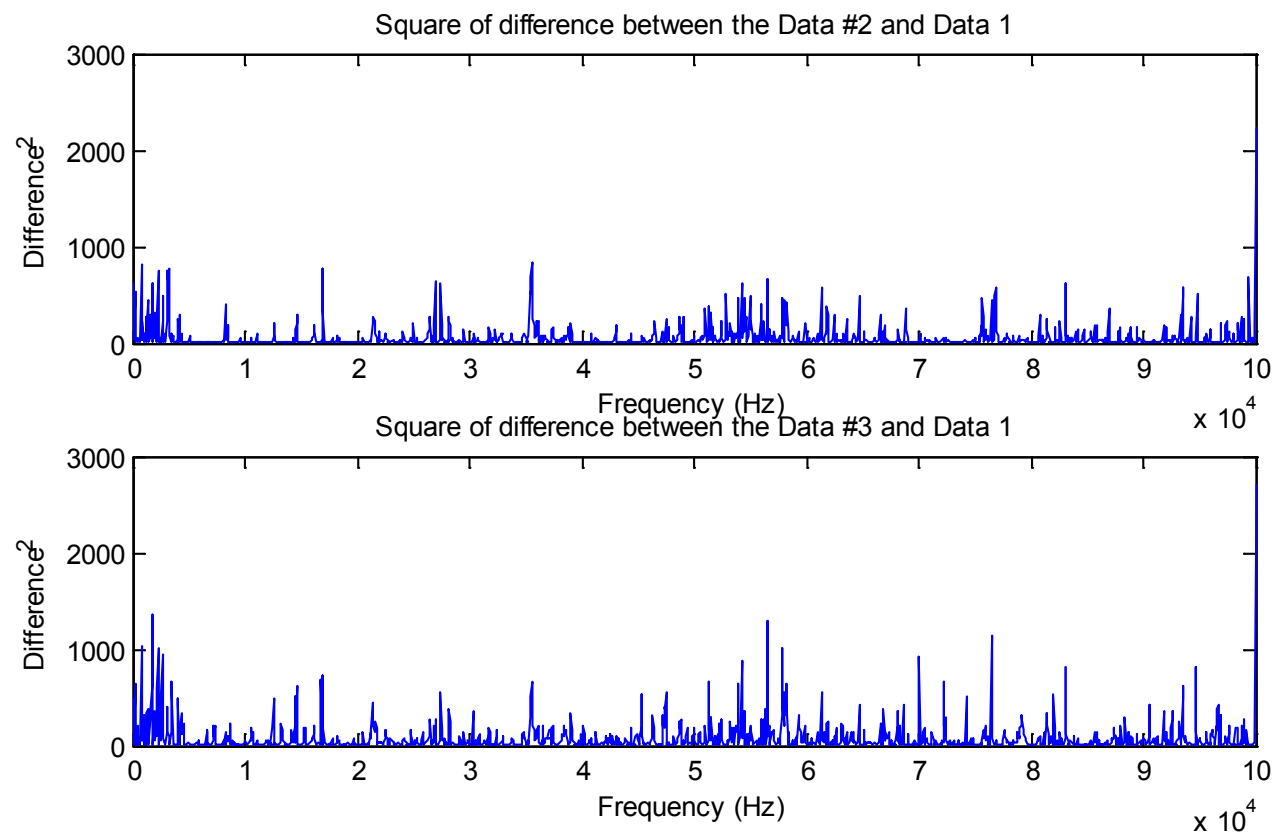

Figure 2.10 - Square of difference between perfect pipe and pipes with cut 1 and cut 2 . 
Further, in order to solidate and to make the results conclusive, sum of the squares of the difference between each case was calculatedand can be seen in figure 2.11. In this figure bar 1 represents the difference between the perfect pipe and the reference signal (signal from the perfect pipe), the bar 1 is nil because this bar represents the value for the perfect tool. The bar 2 represents the difference between the perfect pipe and the pipe with cut 1 and similarly bar 3 represents the difference between the perfect pipe and pipe with cut 2 . The length of the bar 2 from bar 1 has a significant increase than that between bar 2 and bar 3 . From this you can see the difference between the perfect pipe and the pipe with first cut is very large than that between the first cut and second cut. So, we can say that a small change (defect) in the pipe can be monitored with the help of this technique, which can be very helpful to such pipeline systems, for which the degradation monitoring is a very sensitive and essential issue. 


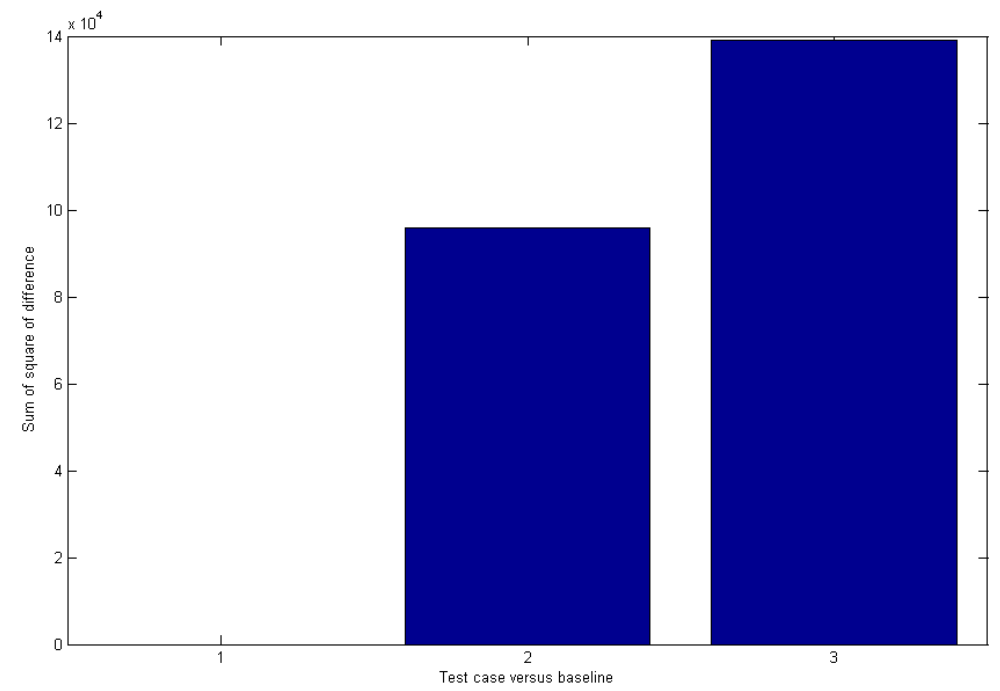

Figure 2.11 - Sum of square of difference between each case to the perfect pipe; perfect pipe/reference data (1), small cut (2) and through cut (3)

In order to see, where (frequency range) the difference between each set case is coming out to be maximum, a graph with four different frequency levels from $5 \mathrm{KHz}$ to $100 \mathrm{KHz}$ was plotted and is shown in figure 2.12. It can be clearly spotted from the figure that in the last plot the difference of sum of squares is coming out to be the maximum. So, here also the maximum difference is coming out in $50 \mathrm{KHz}$ to $100 \mathrm{KHz}$ range. 

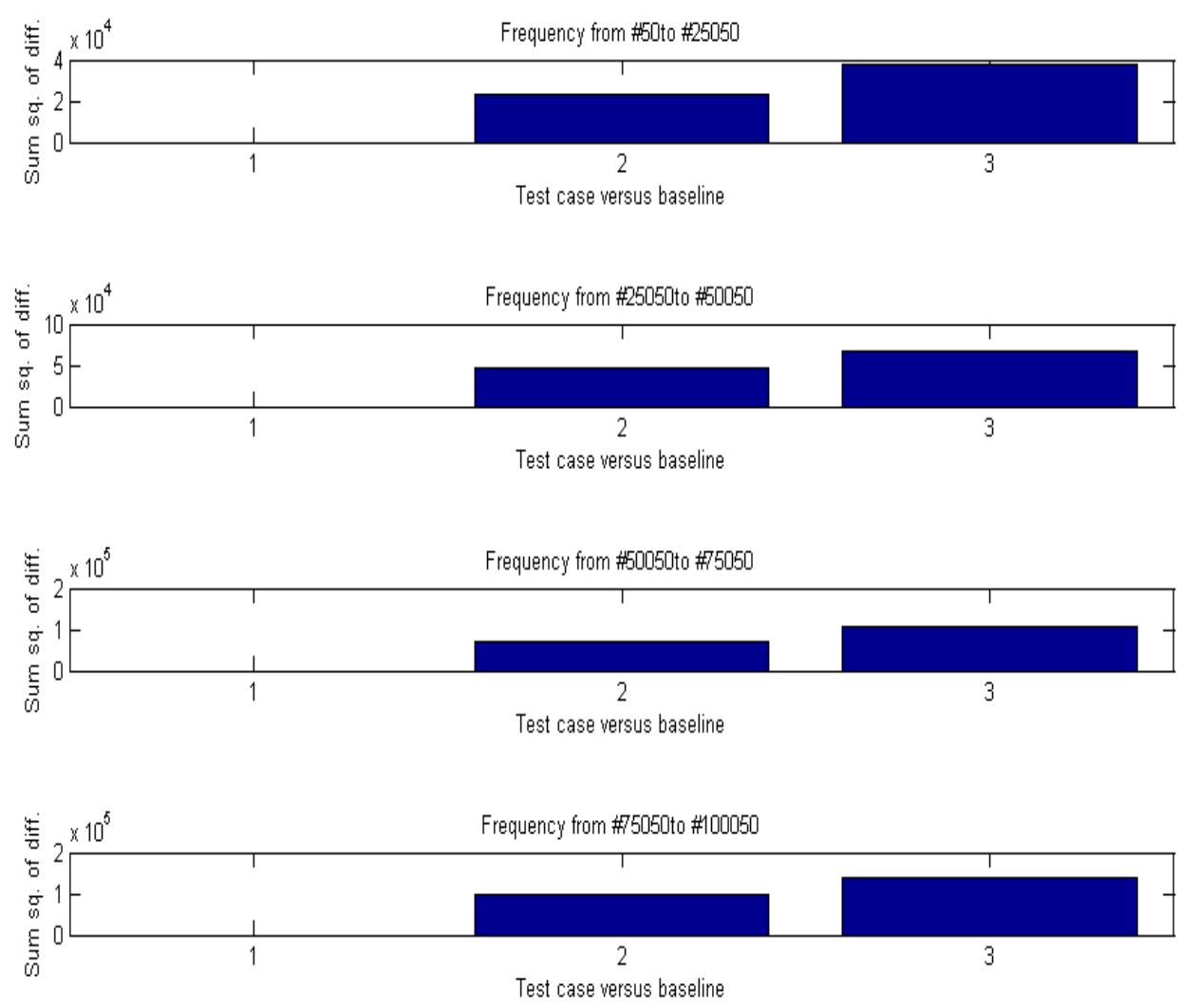

Figure 2.12-Sum of square of difference of the defected pipes at four different frequency ranges

Another way of expressing the difference of the sum of the square between each case is presented in the figure 2.13. Here you can see that for the fourth set of frequency interval $(50 \mathrm{KHz}-100 \mathrm{KHz})$ the value of difference of the sum of squares is coming out to be the maximum. 


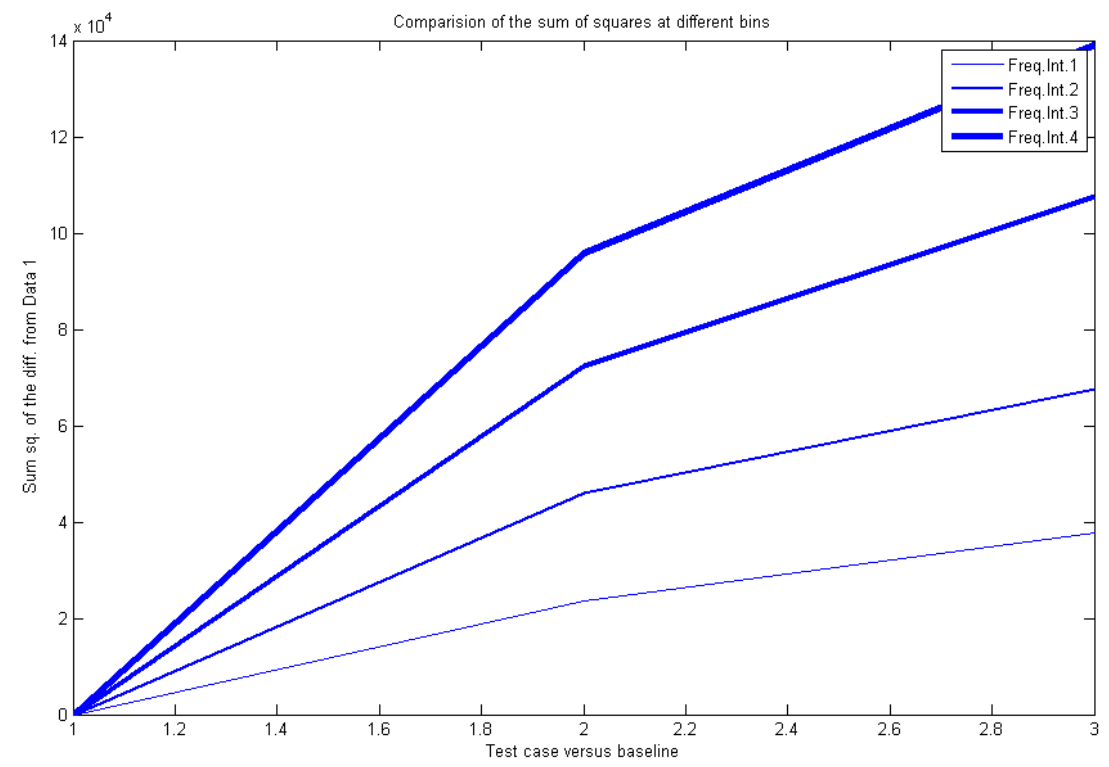

Figure 2.13 - Test case v/s sum of square of difference at four frequency intervals

\subsubsection{Copper pipe with hole at the center}

Second set of experiments were carried out on second pipe (pipe 2). For this pipe also three set of data has been taken one at each step, when the pipe was perfect, pipe with 0.090 inch diameter hole at the center and pipe with 0.117 inch diameter at the center respectively. The results for this pipe came out to be similar as those for the pipe with cut (pipe 1). The plot of magnitude function is shown in figure 2.14 . The signal came out is very rough and unclear. It is very difficult to judge anything out of this signal. All the three signals look very similar that it is almost impossible to jump on to any conclusions. The data which we got from the signal analyzer is in crude form and needs to be filtered and processed, in order to extract some meaningful information out of it. 

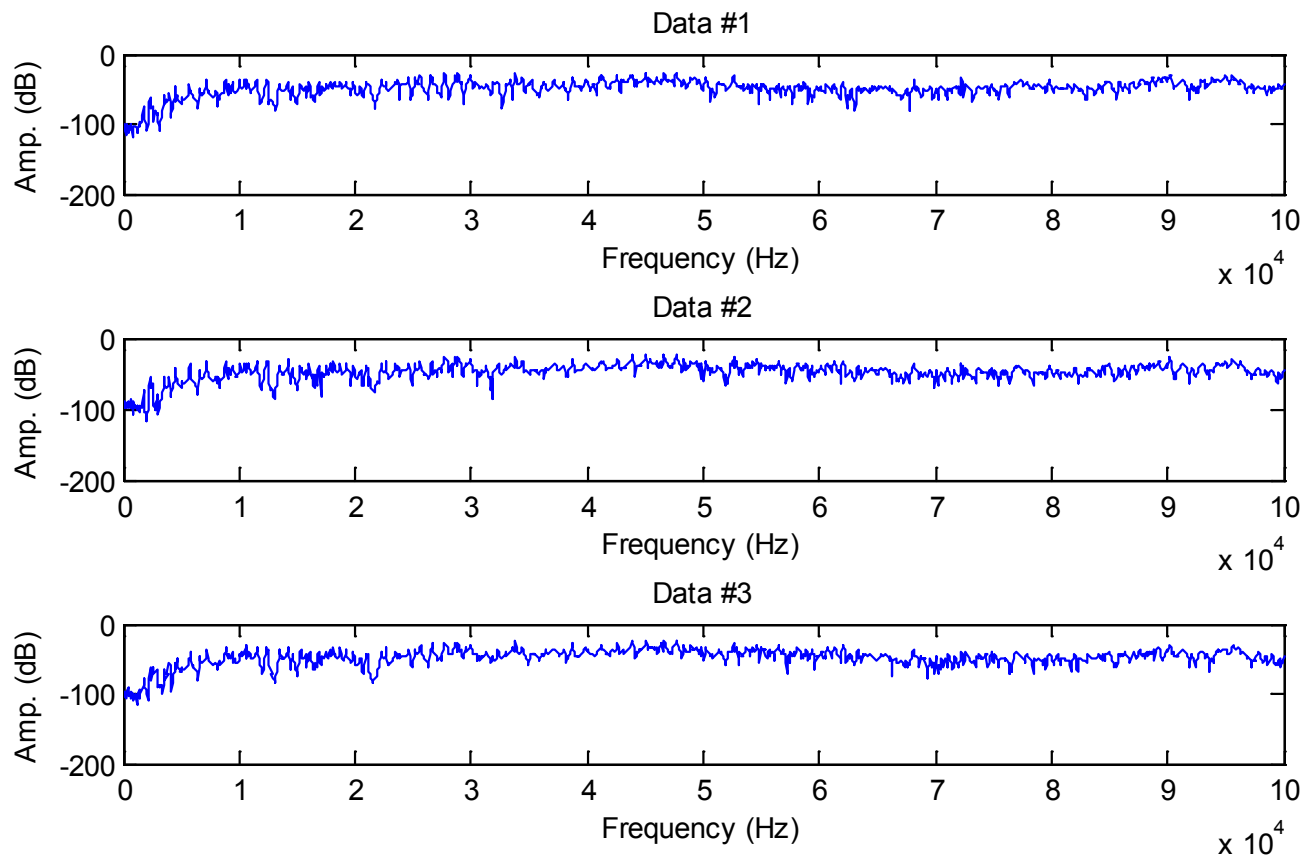

Figure 2.14 - Spectrum analysis perfect pipe, pipe with small and large hole.

To clearly find a distinctive difference among the three set of signals shown in figure 2.14, square of difference between the signals was calculated and plot of the calculated data is shown in figure 2.15. It can be seen from the figure that at around $60 \mathrm{KHz}$ the amplitude of both the signals starts changing. As for data 2 and data 1 the value of maximum amplitude is between $500-1000$ and after $60 \mathrm{KHz}$ the amplitude level starts diminishing and is always below 500 whereas for the plot between data 3 and data 1 the value of maximum amplitude at nearly $60 \mathrm{KHz}$ is almost approaching 1000 and after that also the amplitude remains somewhat near 500 as shown by dotted lines. It is therefore clear from the graphs that the intensity of the amplitude value changes (increases) with the increase in defect level 
(intensity) that means more is the severity of the defect more will be the change in the amplitude value.
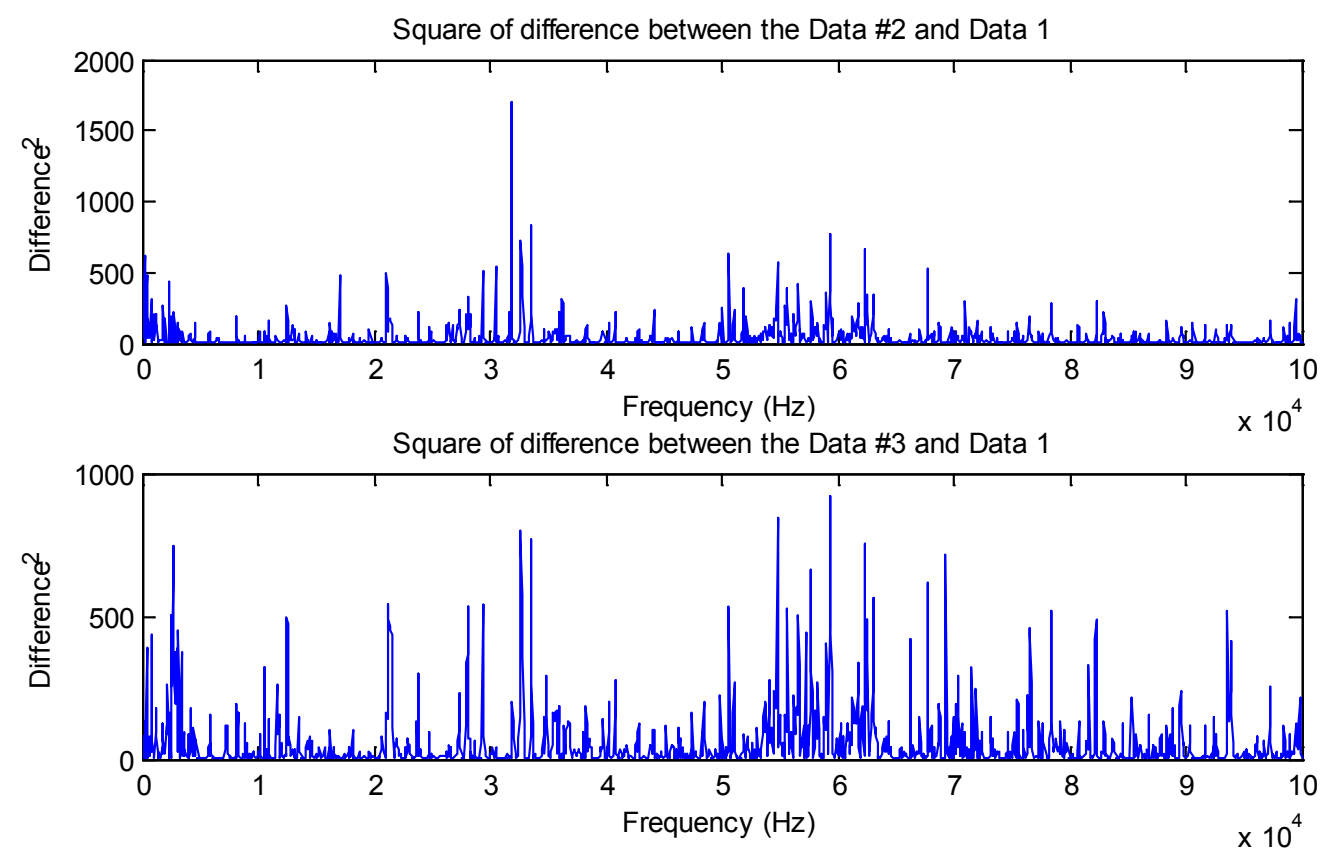

Figure 2.15 - Square of difference between perfect pipe and pipe with small and large hole.

To validate the upper results, bar graphs of sum of square of difference between the each test case (defect level) were plotted and can be seen in the figure2.16. The baseline of the plot (bar 1) is the data obtained from the perfect copper pipe (pipe 1). The other two bar graphs are the square of difference of the small hole and large hole data from the baseline (reference data from perfect pipe) respectively. A great amount of change can be seen from the figure. The first bar will always remain zero irrespective of the defect size and shape. The other two bar graphs depend on the intensity of the defect. As it can be seen that the difference between each bar graph is 
easily distinguishable, this difference is only because of the severity of the defect increases at every stage. Bar graph 3 represents the pipe with the bigger hole at the center, 2 represent the smaller hole at the center and 1 is for the perfect pipe.

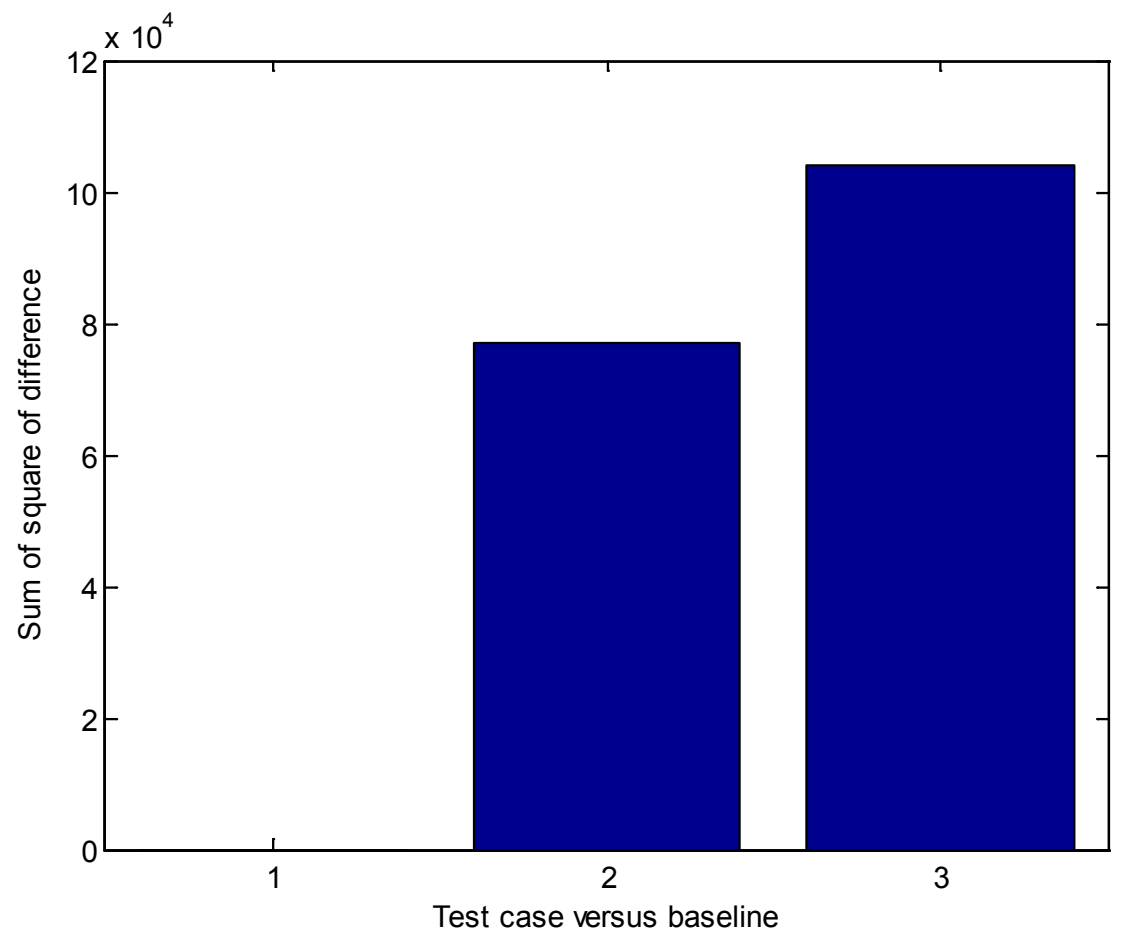

Figure 2.16 - Sum of square of difference between perfect pipe and each test case; perfect pipe/reference data (1), small hole (2) and largehole (3)

Figure 2.17and figure2.18clearly shows that the difference of the sum of the squares can be seen maximum in the fourth frequency interval (50 $\mathrm{KHz}-1000 \mathrm{KHz}$ ). The plot is divided into four frequency level intervals starting from $5 \mathrm{KHz}$ to $25 \mathrm{KHz}$ with $25 \mathrm{KHz}$ of increment at every step. Plot in the fourth interval has the maximum values for the difference among the data. It is therefore concluded with the help of plots that a small change in 
the geometry of the specimen (pipe) can be easily detected and also we can distinguish between the small defect and large defect.
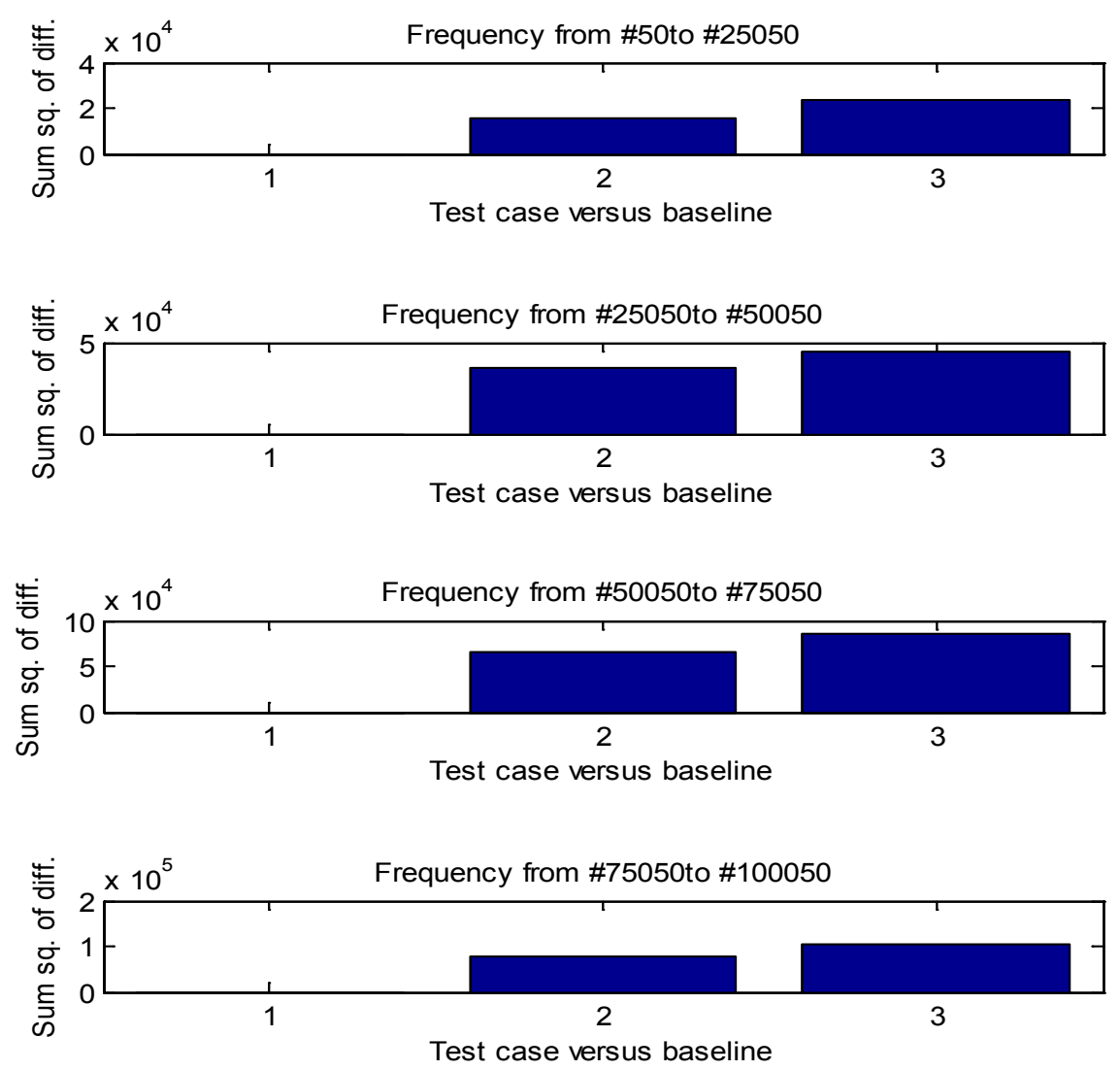

Figure 2.17 - Sum of square of difference of each test case at four different frequency levels 


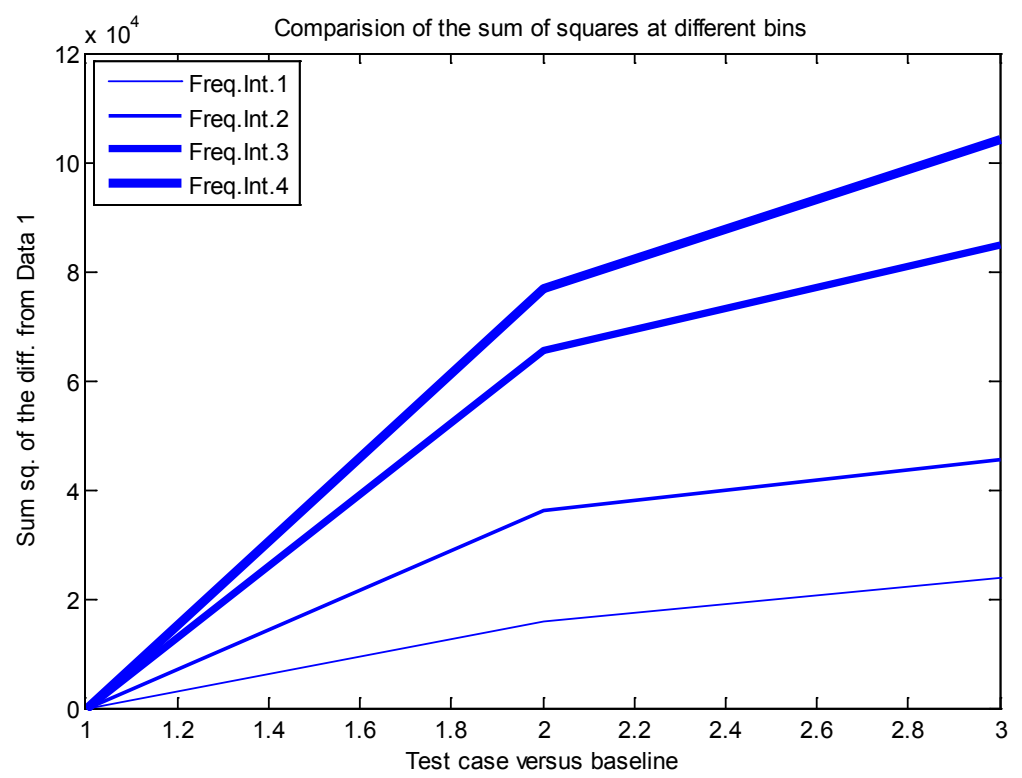

Figure 2.18 - Test case v/s sum of square of difference between each test case and baseline at four frequency intervals.

\subsubsection{Copper pipe with dent at the center}

The third set of experiment was performed on pipe 3. A dent was created at first stage by pressing the pipe with the help of hand held pliers. Dent is made at the center of the pipe and it just simulates the concentrated load on the pipe. This is done to show that how the pipe will going to behave if it comes under some concentrated load. An unprocessed signal was extracted from the pipe with the help of signal analyzer and can be seen in the figure 2.19. The signal came out to be very unclear and no such difference can be made until the data from this received signal will be processed by using various statistical algorithms capable of extracting useful damage indices from the measured data [22, 23]. 


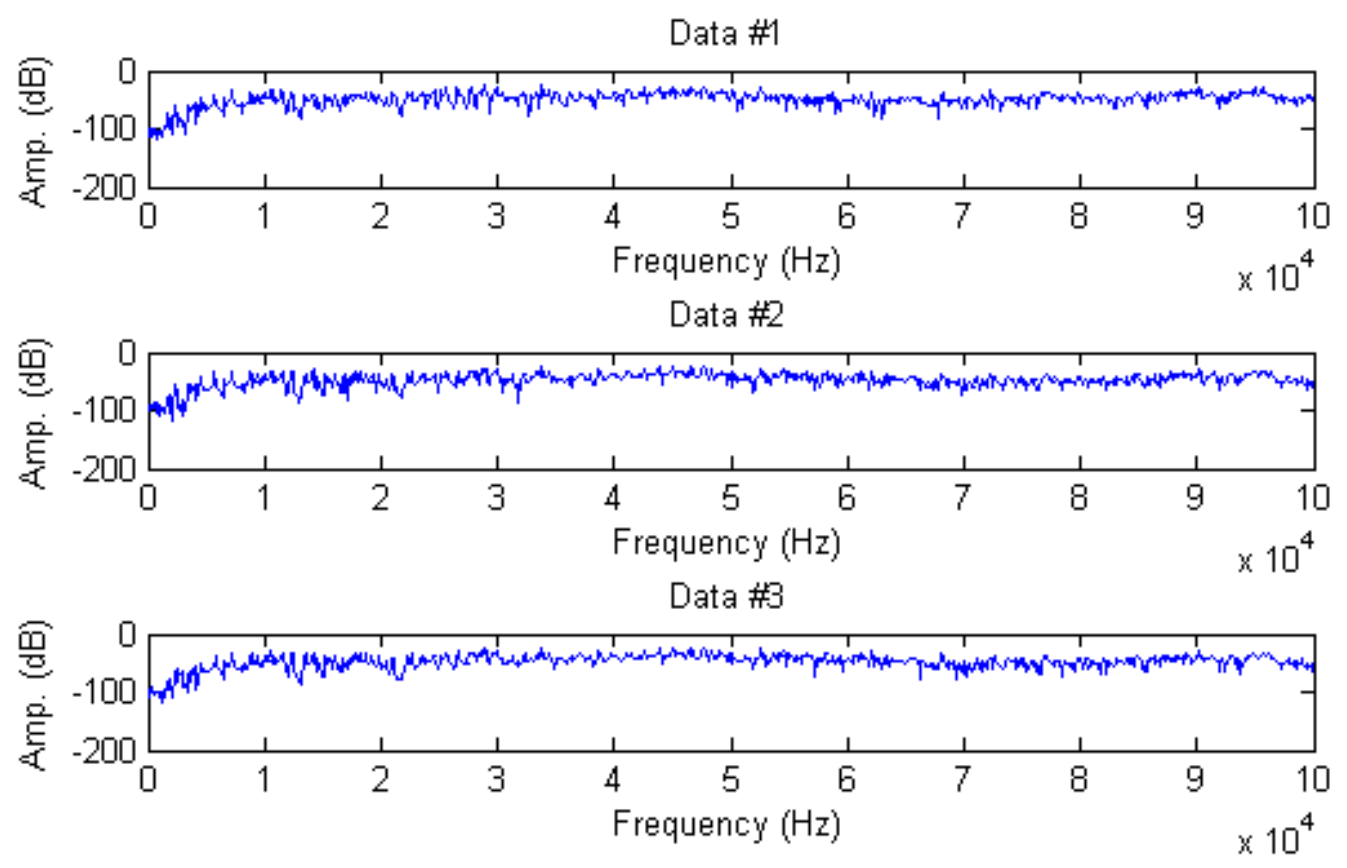

Figure 2.19 - Spectrum analysis perfect pipe, pipe with small and large dent

Further the comparing of two states of the structure was executed in a linear fashion. The data which we obtained from the signal analyzer provides the useful information regarding the health of the host structure. However, this data in its original state does not provide quantifiable information regarding the presence and state of damage within the structure. Therefore, processing the measured signal, a feature needs to be extracted, which is sensitive to the damage and insensitive to other parameters. Square of difference between the two data sets was calculated and plotted in figure2.20. The top window portrays the difference between the perfect pipe (data 1) and first level dent (data 2), the second window shows the difference among data 1 and second level dent (data3). Though the difference among the two 
spectrums is not very convincing except at around $60 \mathrm{KHz}$ where the difference among the two is not very large but still it is prominent. The difference among the signals grows larger with increase in the magnitude of defect.
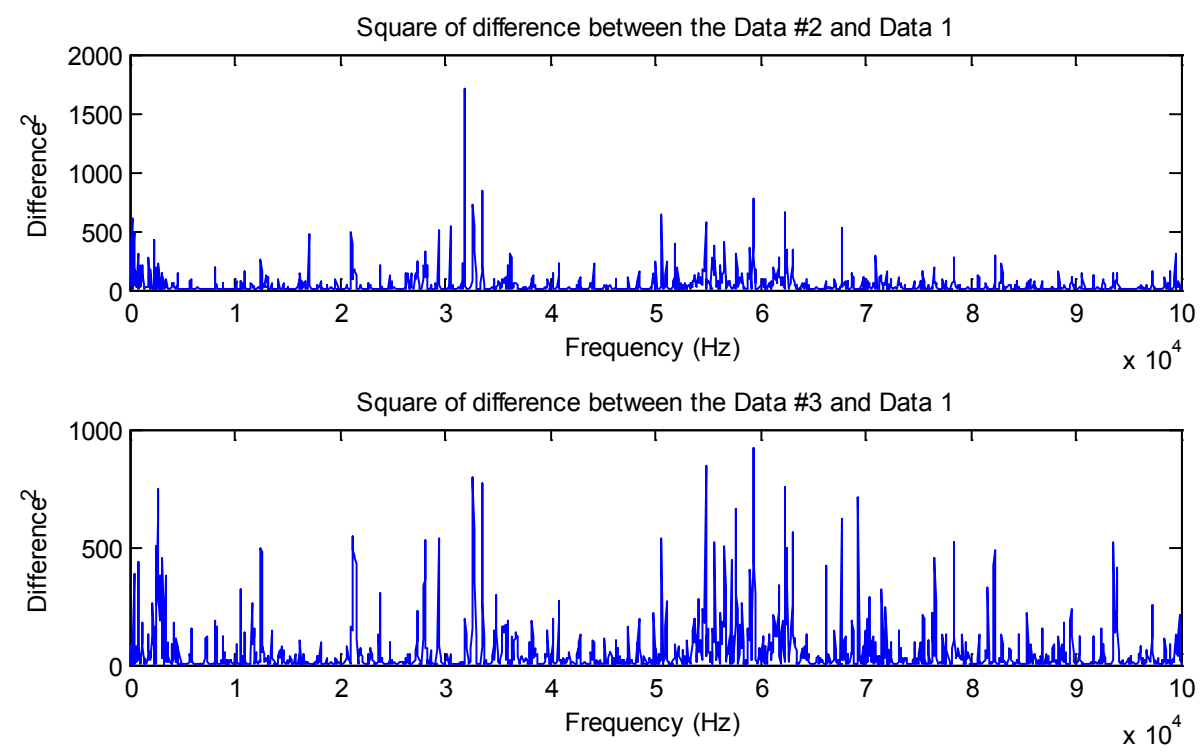

Figure 2.20 - Square of difference between perfect pipe and pipe with small and large dent

To have a closer look at the severity of defect in the pipe, the sum of square of difference for the two cases of defects was plotted against the reference (perfect pipe). The bar 1 is for the perfect pipe that is why its value is nil and the other two are for the defect size 1 and defect size 2 . The same set of result can be shown through the time line graph, which is presented in figure 2.21. Here the four set of lines represents the magnitude of the defect at four different frequency distributions. The best result is shown in the 
fourth frequency interval as the value of sum of square of difference matches with that in figure 2.22 , which is $10 \times 10^{-4}$ for the final defect.

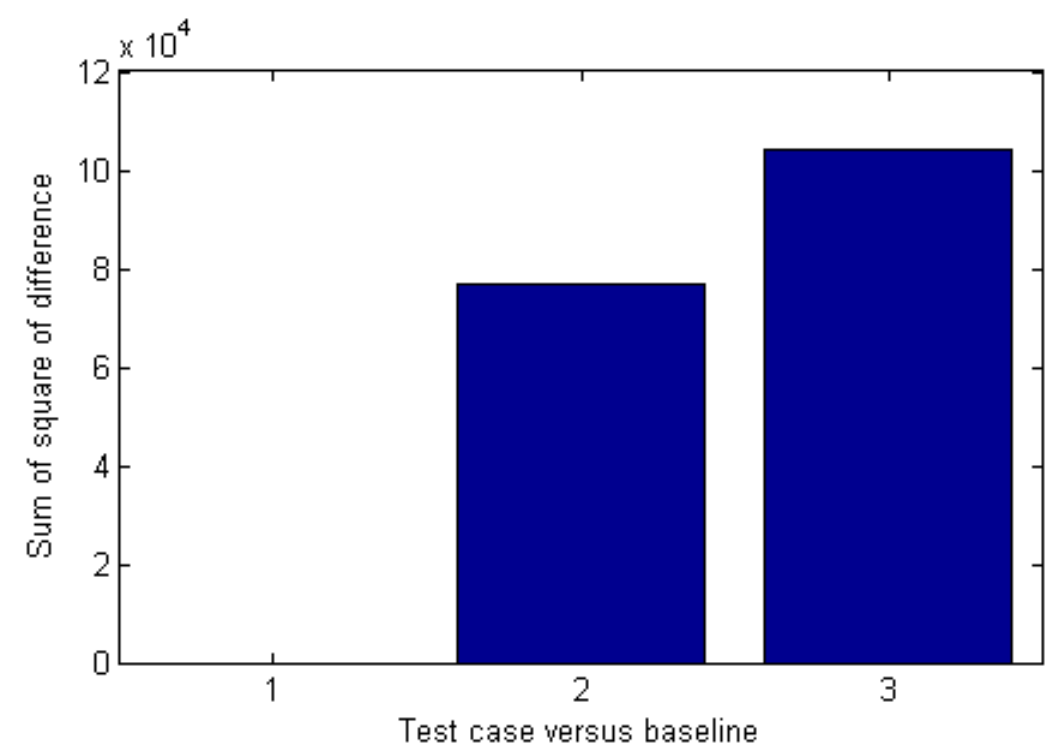

Figure 2.21 - Sum of square of difference of each test case; perfect pipe/reference data (1), small dent (2) and large dent (3)

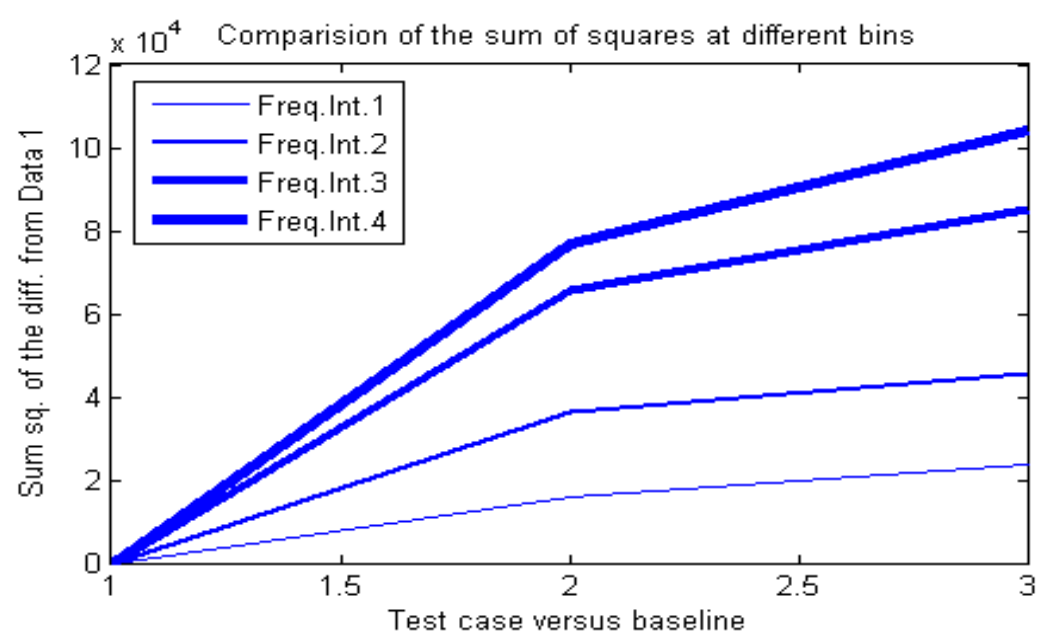

Figure 2.22 - Test case v/s sum of square of difference between each test case and baseline at four frequency intervals. 


\subsubsection{Lamb wave approach}

After testing, receipting and interpreting the data by sweep sine wave approach, lamb wave method (approach) was applied to the same copper pipes which were used for sweep sine wave method. Metis Design SHM Node MD 75602 equipment was used to create the lamb waves at one end of the pipe and receiving the signal from the other end. Signal was recorded after each level. Three defect levels were tested and recorded successfully.

\subsubsection{Copper pipe with cut at the center}

Pipe with cut was first tested followed by the pipe with hole and dent. When the piezoelectric element (marked 2) is excited from one end it generates a voltage which correlates the power with which the lamb waves were received at the other end by another piezoelectric element (marked 2). The piezoelectric element was excited at $200 \mathrm{KHz}$ as well as at $300 \mathrm{KHz}$ in order to see that at what frequency level the signal coming out to be clear and meaningful. The envelopes of the signal at $200 \mathrm{KHz}$ and $300 \mathrm{KHz}$ were calculated and plotted as shown in figure 2.23 and 2.24 respectively. The plot at $300 \mathrm{KHz}$ excitation frequency seems to be more clear and detailed than that with $200 \mathrm{KHz}$ excitation frequency. The signal with $300 \mathrm{KHz}$ excitation frequency is more symmetric and systematic for all the three cases (perfect, cut1 and cut 2); the only change seen is that the amplitude values at some points are extremely different. This can be possible due to the defects present in the pipe. This is again will be explained with more details as we move 
down on the lamb wave approach. So, for further processing the data, 300 $\mathrm{KHz}$ frequency was chosen to excite and receive the signals from the copper pipes.

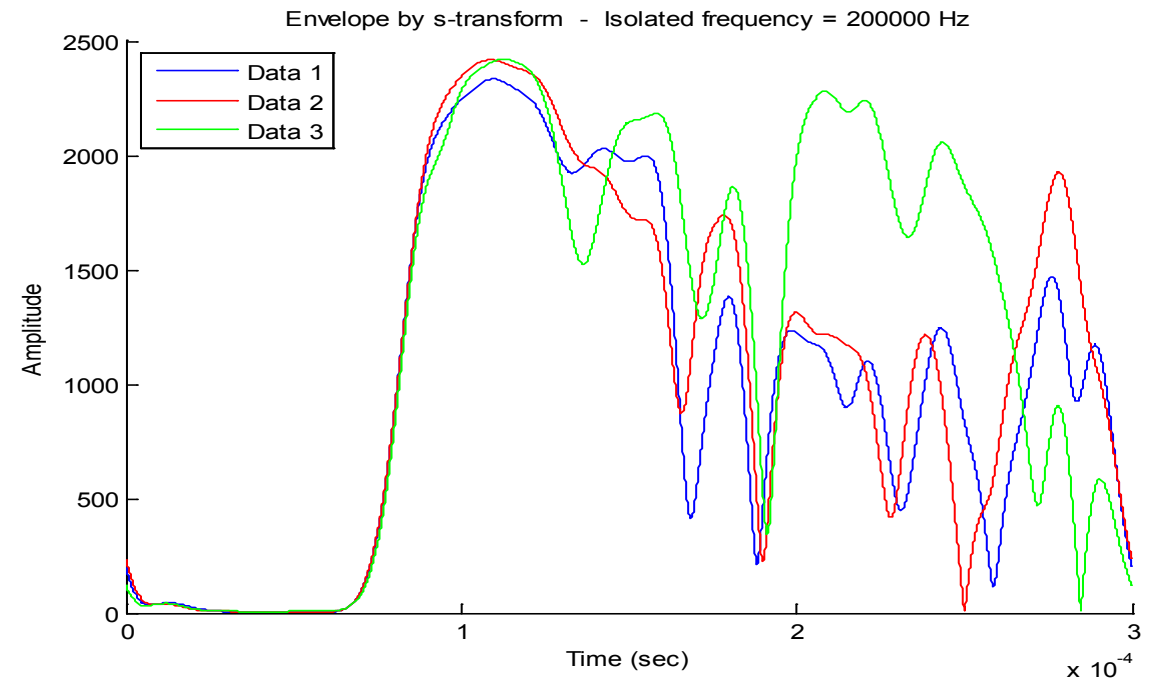

Figure 2.23 - Variation of the envelope signal with simulated pipe with defect when the $200 \mathrm{KHz} 3.5$ sine wave is applied. The perfect pipe (Data 1), pipe with first cut (Data 2) and second cut (Data 3)

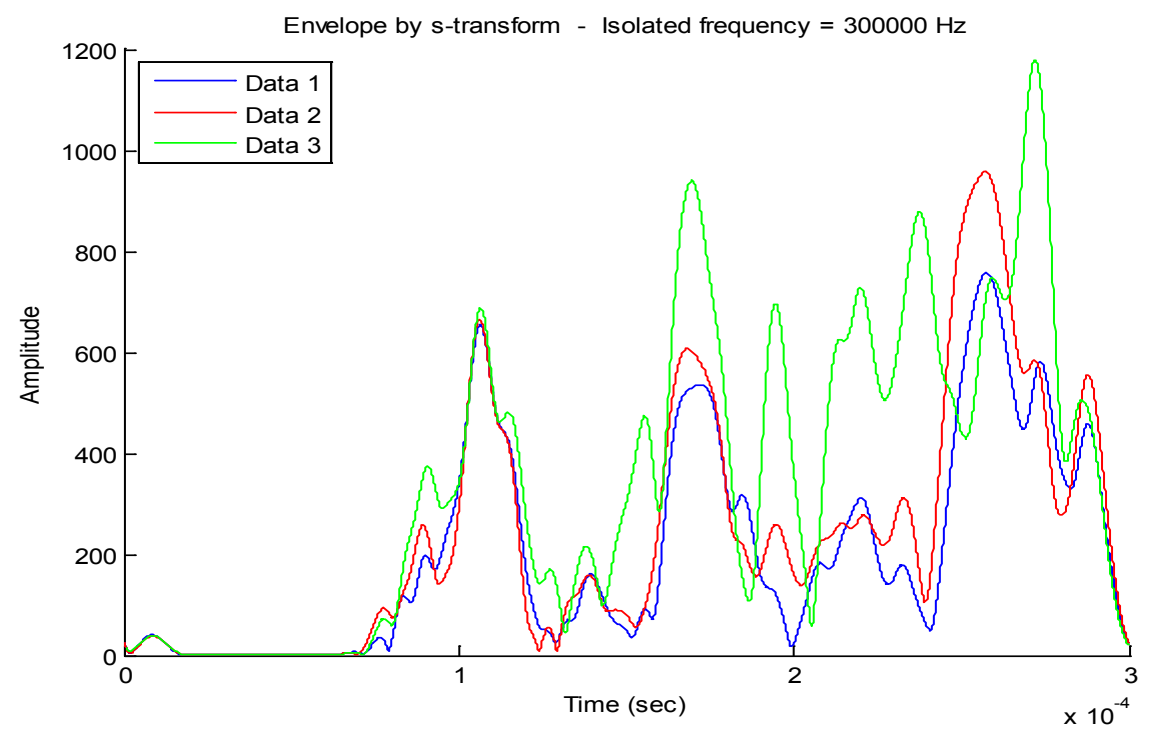

Figure 2.24 - Variation of the envelope signal with simulated pipe with defect when the $300 \mathrm{KHz} 3.5$ sine wave is applied. The perfect pipe (Data 1), pipe with first cut (Data 2) and second cut (Data 3) 
The piezoelectric element was excited at $300 \mathrm{KHz}$ and the time domain signal was received at the other end and is shown in figure 2.25. The signal from all three levels can be seen in the figure. The signal looks very similar but still the difference can be seen when observed carefully.
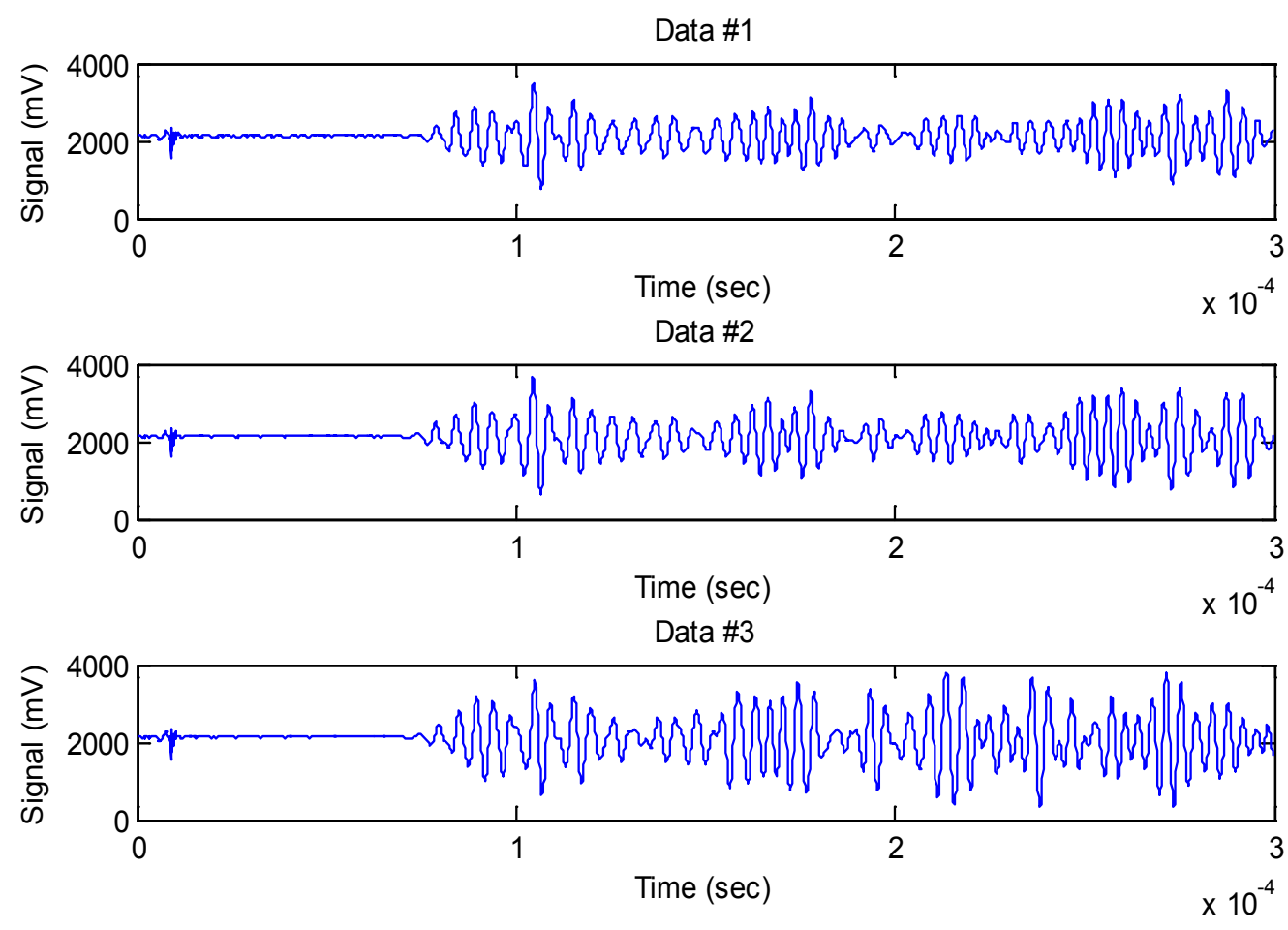

Figure 2.25 - The received signal at sensing piezoelectric element.

The data obtained is in unprocessed form and needs to be further processed to obtain some useful information from it. So, first of all $\mathrm{S}-$ transformation of the signal was done to check for the critical areas. S transformation was an extension of the continuous wavelet transformation (CWT). It was introduced by Stockwell as a "phase correction" of CWT. It can 
provide frequency dependent resolution, while maintaining a direct relationship with the Fourier Spectrum [40].S - transformation can detect the changes in the characteristics of a system in the presence of noise [41]. S - transformation can also be used to detect the propagation features of the lamb waves on a plate structure, and identify the occurrence of damages by the delay time and echoes [42]. S - transformation has the desirable characteristics that continuous wavelet transformation lacks, and it can give a more sound time-frequency representation of sensory signals [43]. The $\mathrm{S}-$ transformation plots of the received signal for all three cases (perfect pipe, pipe with cut 1 and pipe with cut 2) are shown in figure 2.26. It can be seen from the plots that the red circle which is at $2.5 \times 10^{-4}$ seconds and between $250 \mathrm{KHz}-300 \mathrm{KHz}$ range moved towards right from its initial position. The only area of interest is the area with red patches because the patches with yellow and green patches are due to the presence of some electronic device near the system. So, our main goal is to observe the red patch. For the third case (cut 2) at around $2 \times 10^{-4} \mathrm{sec}$. the red patch suddenly appeared which is not visible for the other two cases. This can be an indication that the defect size is increasing and can be taken into consideration. The $\mathrm{S}-$ transformation is very basic technique to check at what time intervals the signals are separating. 


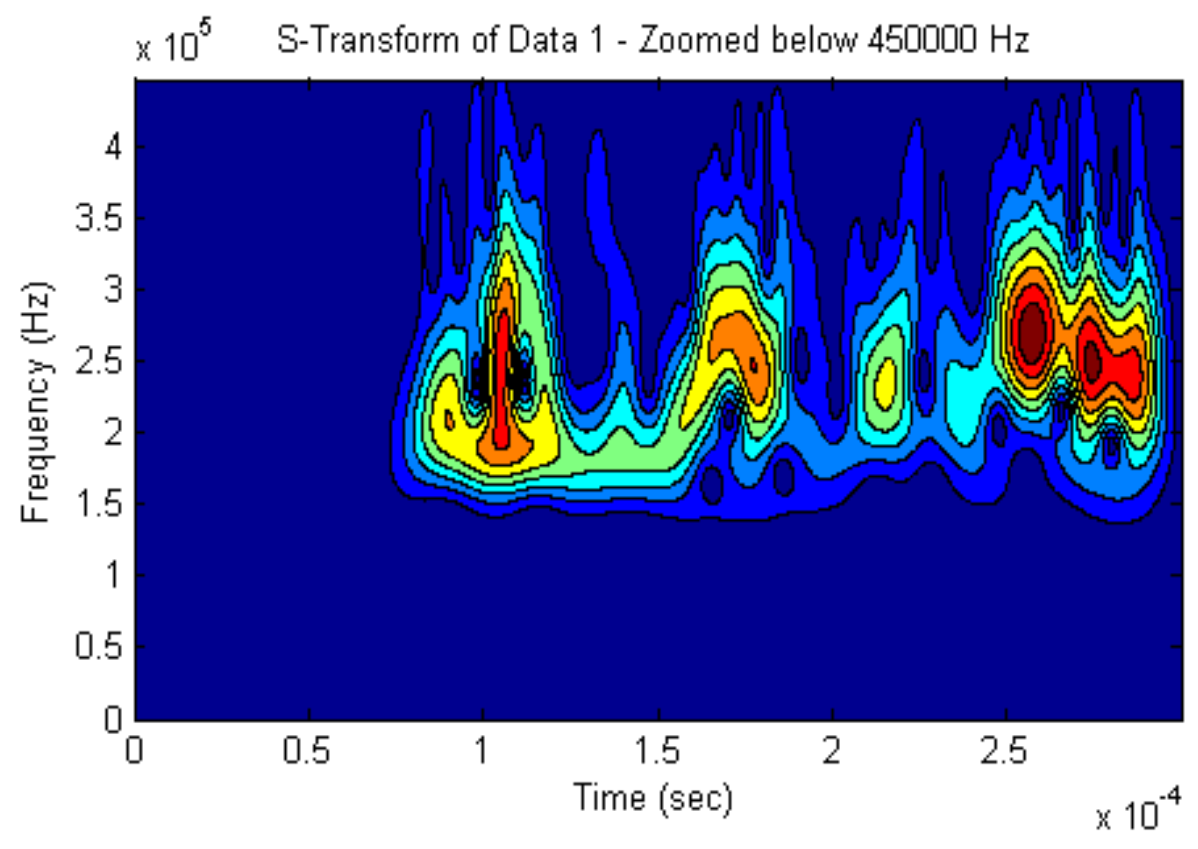

(a)

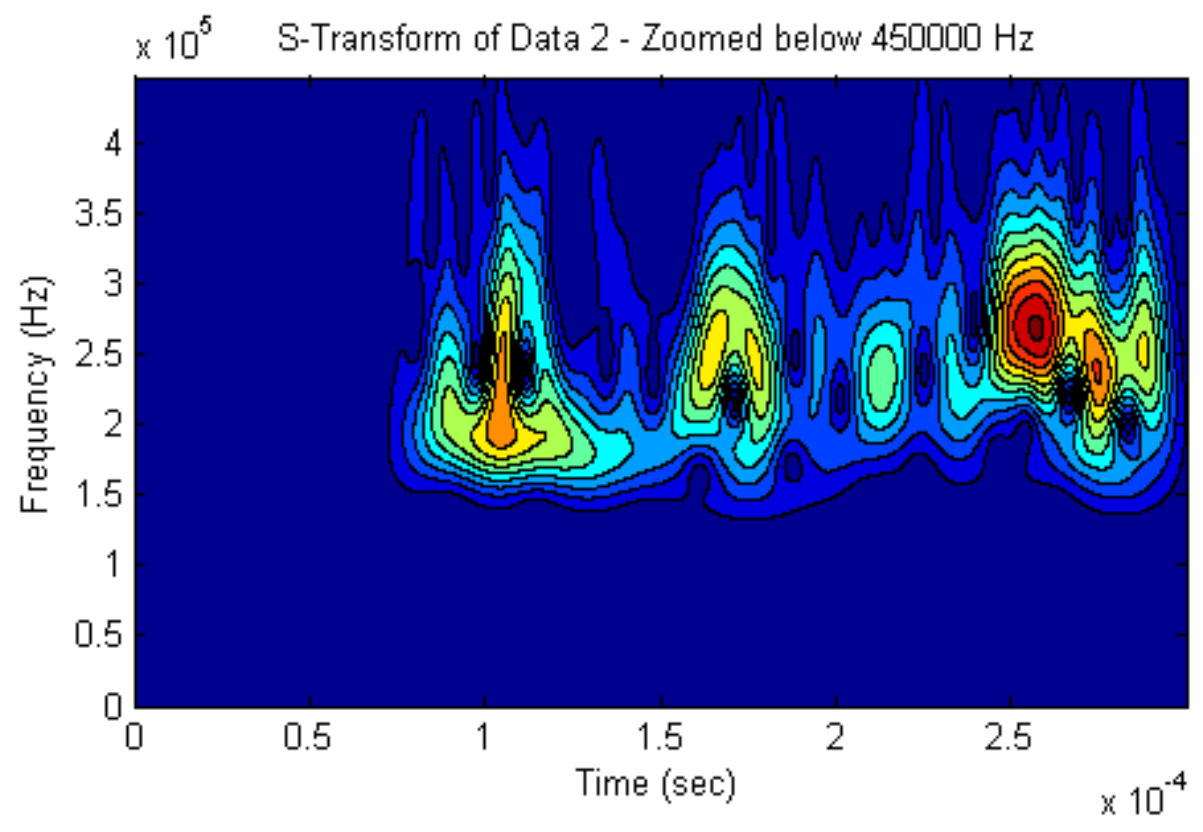

(b) 


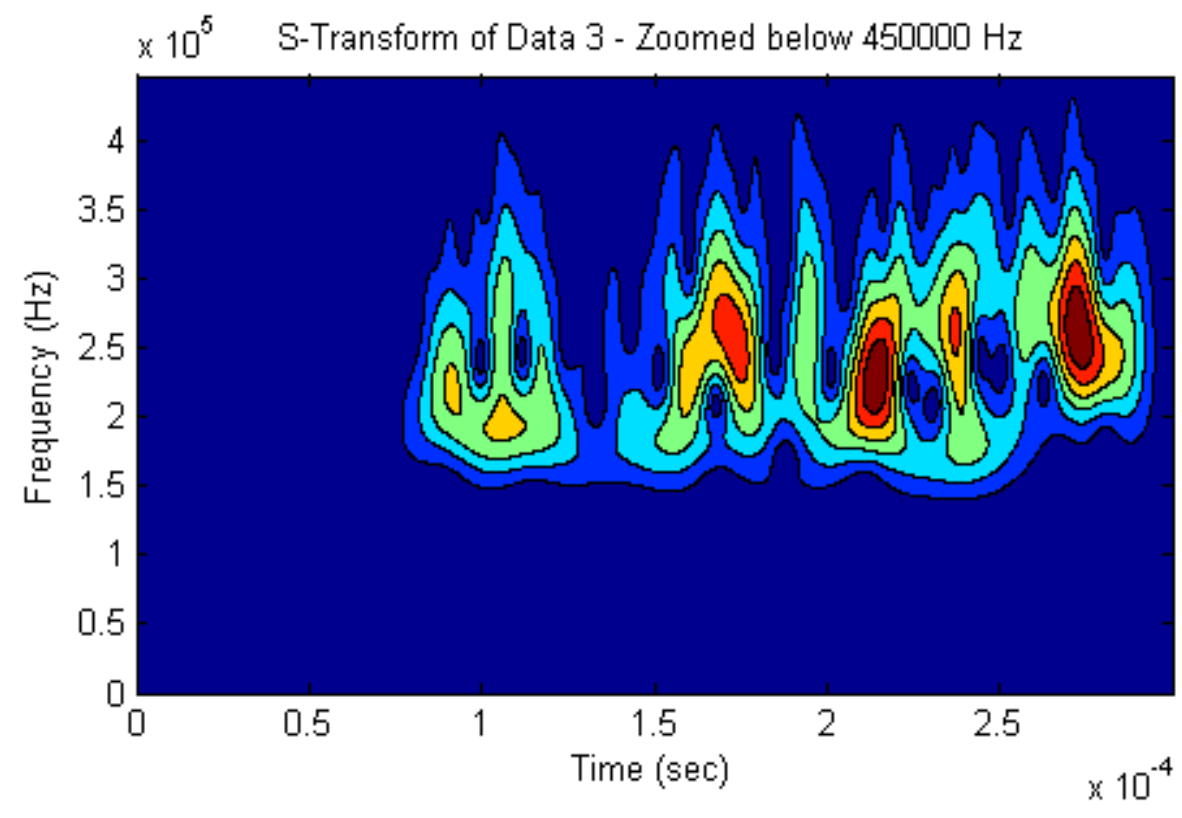

(c)

Figure 2.26 - S-transformation plots below $450 \mathrm{KHz}$ frequency (a) perfect pipe, (b) pipe with first center cut and (c) pipe with through center cut

To have a close look at the signals, the envelope of the $\mathrm{S}-$ transformation were calculated and plotted as shown in figure 2.27. It can be seen from the figure that at about $150-200 \mu$ sec there is a significant difference between the peaks of all the three cases. As it can be seen from the figure that all three signals were moving almost on the same path up to 150 $\mu$ sec, after this time period the signals started splitting from each other. The reason could be the defect present at the center of the pipe. Now, for the first case (perfect pipe, data 1) the amplitude of the signal at around $150 \mu \mathrm{sec}$ is below 600 whereas for cut 1 the amplitude at same time increases to 600 and for cut 2 it even increases significantly to around 900 . The reason can be the presence of defect. It can be seen clearly that with the increase in the defect 
size the amplitude value at $150-160 \mu$ sec increases as well. Now the reason for which we choose $150-200 \mu$ sec range can be related to the length of the pipe we used to perform the experiments. The length of the pipe was 12 inch but the distance between the piezoelectric elements was 10 inch and the total time taken by the signal to propagate from one end of the pipe to other was $300 \mu \mathrm{sec}$. The location of the defect was at the center of pipe (at around 5 inch from each sensor). That means the lamb wave could reach to the defect location in about $150 \mu \mathrm{sec}$. This was the only reason for selecting the range of time from $150 \mu \mathrm{sec}$ to $200 \mu \mathrm{sec}$. The results obtained betweenthis time frame are promising as the amplitude consistently grows up which directly corresponds with the increase in defect.

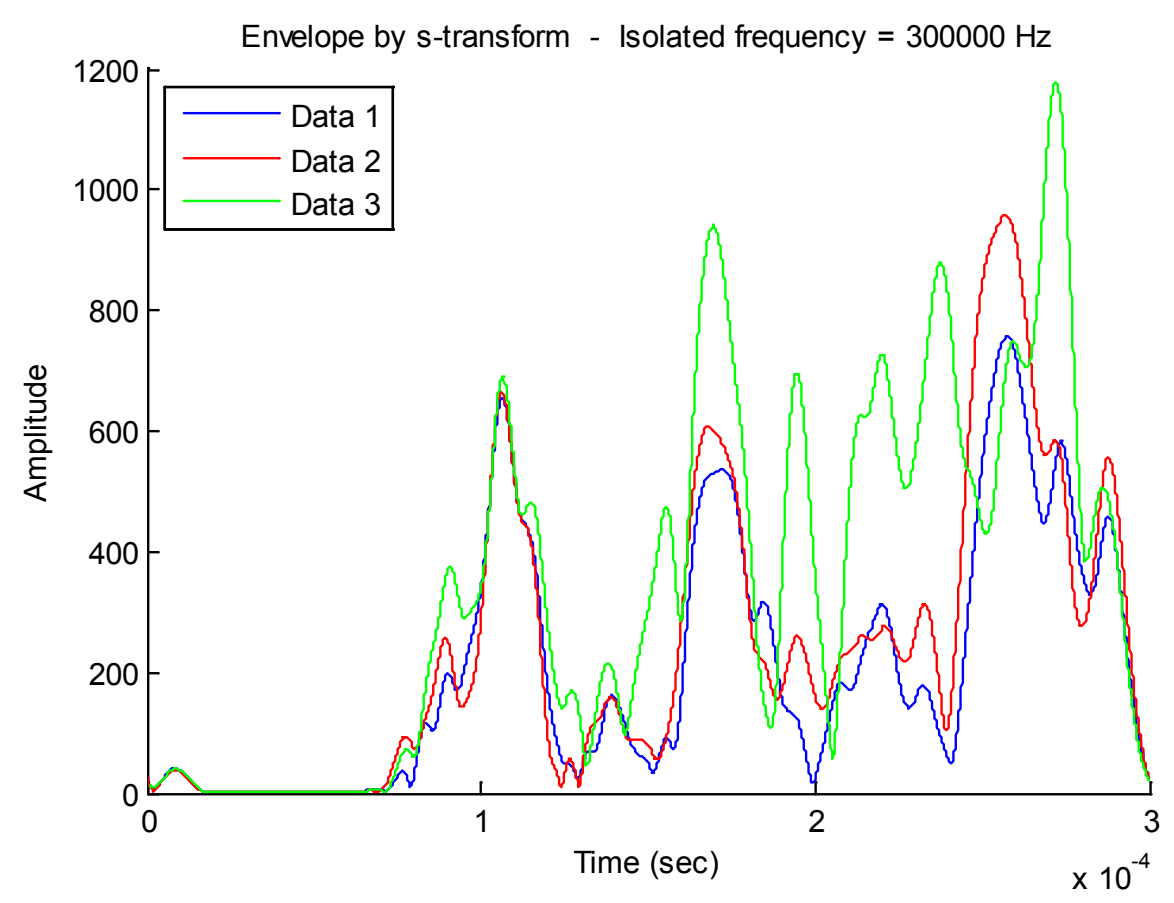

Figure 2.27 - Envelope of the S-transformation of the signal. Perfect pipe (data 1), pipe with first center cut (data 2) and through center cut (data 3) 
After taking the envelope from the $\mathrm{S}$ - transformation, envelope of each signal will be calculated. The envelope of data 1 (perfect pipe) is plotted and shown in figure 2.28. The main objective of doing this is to check that weather we can decrease the data points without increasing the error. The data points of the envelope were decreased by 4 set of levels and average error was calculated at each level.

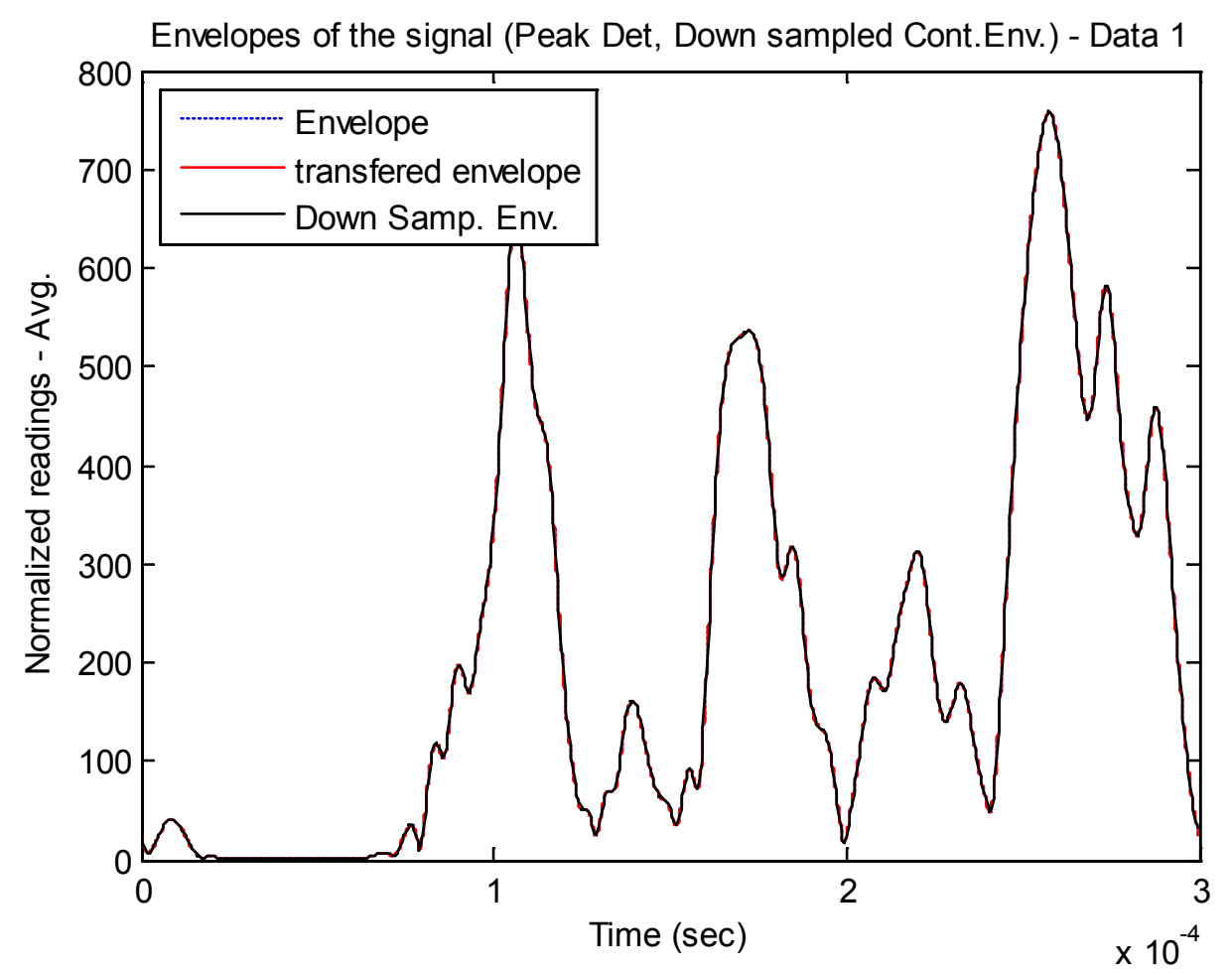

Figure 2.28 - Envelope of the original signal from perfect pipe

The envelope of the original signal was calculated by wavelet transformation and its first level approximation was done followed by the average error between the envelope of original signal and the approximate signal as shown in figure 2.29. It can be seen from the figure that the 
envelope of the original signal is less than 600 data points and the approximate signal at first level came under 300 data points with very minute average error of 0.1 percent.
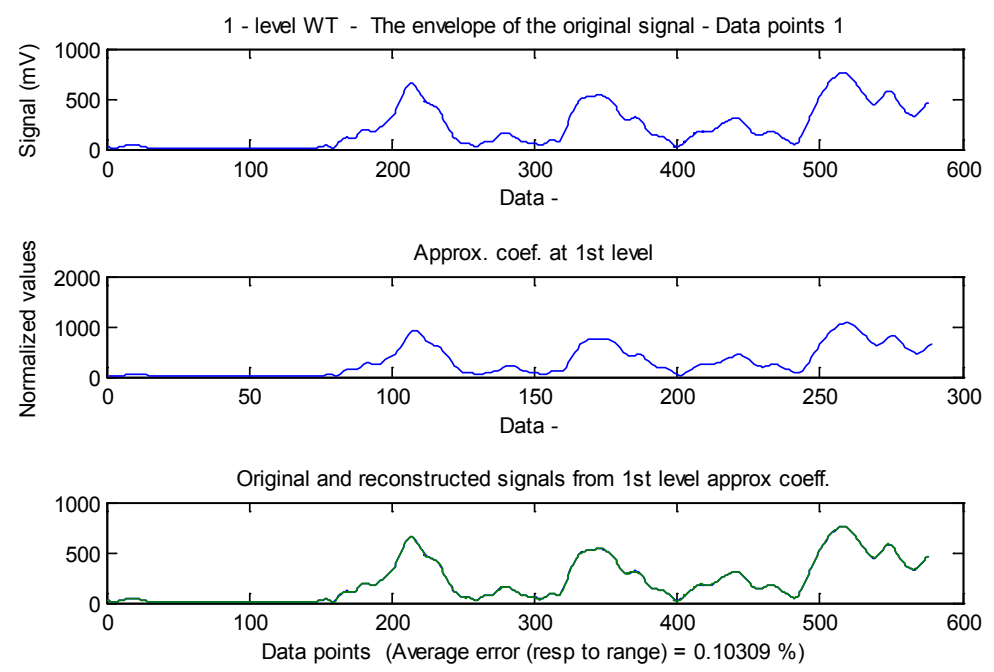

Figure 2.29 - Wavelet transformation of the original signal, its first level approximation and average error.

The second level approximation of the envelope of original signal is shown in figure 2.30. Here the signal is reduced from 600 points to 150 points with 0.35 percent average error. The error is still below one percent which means that we can consider the approximate signal for further calculations. 

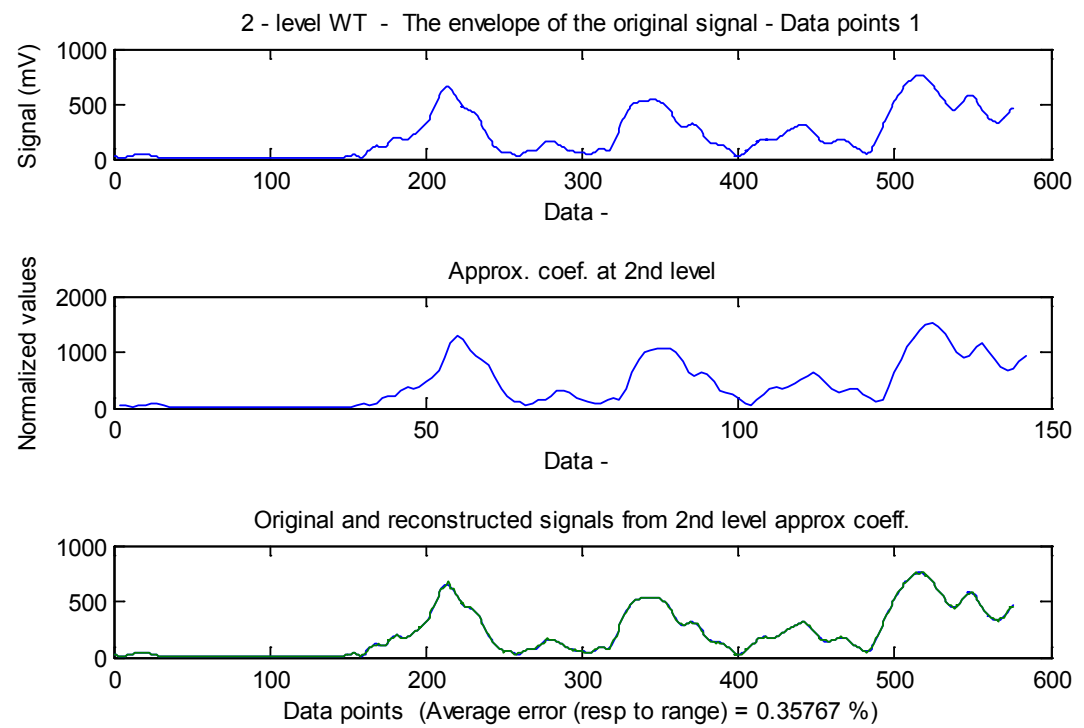

Figure 2.30 - Wavelet transformation of the original signal, its second level approximation and average error.

The third and fourth level approximation of the original signal is shown in the figure 2.31andfigure 2.32. In third level approximation the signal points were reduced from 600 to 80 but the average error came out to be 1.18 percent and being above one percent this signal is not considered to be feasible for further calculations. The average error of the fourth level approximation is significantly higher than one percent, which is 3.3 percent and is definitely can never be considered for further processing the calculations though the data points are considerably decreased from 600 to 40. 

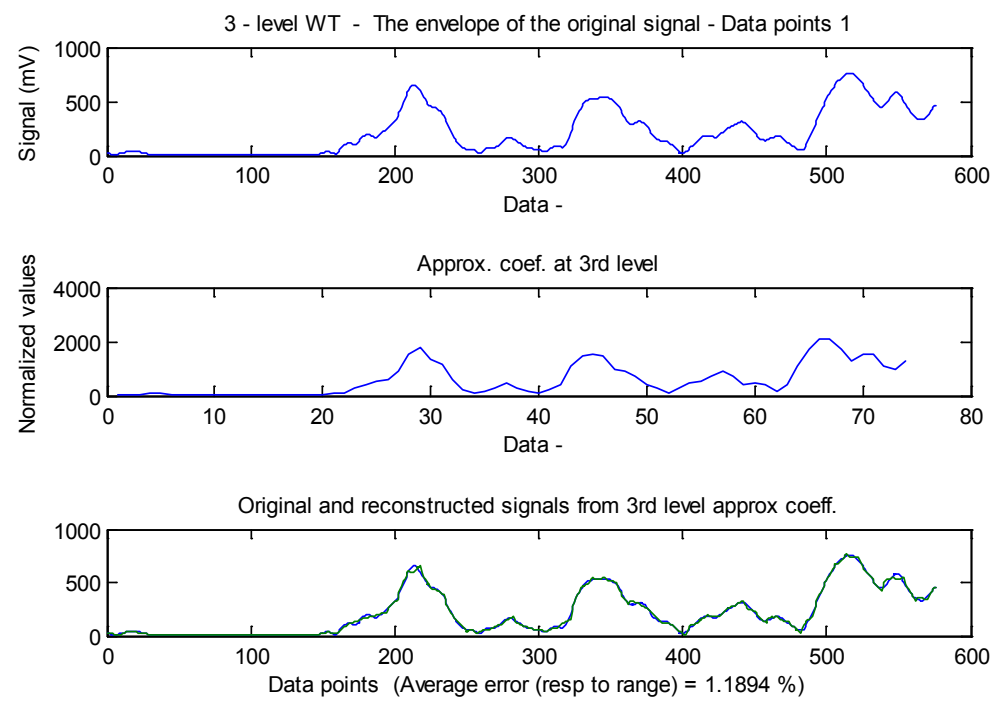

Figure 2.31 - Wavelet transformation of the original signal, its third level approximation and average error.
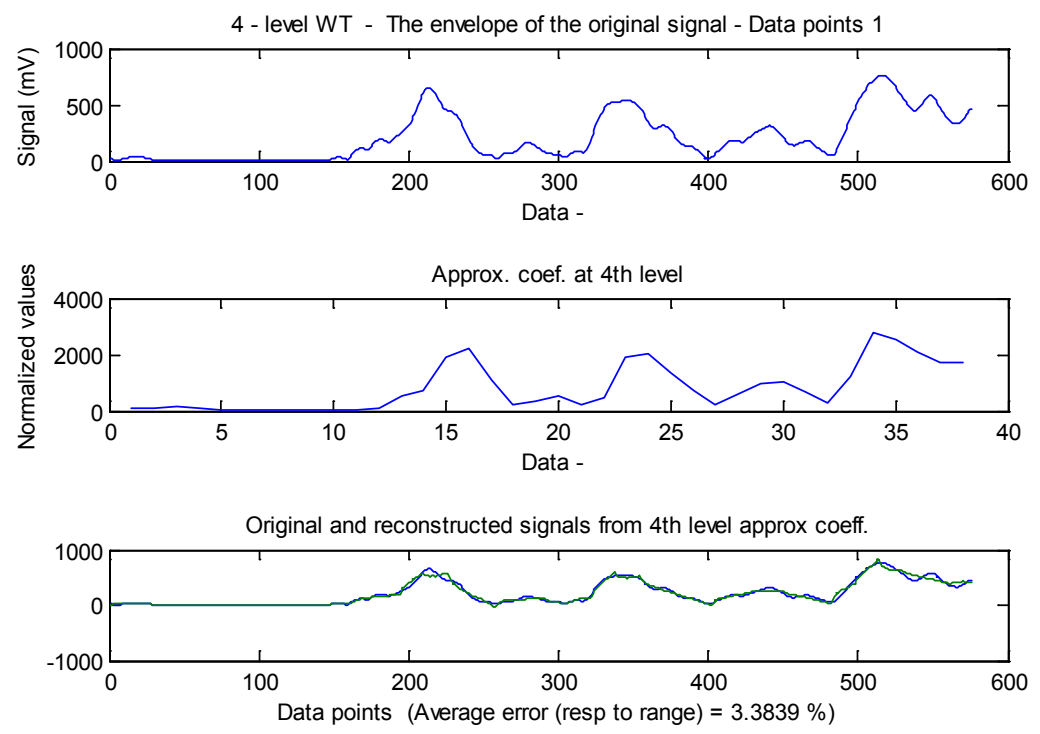

Figure 2.32 - Wavelet transformation of the original signal, its fourth level approximation and average error.

The advantage of approximating the envelope of the original signal is to reduce the data points of the signal in order to reduce the processing time. In our experiments the pipe sample used was 12 inch in length but in real 
applications the pipe can be from few hundred feet to hundred meters and for that more data points will be needed to capture the signal with maximum details. Process such huge data sets needs advanced equipment which consumes less time to process the data. But by approximating the original signal under allowed average error, we can save a lot of time and money by using less advanced equipment thus, making the whole process to be very economical without compromising the accuracy of the received signal.

The envelope of the data 2 (cut 1) and data 3 (cut 2) was calculated and can be seen in figure 2.33 andfigure 2.34 .

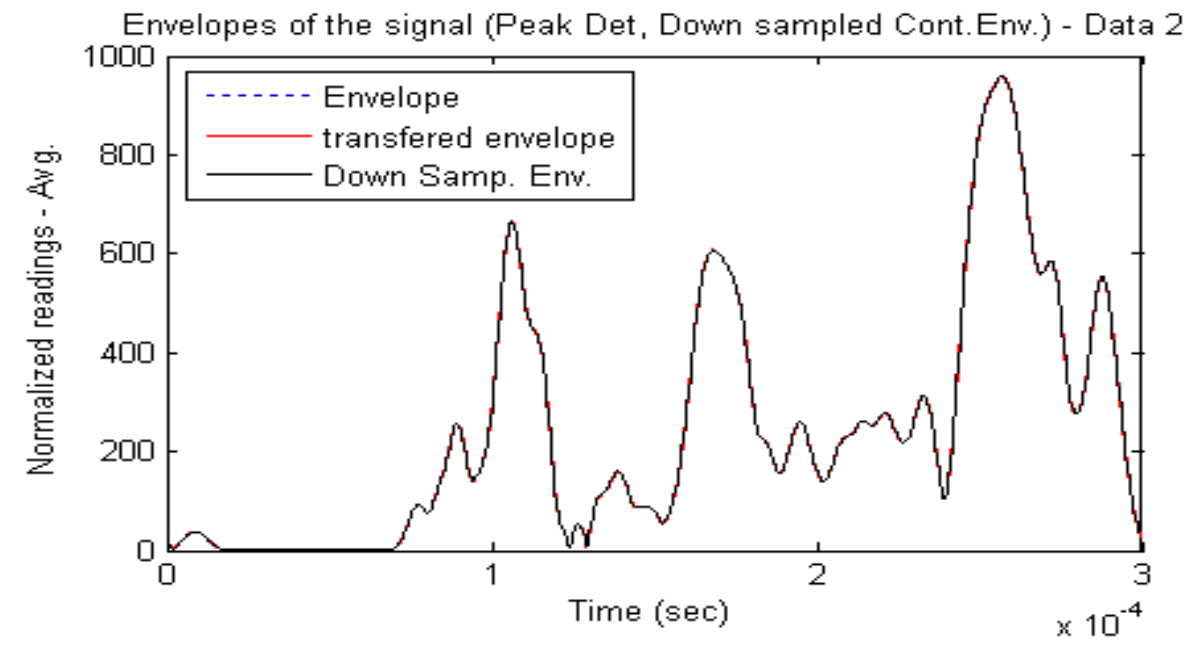

Figure 2.33 - Envelope of the original signal from the first center cut. 


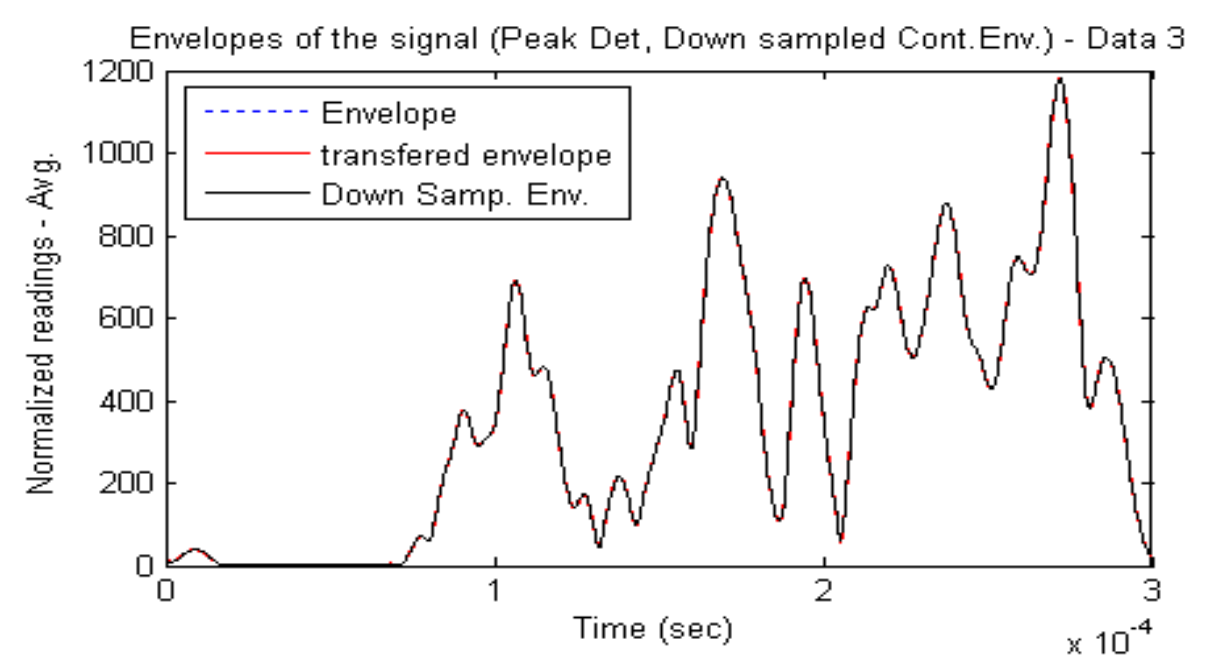

Figure 2.34- Envelope of the original signal from the second cut.

Similarly, the approximate coefficient of the envelope of the original signal was calculated and the acceptable value of absolute error for both cases (pipe with cut 1 and cut 2) came out to be 0.29 percent and 0.38 percent at second level of approximation respectively. The plots for the approximation of these two cases can be seen in figure 2.35 andfigure 2.36 .

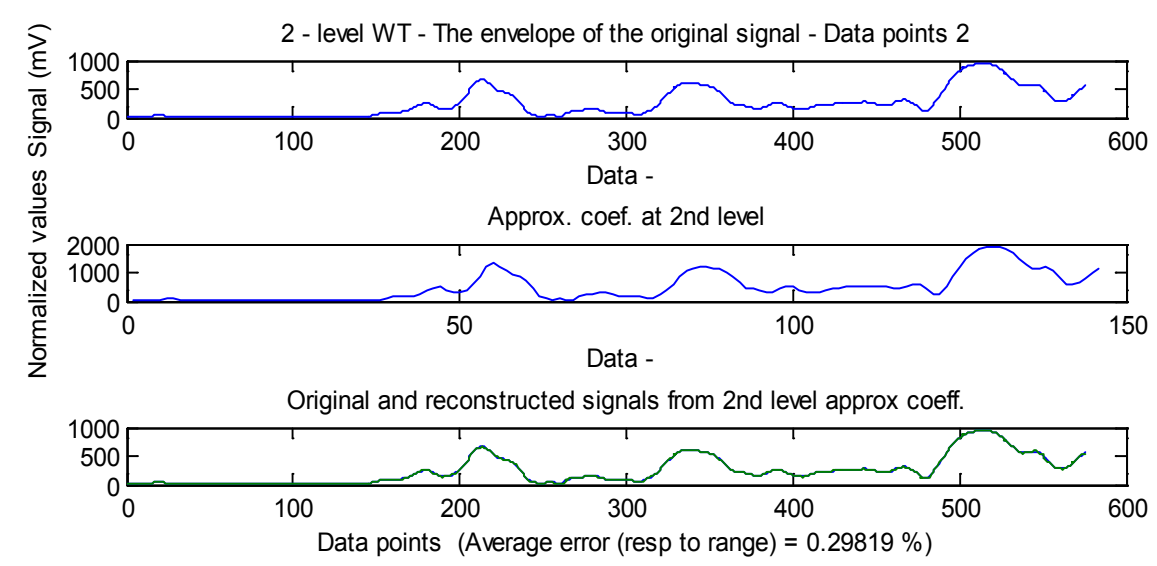

Figure 2.35 - Wavelet transformation of the original signal from first cut, its second level approximation and average error. 


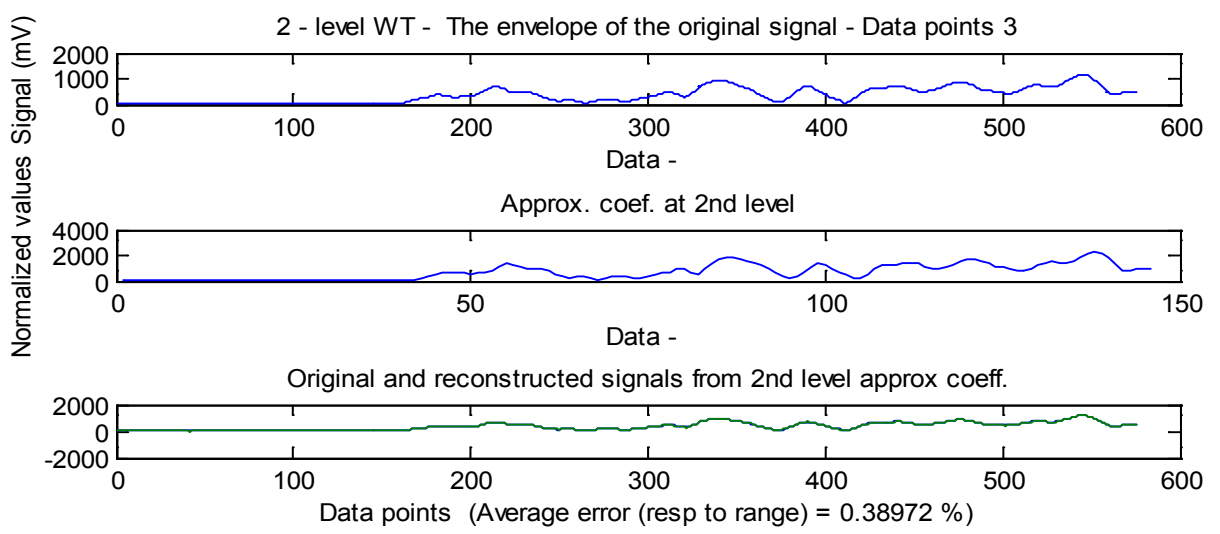

Figure 2.36 - Wavelet transformation of the original signal from second cut, its second level approximation and average error.

After fitting the envelope of the original signal to lower number of data points, comparison of the envelope of the signal was calculated from peak to peak in time domain. The absolute value of the difference between two signals was calculated and can be seen in figure 2.37, figure2.38andfigure 2.39. The basic principle behind comparing the signals was to see the difference between the each signal at each defect level. Figure 2.37 represents the difference of the reference signal (signal from perfect pipe) and signal at first defect level. Similarly, figure 2.38 shows the difference between the reference signal and the signal from second defect level. It can be seen from both the plots that the absolute difference for the second level defect is far more than that of first level defect. This clearly shows that the absolute difference in the signal increases as the defect level goes more severe. 


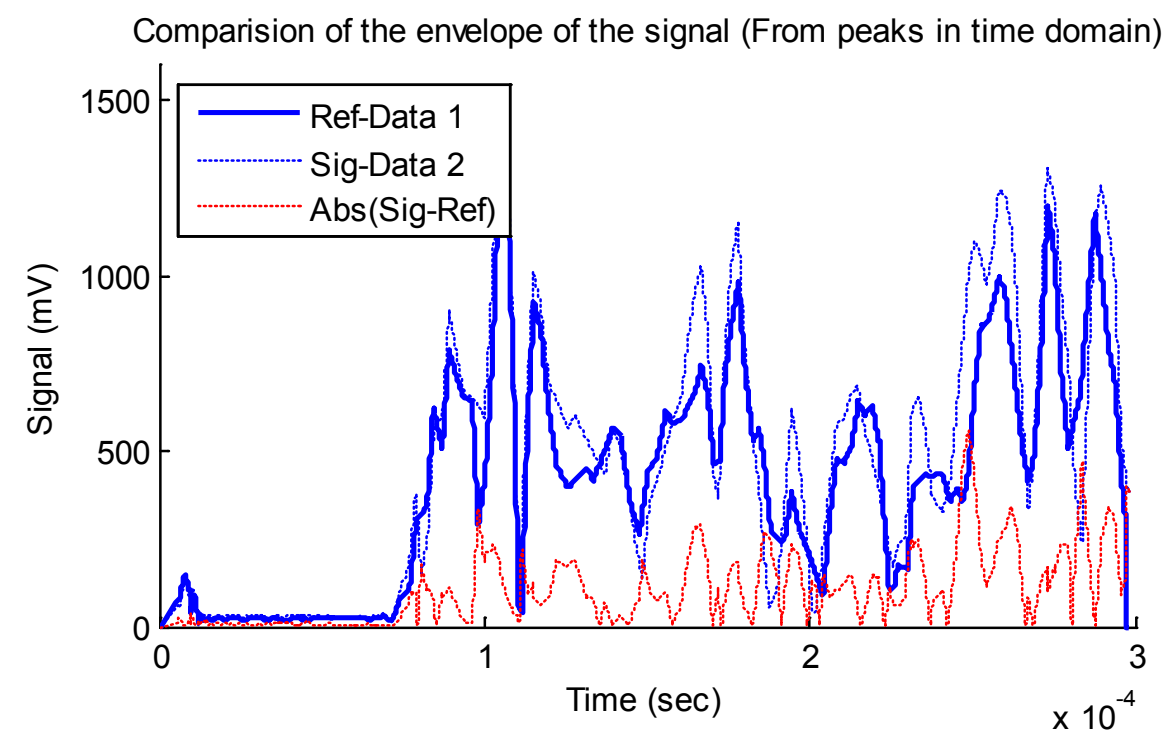

Figure 2.37 - Absolute difference of perfect pipe (data 1) and pipe with first cut (data 2)

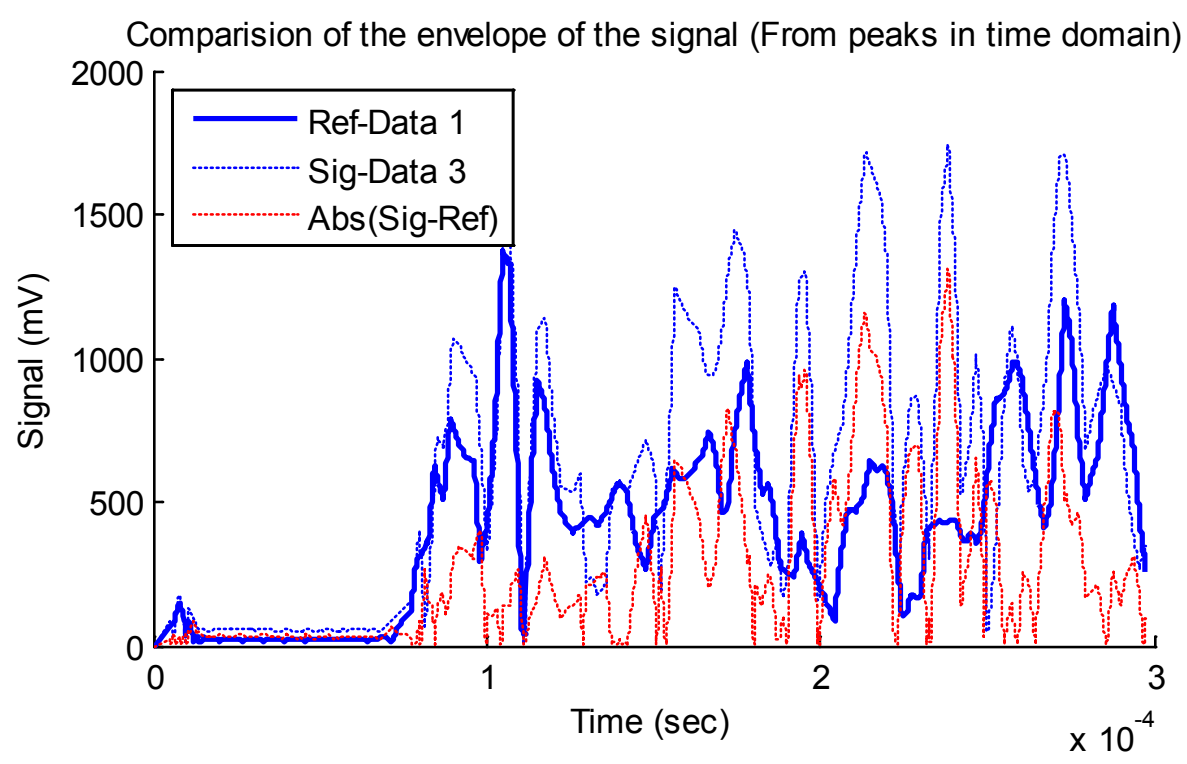

Figure 2.38 - Absolute difference of perfect pipe (data 1) and pipe with second cut (data 3)

The comparison difference between the two defect cases was calculated and shown in figure 2.39. In this case the reference signal was the signal 
from data 2 . Here it can be seen that the absolute difference in this case is little less than the first case (difference between data 1 and data 2).

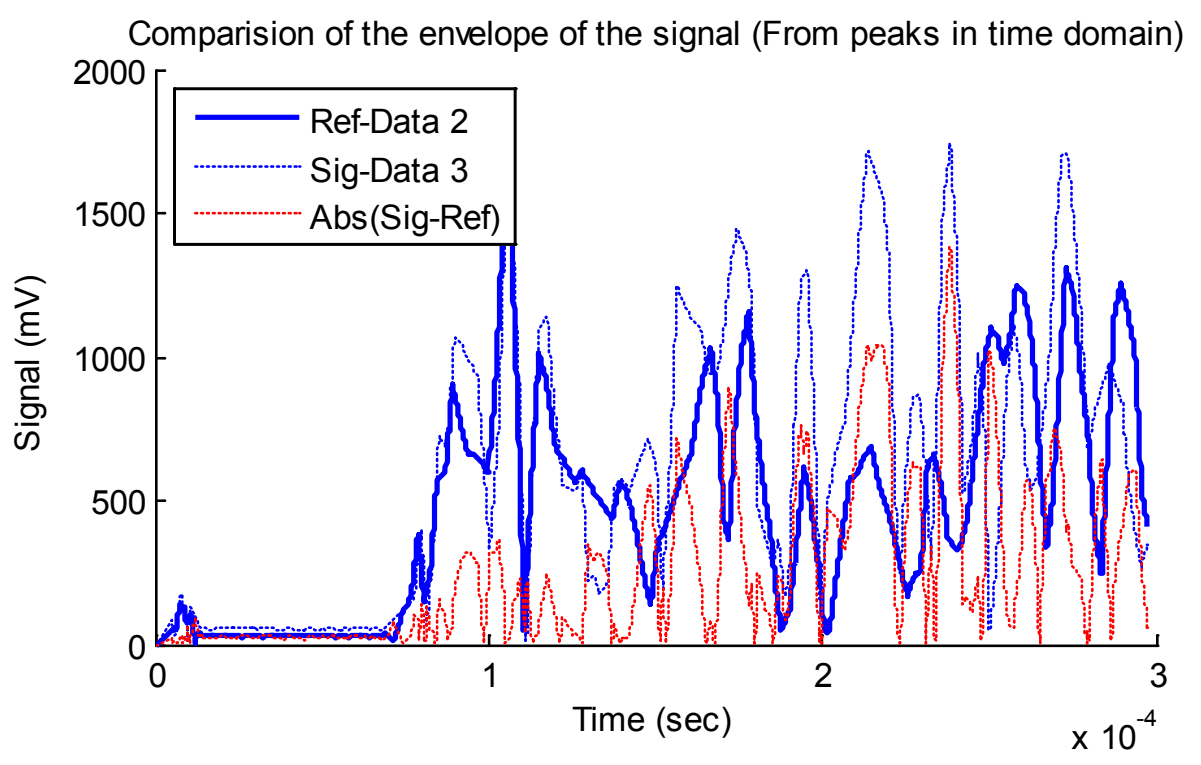

Figure 2.39 - Absolute difference of pipe with first cut (data 2) and pipe with second cut (data 3)

After performing all these calculations the sum of square of difference was calculated among the defect levels keeping the perfect pipe signal as reference. Figure 2.40 shows the bar graph plotted after calculating the sum of square of difference of the two defect levels from the perfect pipe data. It can be clearly visible that the first defect level, which was not a through cut, shows very little change from the reference signal whereas the defect 2 (a through cut) shows a great amount of difference from both the reference and first defect level signal. This graph is of a great importance for real world applications as it indicates the severity of damage occurred to the pipeline 
systems and alerts at first defect level to take safety measures before the defect goes beyond control.

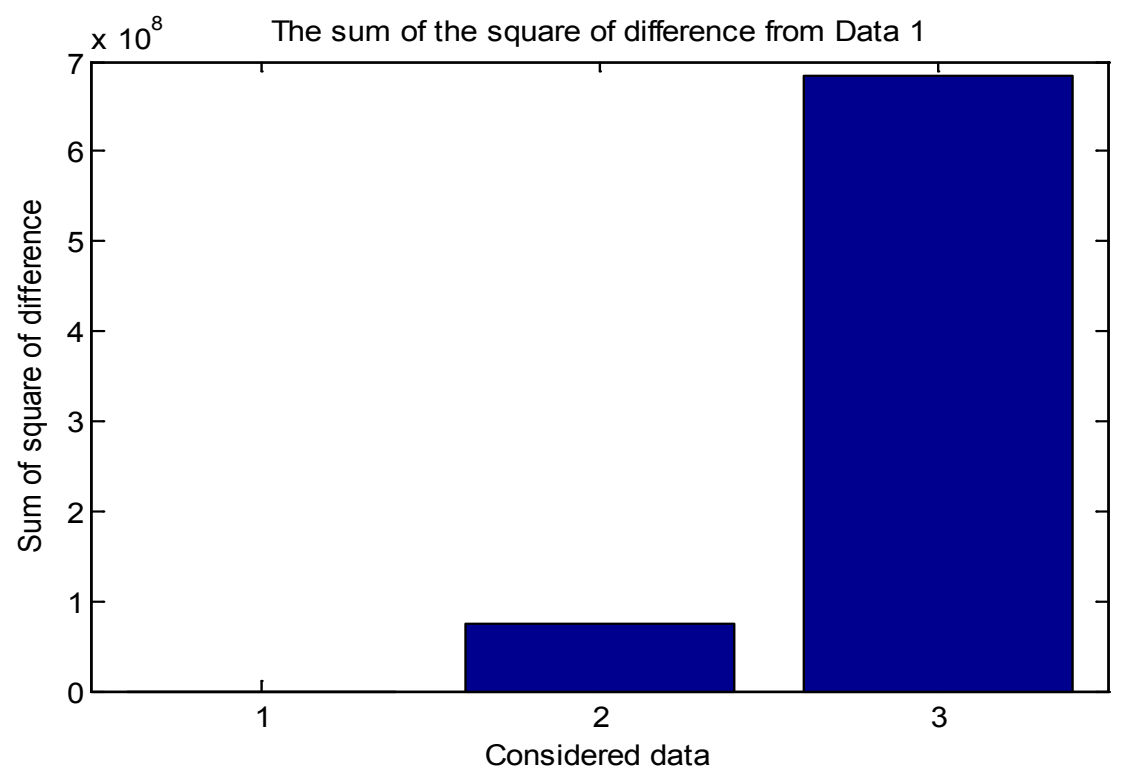

Figure 2.40 - Sum of square of difference of each test case; perfect pipe/reference data (1), small cut (2) and through cut (3)

\subsubsection{Copper pipe with hole at the center}

The pipe with hole (pipe 2) was then excited by the same technique as did for the pipe with cut (pipe1). The piezoelectric element at one side was excited with 3.5 sine waves and the output voltage was recorded at the other end. Matrix plots obtained by exciting the signal are shown in figure 2.41. The signal obtained was in the unprocessed form and almost looks the same for all three cases. The useful information needs to be extracted from the obtained signal to reach any conclusion. The $\mathrm{S}$ - transformation was first calculated to select the excitation frequency for the system. 


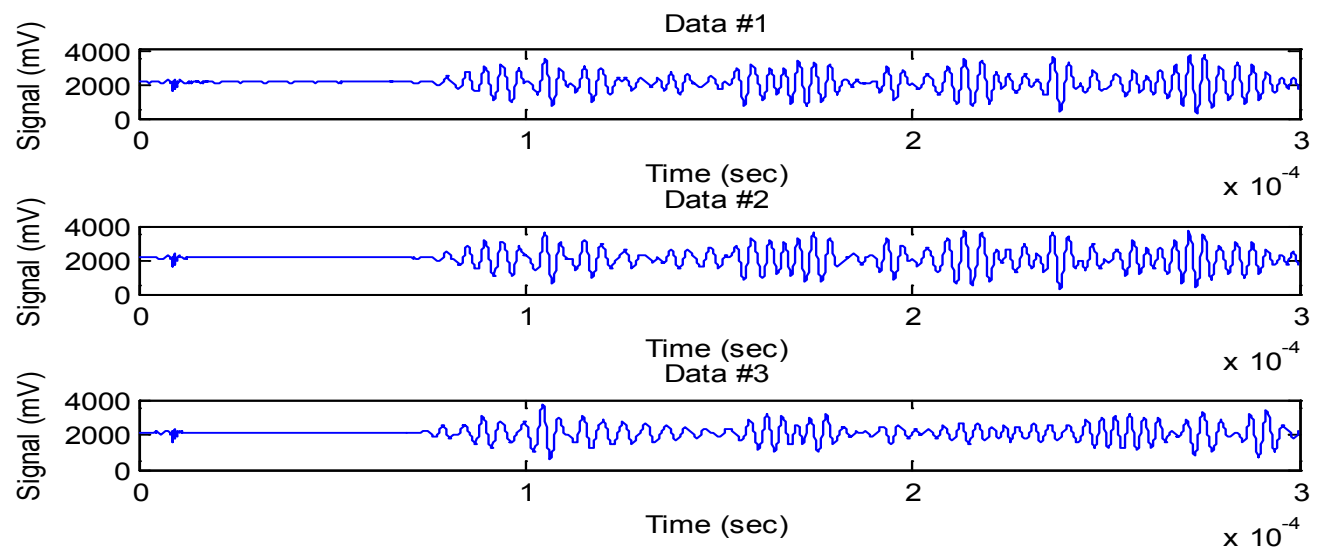

Figure 2.41 - The received signal at sensing piezoelectric element.

The $\mathrm{S}$ - transformation plots for each case is shown in figure 2.42. From the below figures it is noted that the dominant frequency for all the three cases is around $300 \mathrm{KHz}$. The darker area in the plots is the area of interest, rest is either noise or vibration due to the presence of any electric object near the equipment. From the $\mathrm{S}$ - transformations we decided to use $300 \mathrm{KHz}$ as an exciting frequency because of the darker areas present at this particular frequency.

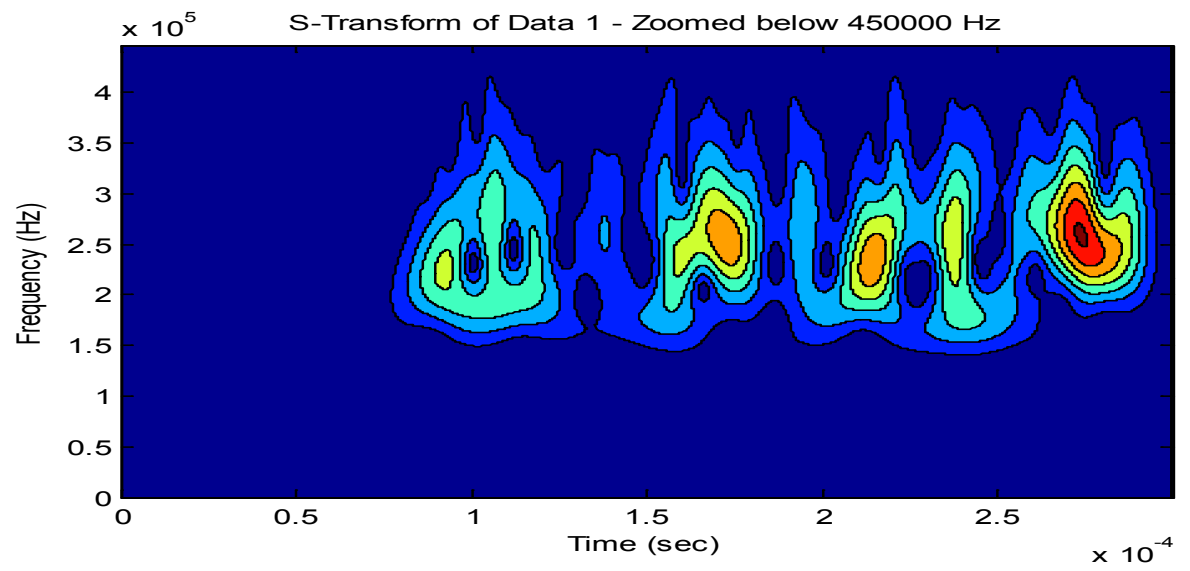

(a) 


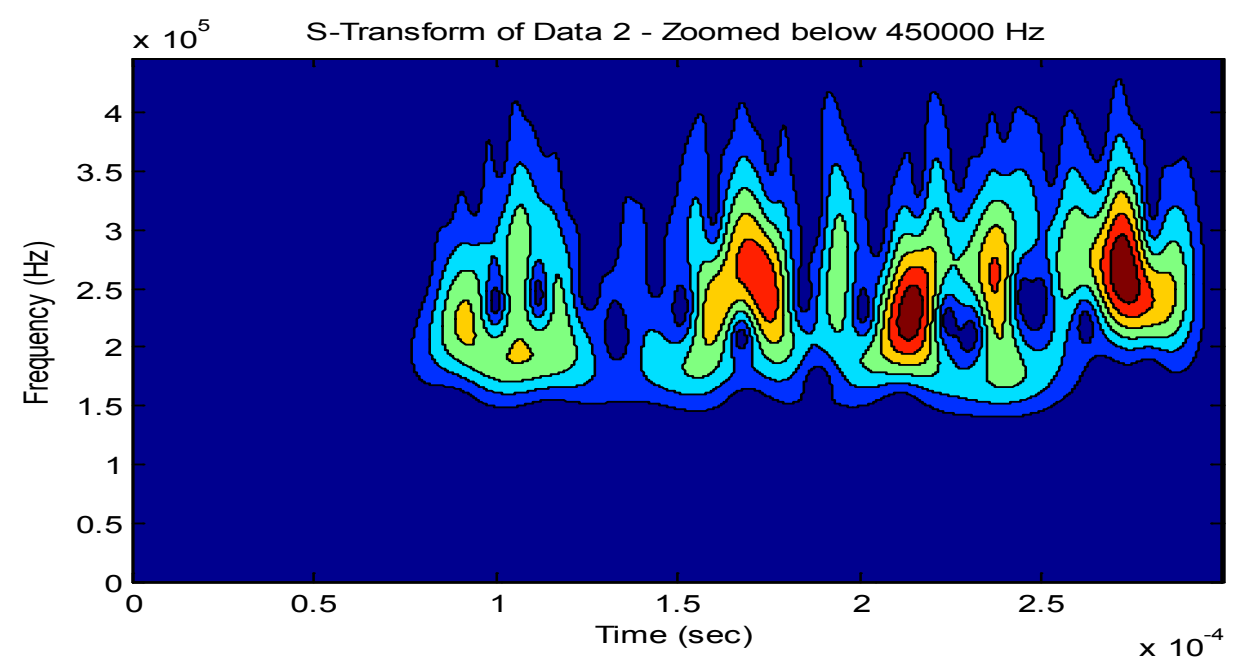

(b)

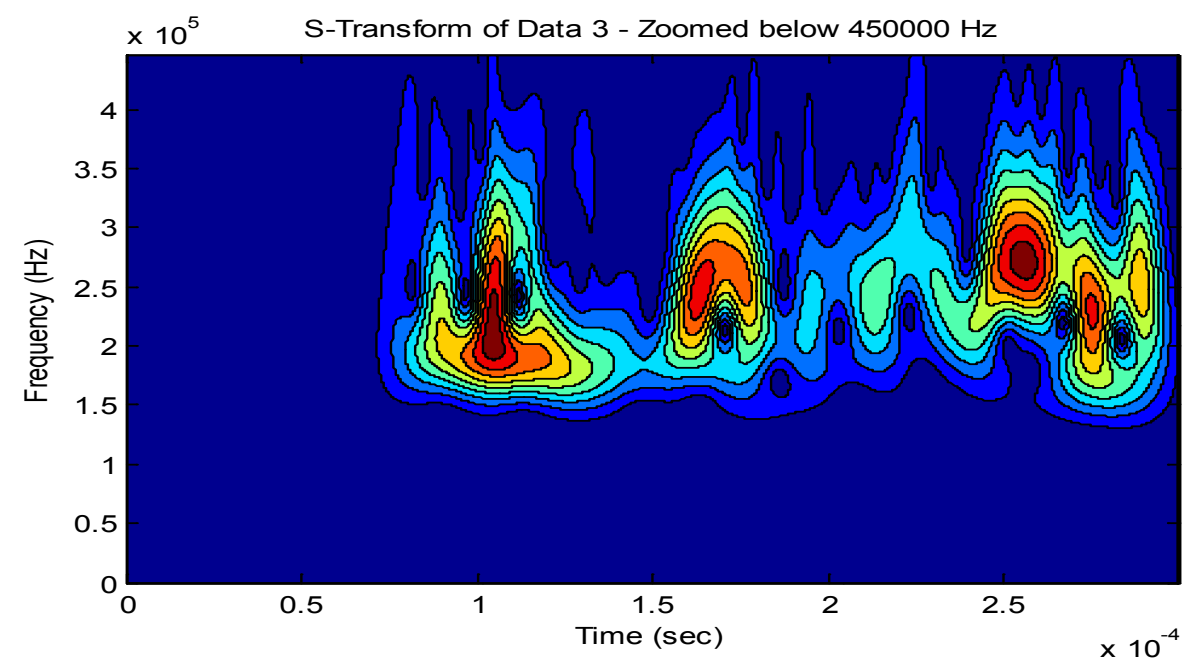

(c)

Figure 2.42 - S-transformation plots below $450 \mathrm{KHz}$ frequency (a) perfect pipe, (b) pipe with small hole and (c) pipe with large hole.

After selecting the excitation frequency, the pipe was excited at 300 $\mathrm{KHz}$ frequency and the signal received was enveloped by $\mathrm{S}$ - transformation and is shown in the figure 2.43. The signals for the perfect pipe and for the pipe with small hole (0.090 inch diameter) move almost on the same path, because the size of the defect was very small. Eventually when the defect size 
was increased ( 0.117 inch diameter hole) the signal changes by a considerable amount, which can be seen in the figure 2.43 clearly. In this type of defect the amplitude of the final signal from the second level defect falls below the other two signals. This means that the amplitude of the envelope of the signal decreases with the increase in the defect size. This case of defect (hole) was opposite to that of the cut as a defect in the pipe.

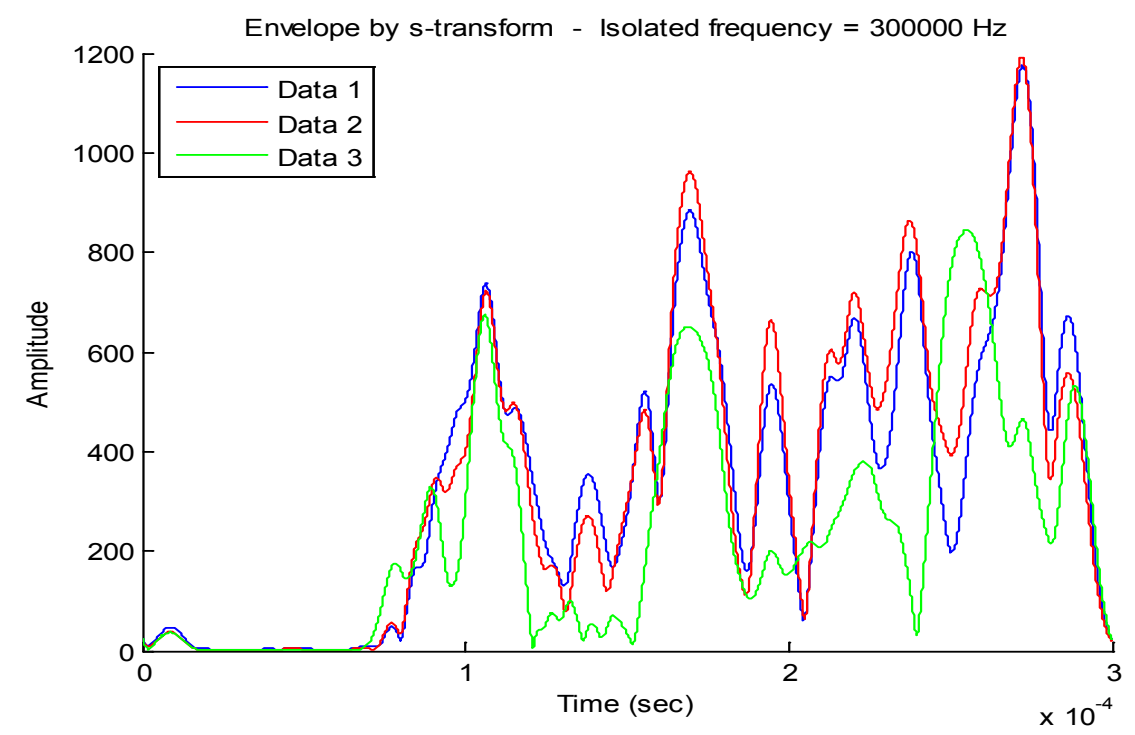

Figure 2.43 - Envelope of the S-transformation of the signal. Perfect pipe (data 1), pipe with small hole (data 2) and large hole (data 3)

The wavelet transformation of the envelope of the original signal and the approximation coefficient of the original signal for all the three cases was calculated and shown in figure 2.44. The absolute error for all the three cases falls below one percent and that too at second level of approximation and the data points for all the cases came down to 150 from 600 . The absolute errors are $0.34,0.38$ and 0.33 percent for case 1 , case 2 and case 13 respectively. The 
data points decreased by consequential amount, which in real applications can be of great use and could, save a considerable amount processing time.

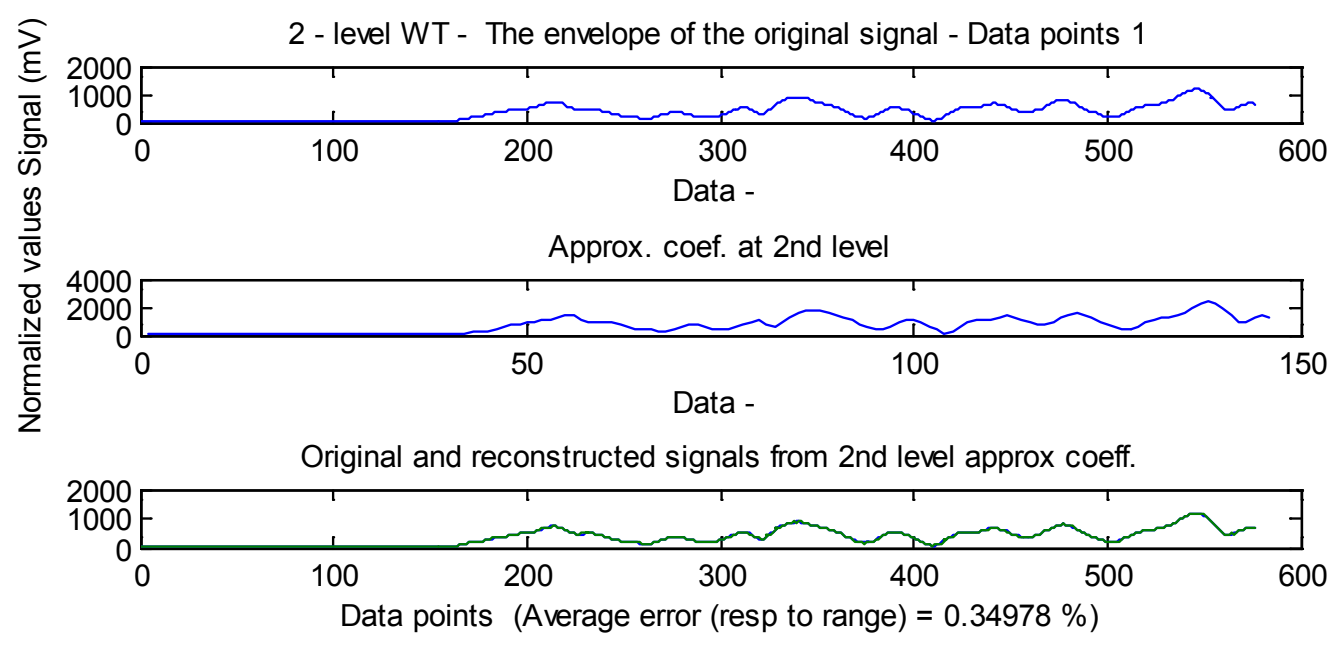

(a)

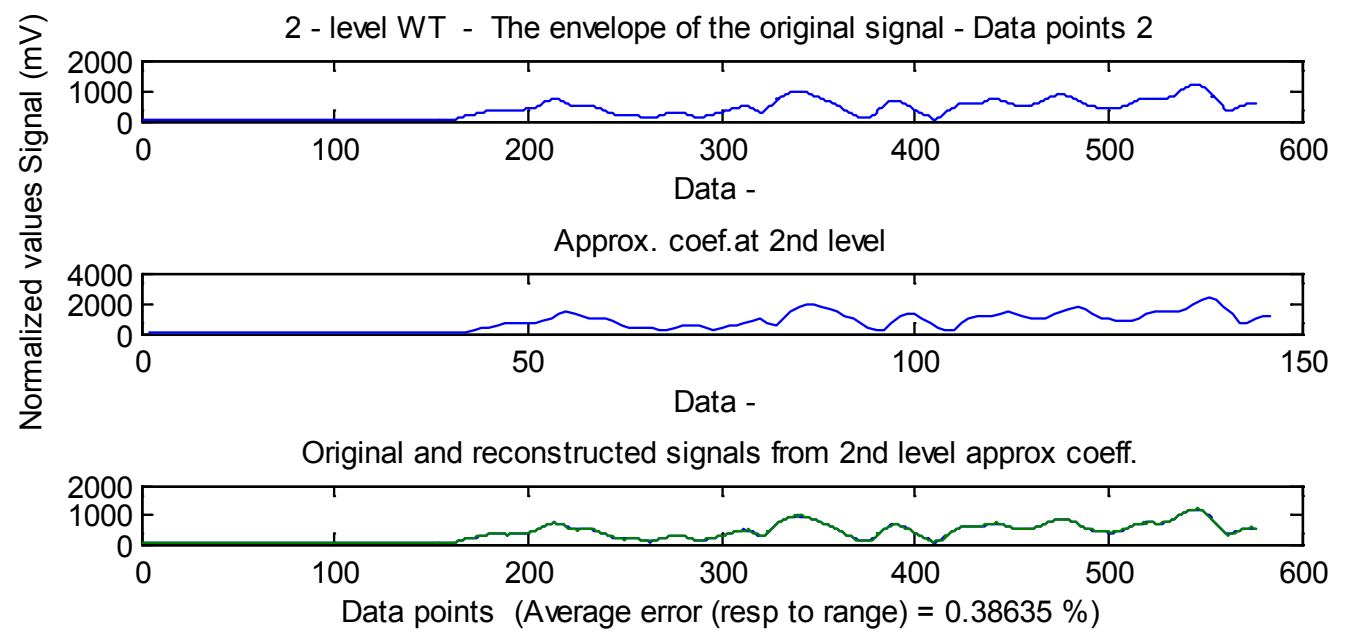

(b) 


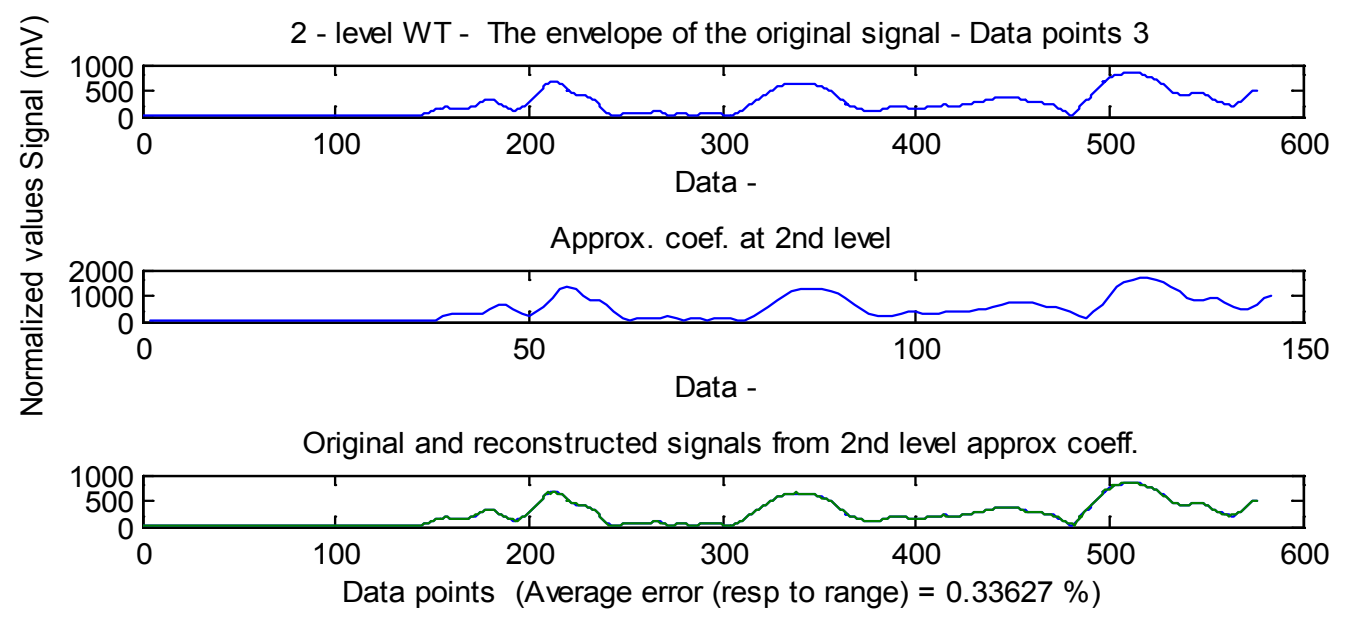

(c)

Figure 2.44 - Wavelet transformation of the original signal, second level approximation of the envelope and average error; from (a) perfect pipe, (b) small hole at center and (c) large hole at center.

Comparison of the envelope of each set of data in time domain for three set of data is shown in figure 2.45. The results came out to similar to that of the pipe with cut. The difference for data 2 (small hole) and data 1 (perfect pipe) is lower than that of between data 1 and data 3 (bigger hole).

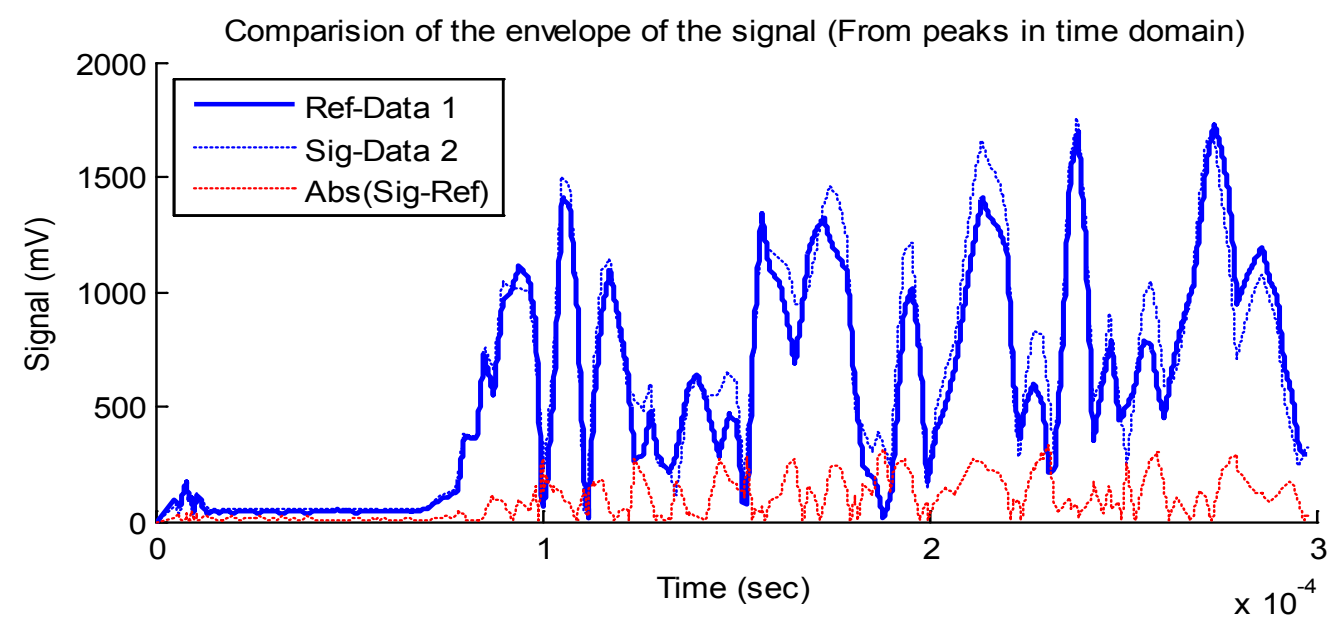

(a) 


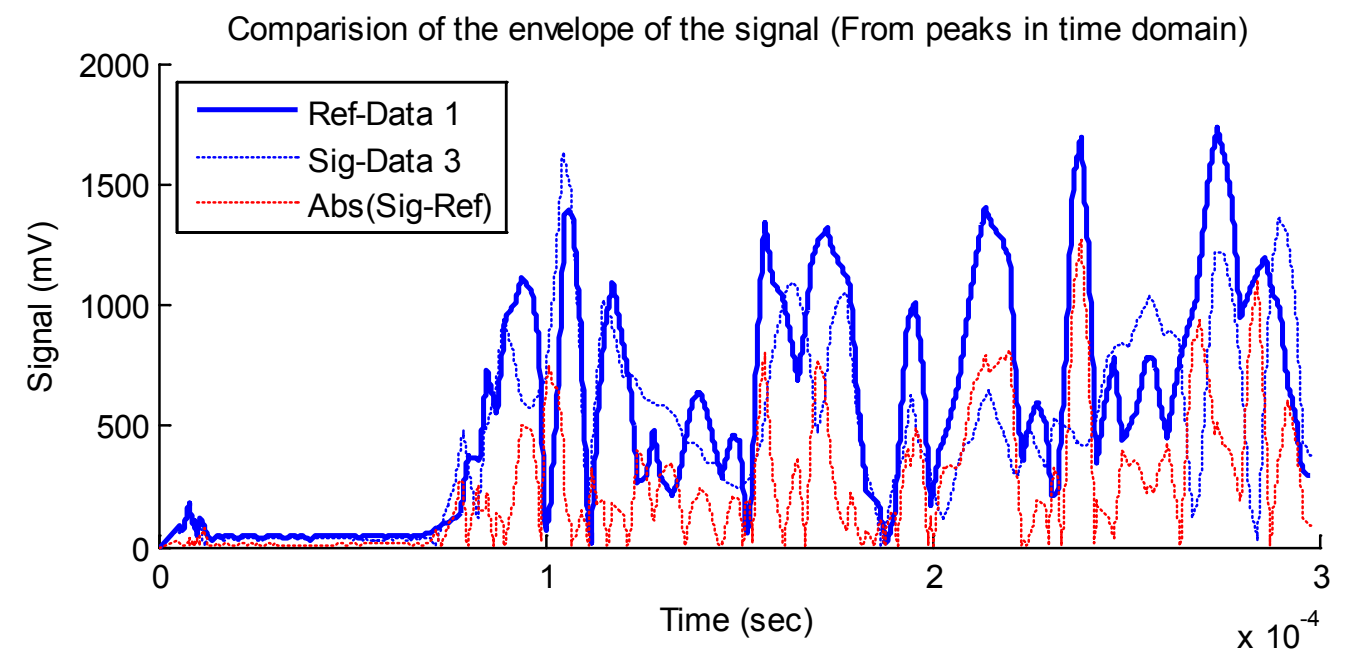

(b)

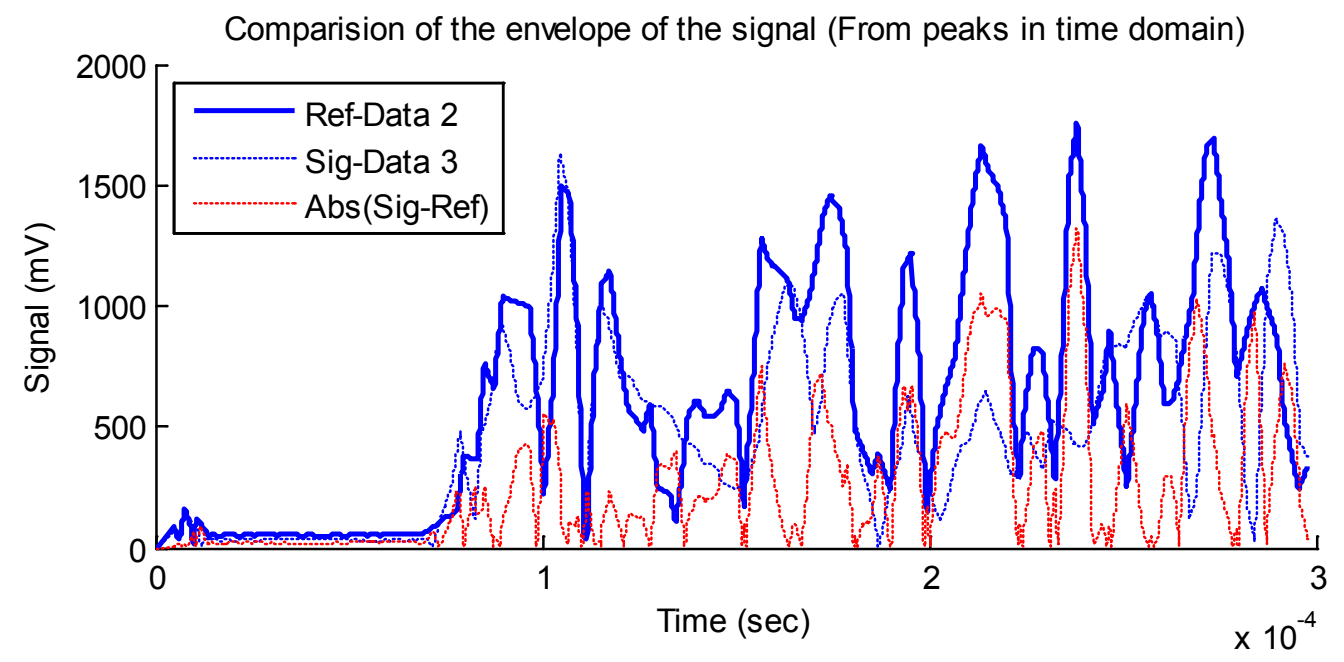

(c)

Figure 2.45 - Absolute difference of the envelope of signal; (a) perfect pipe (data 1) and pipe with small hole(data 2), (b) perfect pipe(data 1) and pipe with large hole(data 3) and (c) pipe with small hole(data 2) and pipe with large hole(data 2)

The sum of square of difference from the reference data (perfect pipe) is calculated at the end and is shown in figure 2.46. The difference in bar height is convincible and can be seen clearly from the figure. The square of difference between the reference signal and the signal from bigger hole (data 
1) has a huge amount of difference which is clearly visible as compared with the difference between the reference signal and the signal from the smaller hole (data 2).

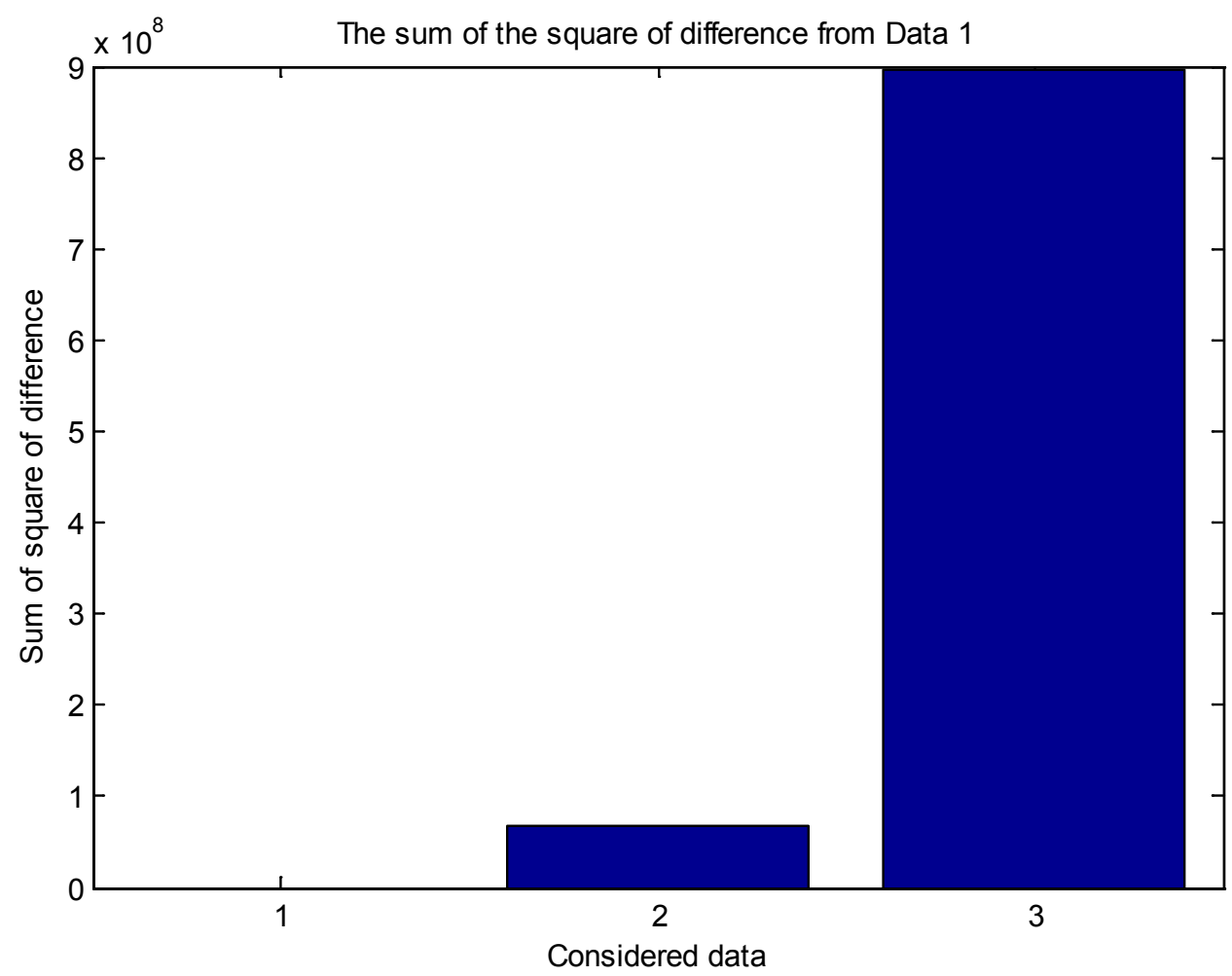

Figure 2.46 - Sum of square of difference of each test case; perfect pipe/reference data(1), small hole(2) and large hole(3)

\subsubsection{Copper pipe with dent at the center}

A 12 inch copper pipe with perfect condition and at two dent levels was excited with lamb wave approach from one end at $300 \mathrm{KHz}$ and signal was received at the other end. The matrix plot from three test cases is shown in figure 2.47. The signals look very similar to each other except at $200 \mu \mathrm{sec}$, the difference can be seen among the signals. 


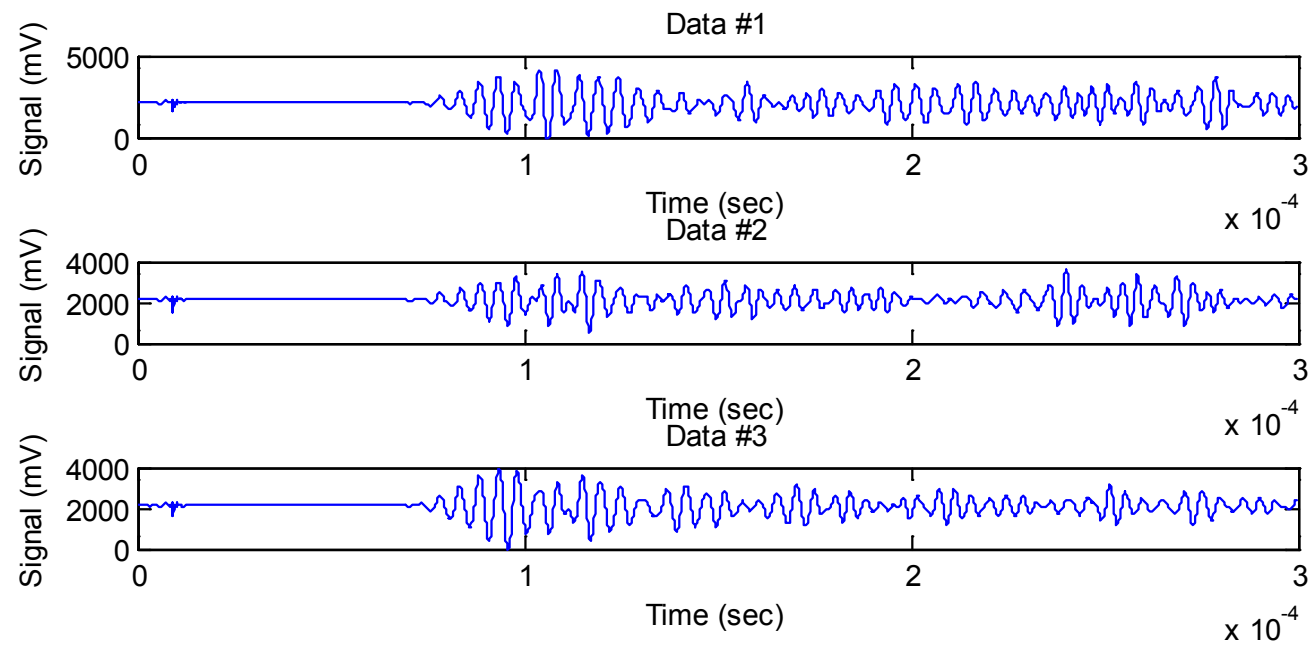

Figure 2.47 - The received signal at sensing piezoelectric element.

The $\mathrm{S}$ - transformation calculated for the received signal is shown in figure2.48. The response of the signal was received between $150 \mathrm{KHz}$ to 300 $\mathrm{KHz}$ at approximately100 $\mu$ sec. At rest of the time interval the traces received were due to the disturbance from electric and mechanical equipments.

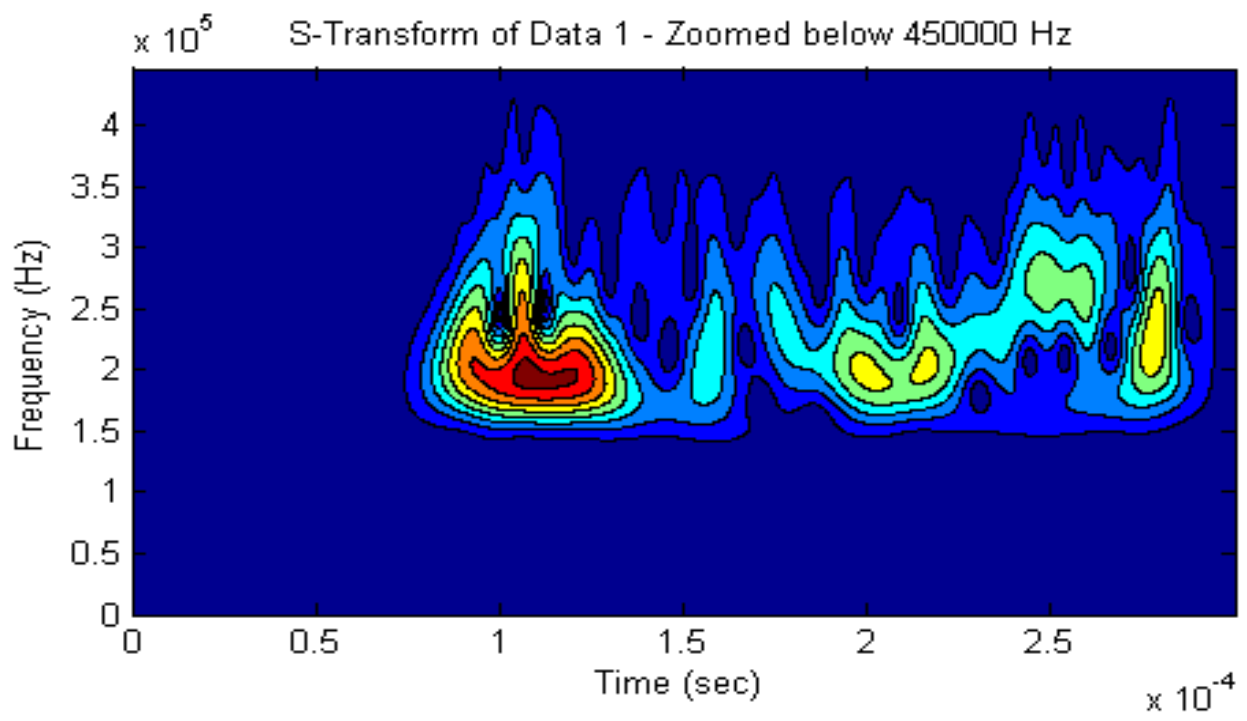

Figure 2.48 - S-transformation plot, zoomed below $450 \mathrm{KHz}$ frequency for perfect pipe 
The envelope by $\mathrm{S}$ - transformation at isolated frequency of $300 \mathrm{KHz}$ was calculated and plotted as shown in figure 2.49. The signals came out to be very unsymmetrical and are not clear as can be seen in the figure. The graphs from all three data sets at $100 \mu$ sec show some meaningful information. After $100 \mu$ sec time frame the spectrum becomes absurd and no indicative information can be drawn out of that spectrum.

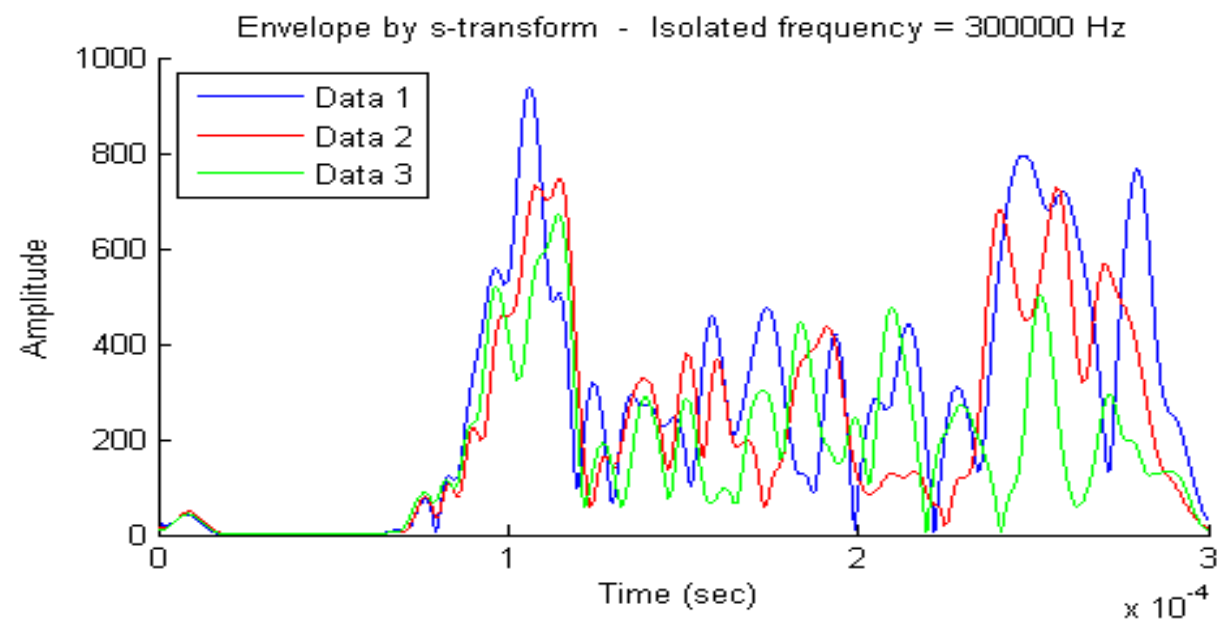

Figure 2.49 - Envelope of the S-transformation of the signal. Perfect pipe (data 1), pipe with small dent(data 2) and large dent(data 3)

The intensity of defect was then calculated followed by the approximation of the original signal. The intensity of the defect shown in figure 2.50; is plotted by calculating the sum of square of the difference of the defect levels from the reference signal (perfect pipe). It is undoubtedly seen from the bar graphs that with increase in the defect level the amplitude of the bars rises as well. 


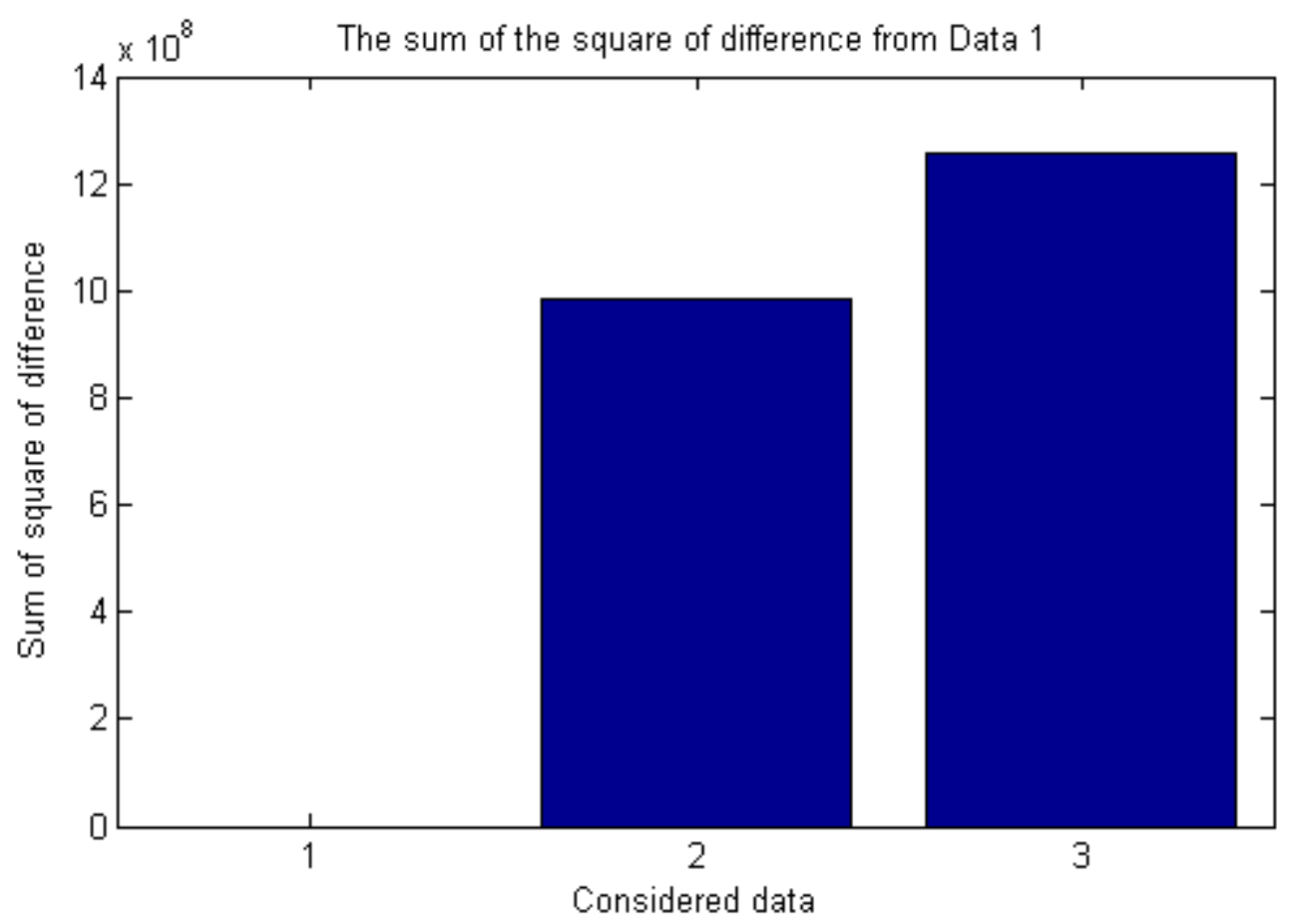

Figure 2.50 - Sum of square of difference of each test case; perfect pipe/reference data (1), small dent (2) and large dent (3)

To save the time of processing of the received data, the number of data points has to be reduced but without affecting the information carried by the signal. The approximation of the original signal was calculated by keeping the absolute error below the acceptable value and can be seen in the figure 2.51,figure 2.52 andfigure 2.53 for perfect pipe, pipe with first defect level and for the second defect level respectively. 


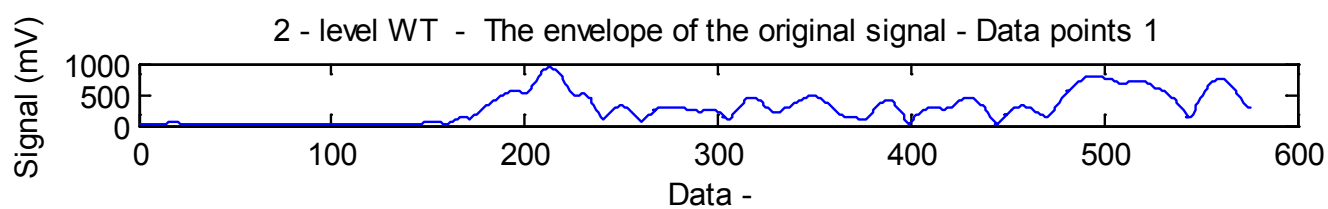

Approx. coef. at 2nd level

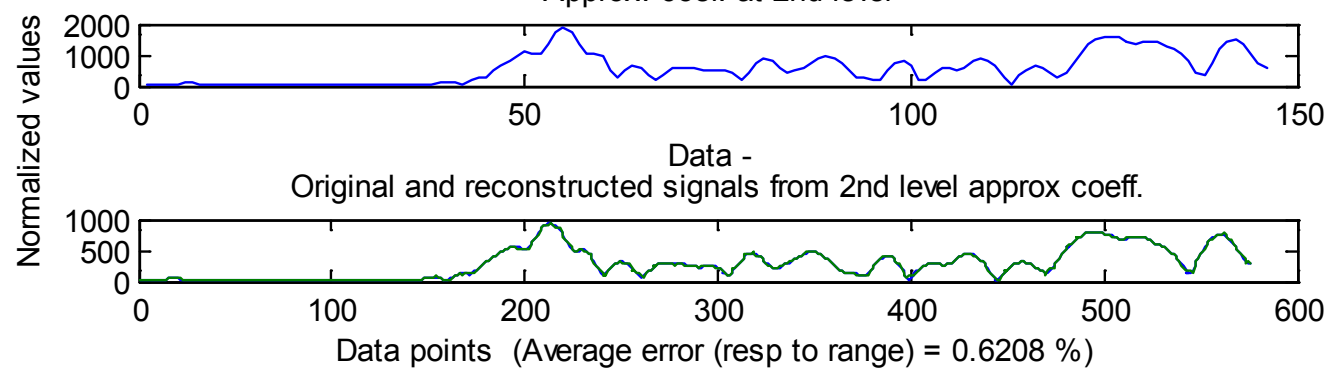

Figure 2.51 - Wavelet transformation of the original signal from perfect pipe, its second level approximation and average error.

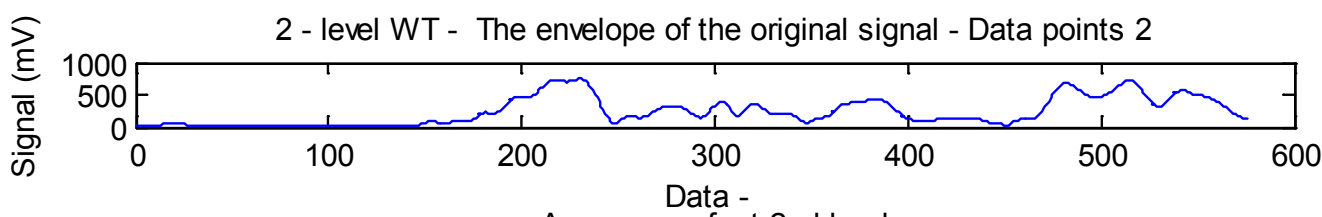

Approx. coef. at 2nd level

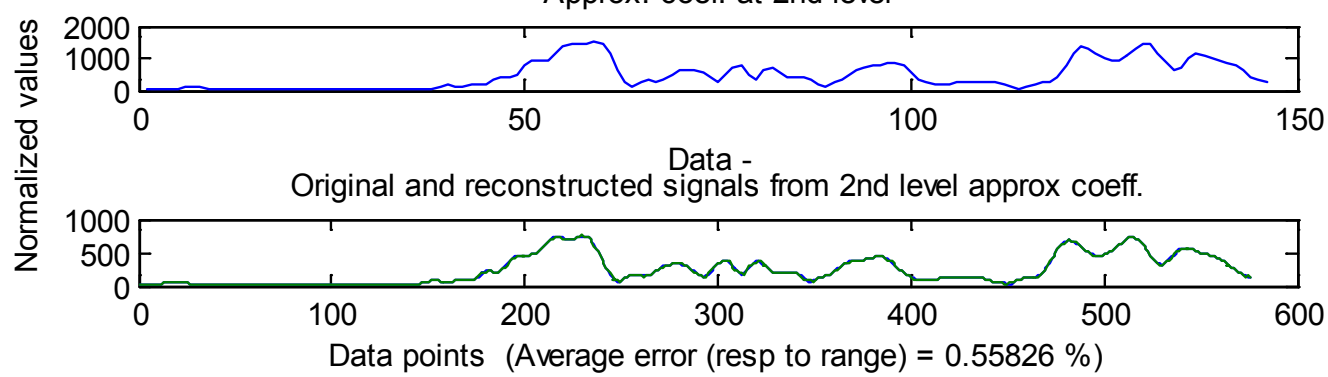

Figure 2.52 - Wavelet transformation of the original signal from small dent, its second level approximation and average error. 


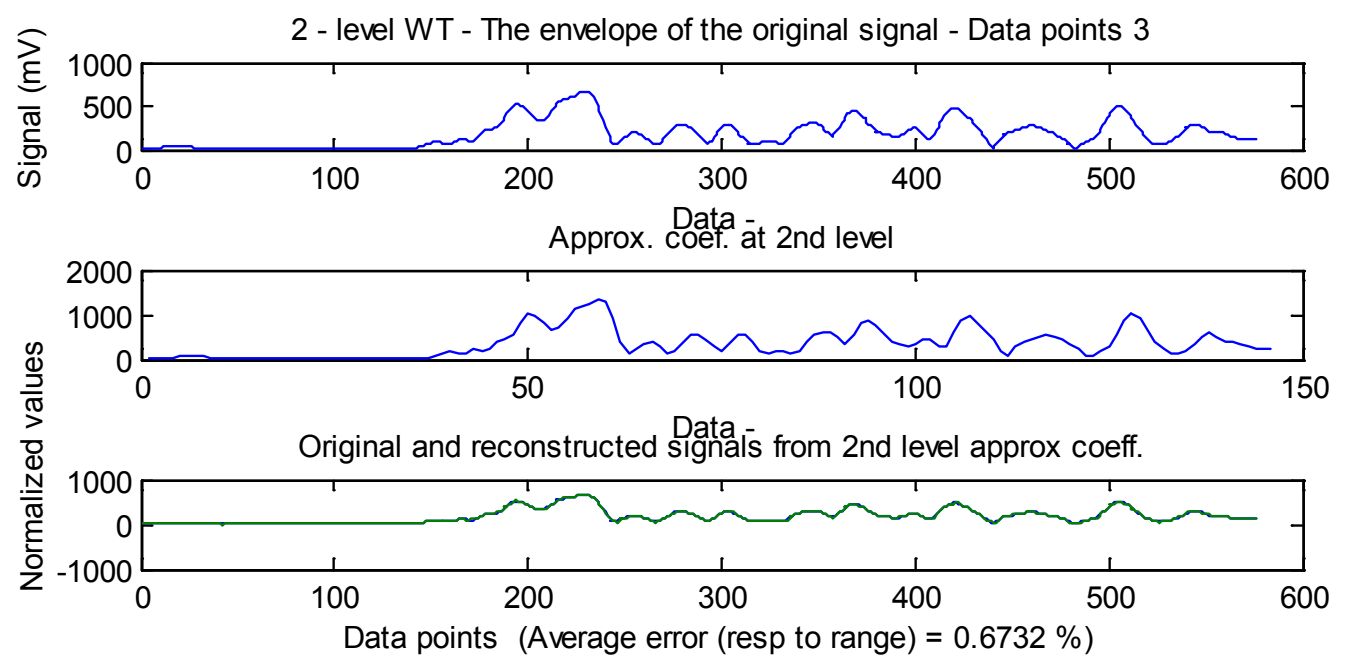

Figure 2.53 - Wavelet transformation of the original signal from large dent, its second level approximation and average error.

\subsubsection{Comparison of three defects}

After discussing the three types of defects (cut, hole and dent) individually, the comparison between the three cases was then determined. The extreme defect levels were considered for comparing thesignals with the reference signal (perfect pipe). The intensity levels of the three defects were calculated and can be seen in figure 2.54. The pipe with through cut defect is considered to be the ardent defect of all the three cases followed by the pipe with hole and pipe with dent.The cut in the pipe is the most severe defect as depicted from the figure below. The dent would be considered to be the minor of all the three defects. 


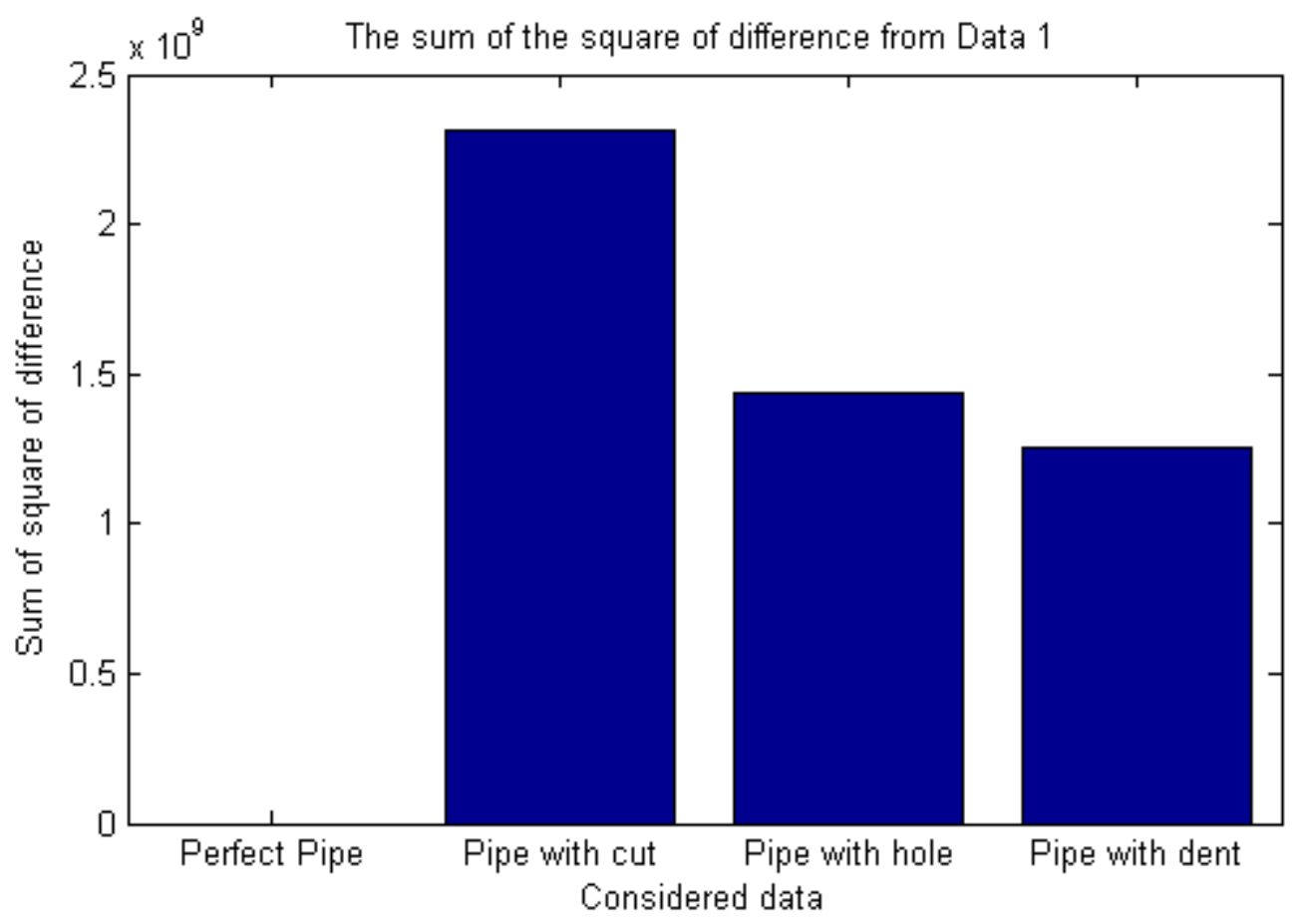

Figure 2.54-Sum of square of difference of each test case and perfect pipe/reference data

\subsubsection{Determine attenuation of signal over long distance}

For performing the above experiments, the length of the copper pipe used was not very long because in those experiments our main motive was to find the behavior of different kinds of defects and also the comparison of all the defects together. That is why we kept the size of the pipe 12 inch rather than to use a very long pipe. As the distance between the sensors was close the strength of the signal was never be an issue for the above set of experiments.

To see the difference when the distance between the sensors increases, a long copper pipe (120 inch) was used with three sensors. Two sensors were 
glued at 1 inch from both the ends and third one was placed at the center of the pipe. One of the piezoelectric elements present at one of the end was used as the exciter and the other two as receivers. A piezoelectric element was excited with continuous sine wave at $200 \mathrm{KHz}$ generated by signal generator. The experimental setup for this experiment is shown in figure 2.55. A BK Precision, $10 \mathrm{MHz}$ signal analyzer was used to excite the sensor with continuous sine wave. The signal was amplified by TEGAM power amplifier, model 2348 before exciting the piezoelectric sensor and the reception of the signal was collected by four channel Tektronix TDS 2024B digital oscilloscope.

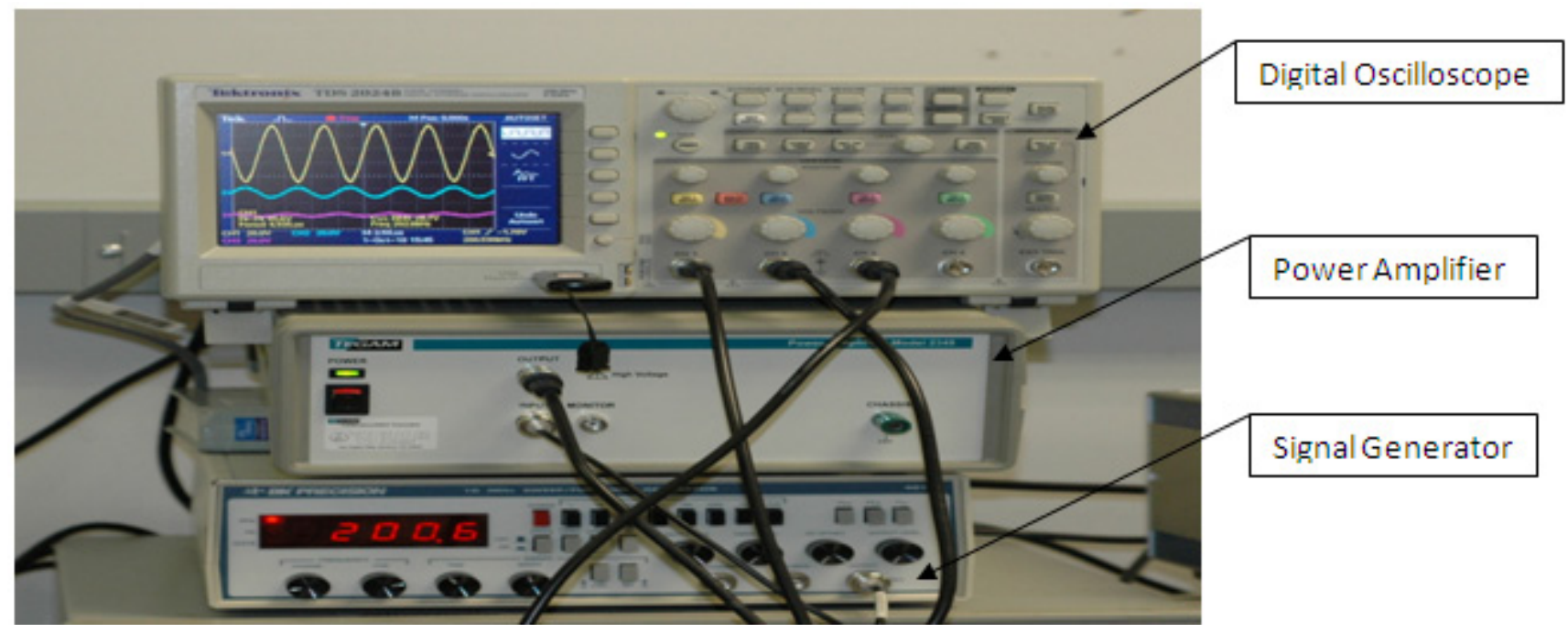

Figure 2.55 - Experimental setup for determining the attenuation of signal over long distance

The sine wave used for excitation was first amplified by using power amplifier before exciting the piezoelectric element. The output voltage was recorded by digital oscilloscope and can be seen in figure 2.56 . The amplitude 
of the exciter is largest and keeps on decreasing for the successive piezoelectric element. This shows that the signal attenuates as the distance between the piezoelectric elements is increased. We are getting the signal from all the piezoelectric elements but the strength of the signals becomes very low that they cannot be used to perform further calculations and get some substantial information out of the received signal. So, in order to maintain the strength of the signal throughout the length of the pipe, either an array of sensors close to each other should be used or the input signal has to be amplified enough which can travel a long distance without compromising the strength of the signal. The signal for the piezoelectric element attached at the end of the pipe is seems to be almost dead having very little amplitude whereas the center piezoelectric element still holds some amplitude and is visible in the figure below. 

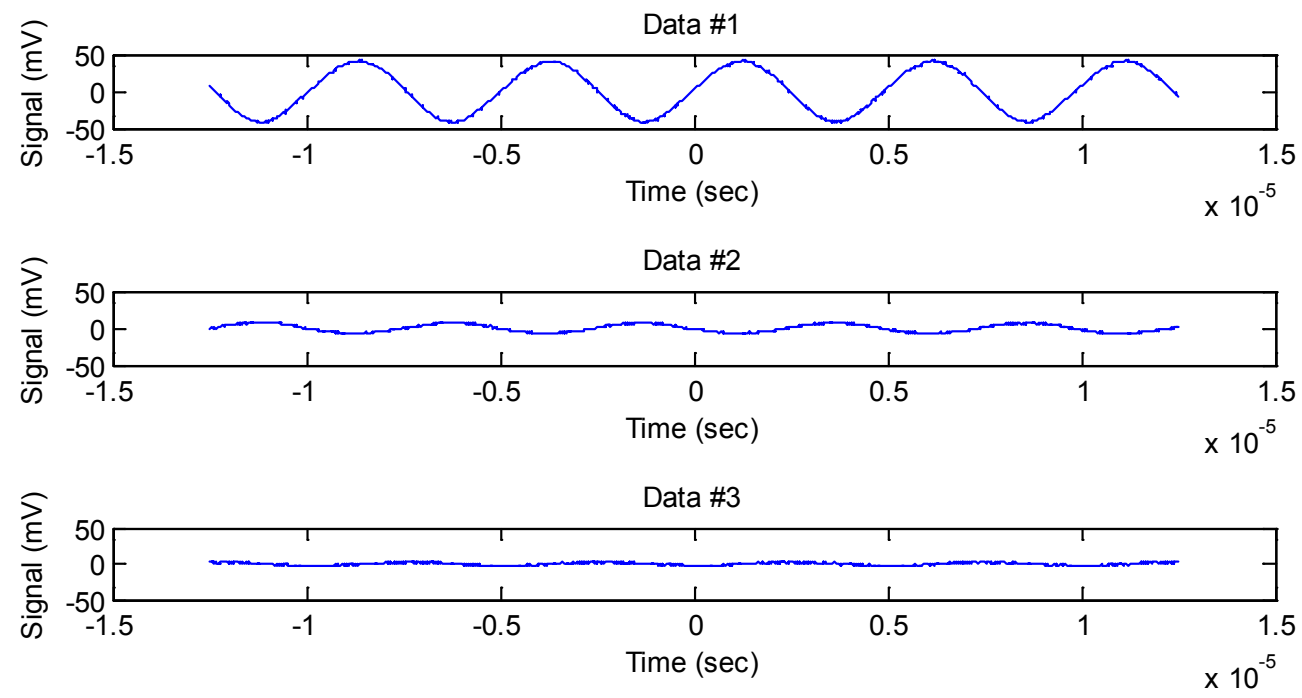

Figure 2.56 - Showing strength of signal at exciter (data1), middle sensor (data2) and sensor at the end of long copper pipe (data3)

\subsubsection{Health monitoring of Composite Tubes against defects}

Carbon fiber tubes (composite tubes) are being used in various industrial segments including the oil and gas industry. Carbon fiber tubes are now replacing the metal tubes including the titanium because of very light weight and low coefficient of thermal expansion. Tubes made out of carbon fiber are more corrosion resistant than the metals and has a low heat transfer rate. The carbon fiber tubes are being used in the mosquito air helicopters because they are more durable, tough and affordable [59].

Three carbon tubes of each 7.5 inch length, 0.2 inch outer diameter and 0.05 inch thickness were used to perform the experiments by sweep sine wave and lamb wave approach. Three tubes were used to perform the experiments, 
namely perfect tube, tube with 1 inch notch at the center of the tube and a through hole at center.

Two piezoelectric elements were glued to each tube at both the ends. The signal analyzer was used to excite one of the piezoelectric elements with sweep sine wave and frequency response was collected from the other piezoelectric element by the same signal analyzer. The frequency domain signal obtained from three cases is shown in figure 2.57. Nothing can be predicted from the signal obtained by the signal analyzer. The square of difference between the signals of the defected tubes and perfect tube was plotted and can be seen in the figure 2.58. The difference between the two spectrums is nearly the same except at $75 \mathrm{KHz}$ to $85 \mathrm{KHz}$ of frequency range.

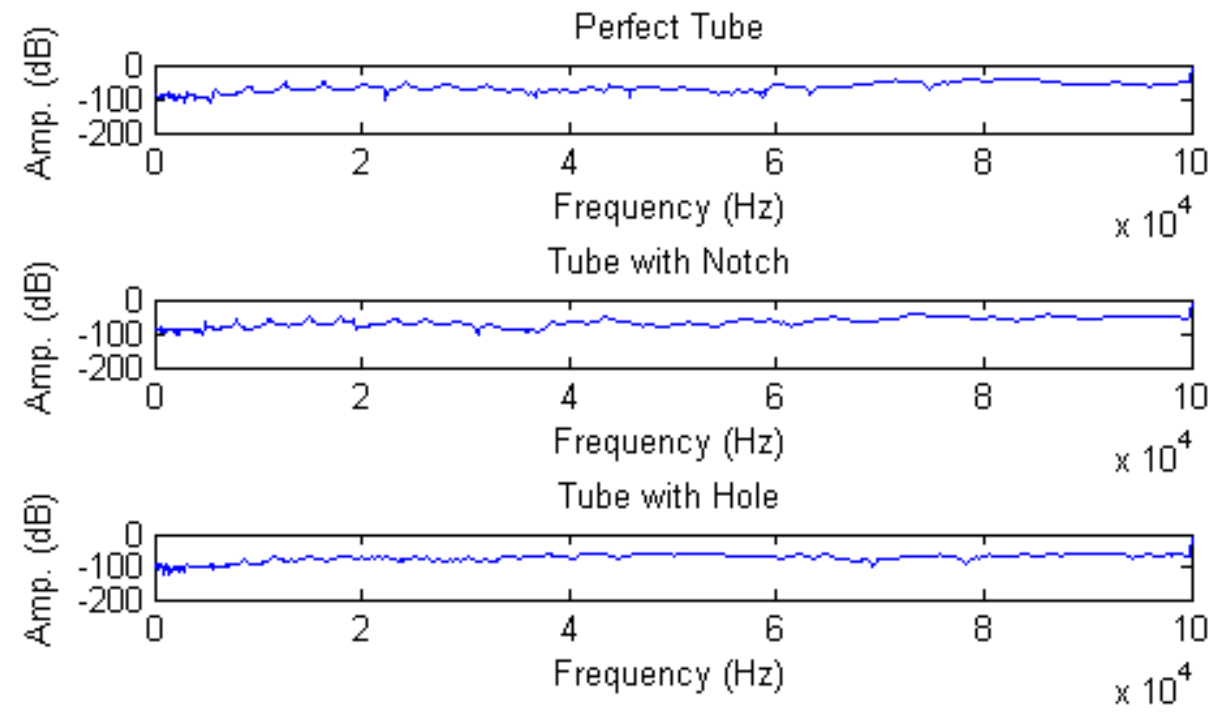

Figure 2.57 - Spectrum analysis of perfect tube, tube with notch and through hole. 

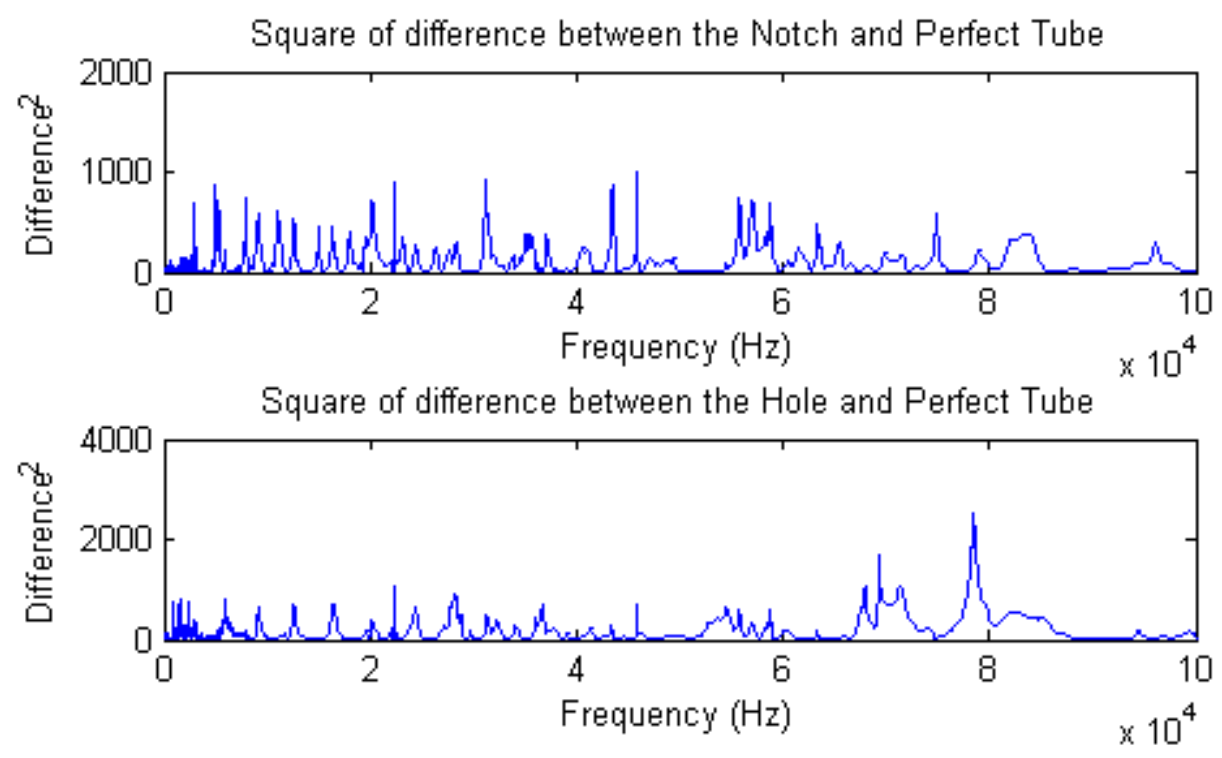

Figure 2.58 - Square of difference between perfect carbon tube and tubes with notch and center hole.

The equipment used for lamb wave approach was the same as was used for performing copper pipe experiments. The matrix plot for the three carbon tubes is shown in figure 2.59. The difference between the signals is easily spotted and further processing is needed to clarify the difference among the signals. $\mathrm{S}$ - transformation of the signals was calculated in order to isolate the noise coming in the signal. The $\mathrm{s}-$ transformation plot is shown in figure 2.60 . 

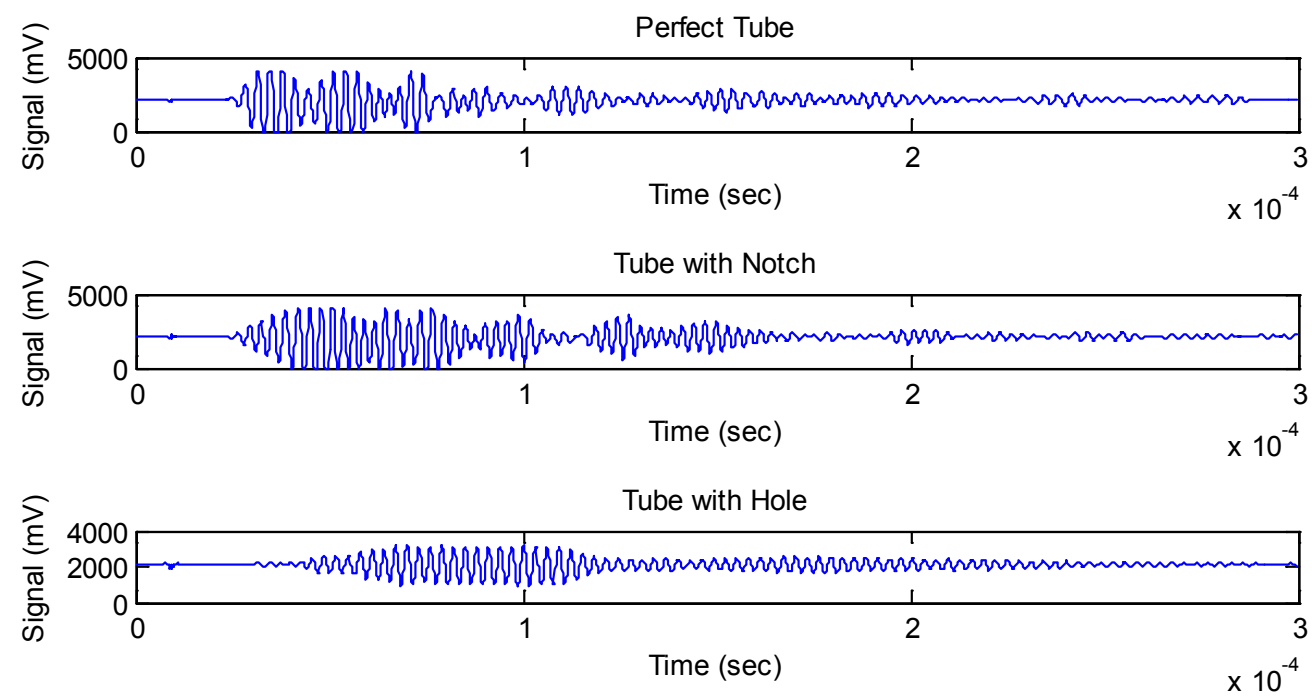

Figure 2.59 - The received signal at sensing piezoelectric element.

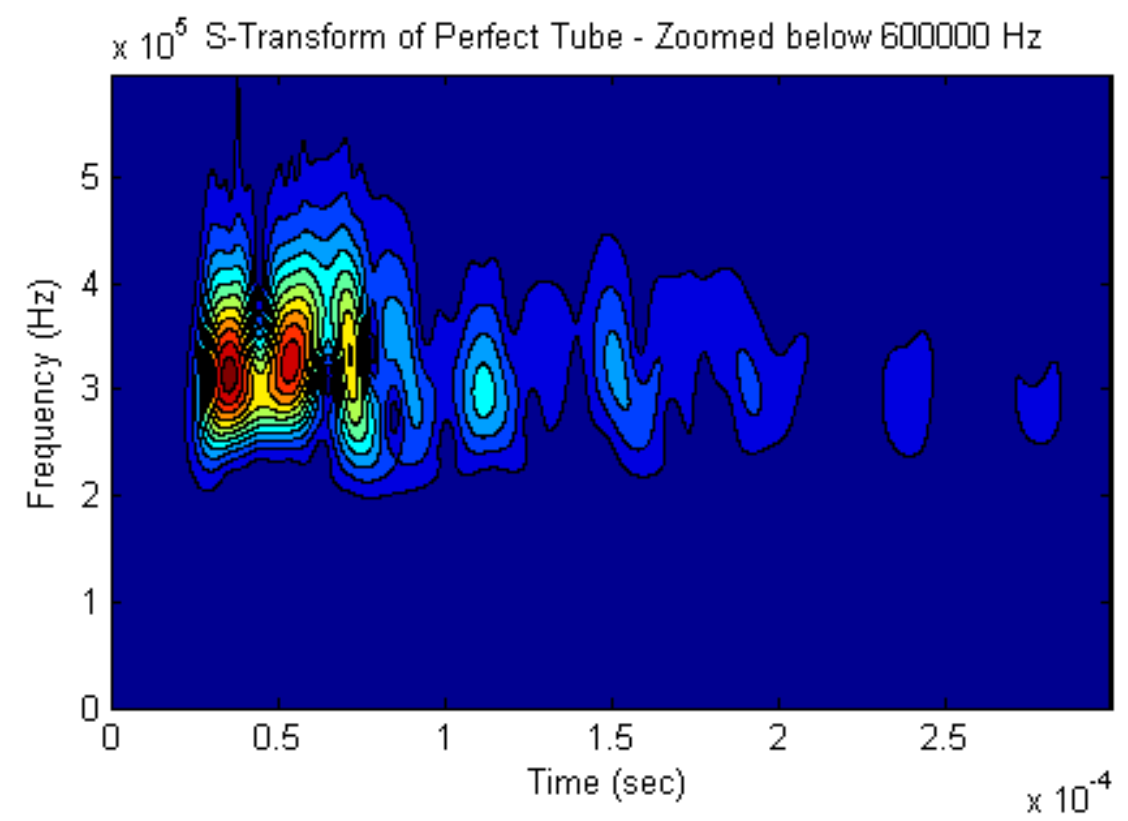

Figure 2.60 - S-transformation plot, zoomed below $600 \mathrm{KHz}$ frequency for perfect pipe

As the echoes from the signal are present around $300 \mathrm{KHz}$ frequency, the envelope of $\mathrm{S}$ - transformation was calculated at $300 \mathrm{KHz}$ frequency and 
is shown in figure 2.61. Figure shows that the amplitude of the perfect tube is the highest followed by the tube with notch and hole. The scattering of the signal from the point of defect could be reason for the decrease in the amplitude. The defect may act as the barrier for the signal to reach the other side with the same strength. The results for the envelope of the signal on carbon tubes are opposite to that obtained from the copper pipes.

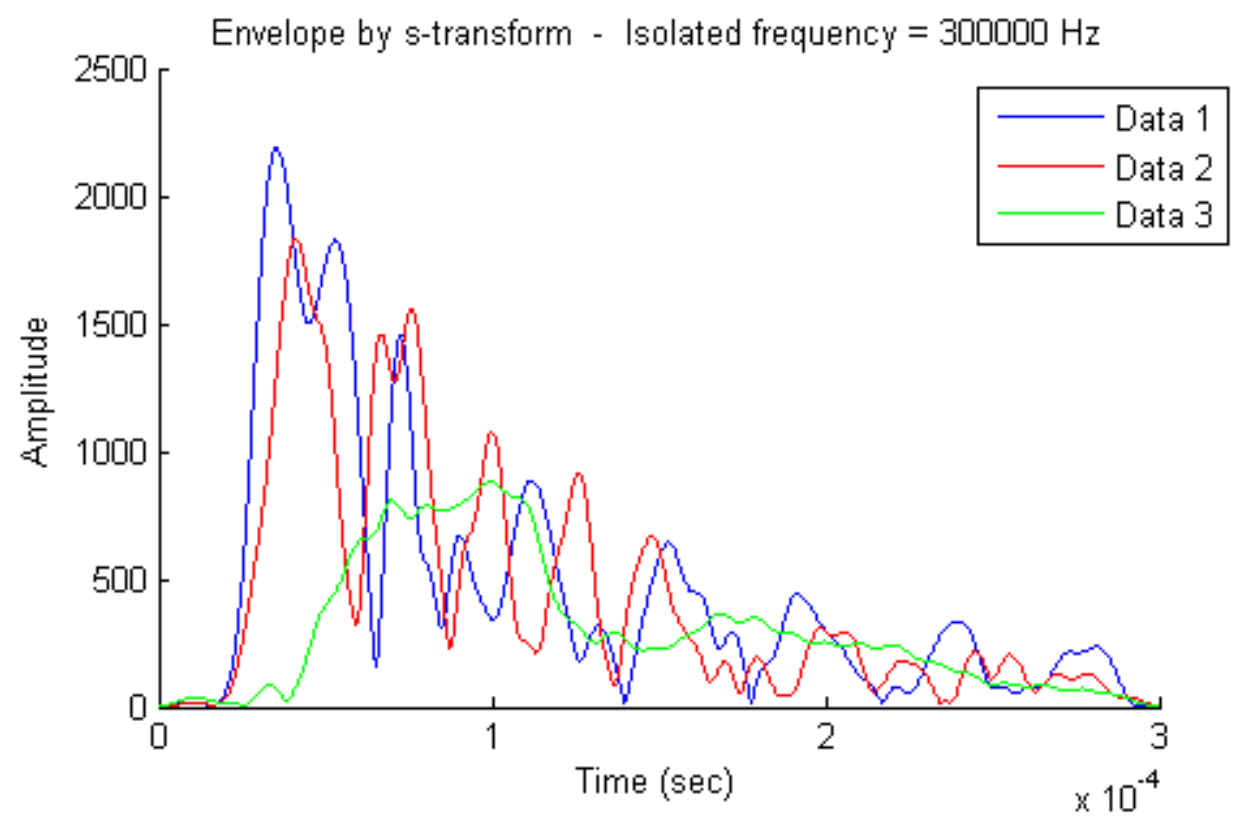

Figure 2.61 - Envelope of the S-transformation of the signal. Perfect tube (data 1), tube with notch (data 2) and tube with hole (data 3 )

Finally the intensity of the defects was calculated by taking the sum of square of difference from the reference signal (from perfect tube). Figure 2.62 shows that the defect created by cutting the notch in the tube is more severe than the through hole. The reason can be the defect size, as the size of notch is quite larger than that of the hole. The defect intensity can be seen visually but the motive for calculating it experimentally is to make the detection and 
severity of defect available where the visual inspection is not possible. Hence, the results came out to be convincing and exactly correlates with the actual intensity of defects.

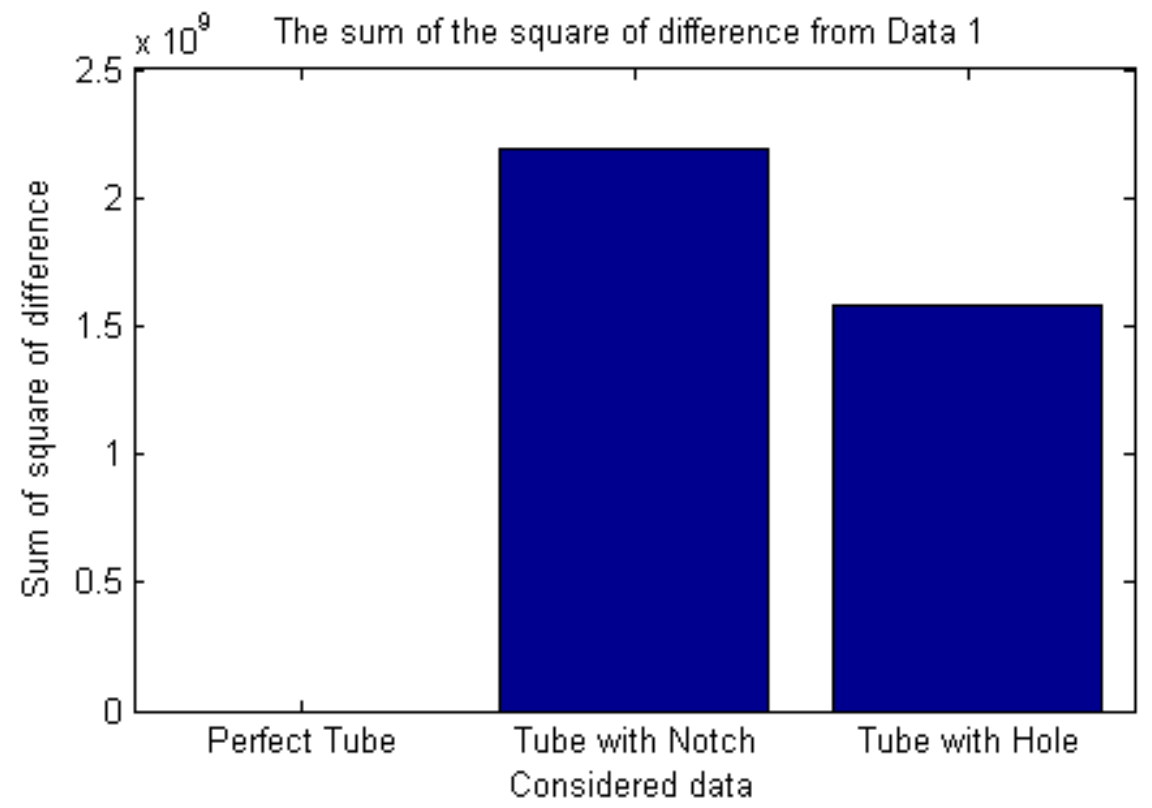

Figure 2.62 - Sum of square of difference of each test case and perfect pipe/reference data

\subsection{Conclusion}

The suitability of the two structural health monitoring approaches was tested and evaluated for different defects present in the copper and composite pipes by using surface waves. The frequency domain spectrum analysis and the time domain lamb wave approach were successfully used to differentiate the perfect pipes from the defected ones. The intensity of defect levels was also produced and verified successfully. Apart from this the propagation and attenuation of the signal in long pipes was also portrayed in this chapter. 
Spectrum analyzer calculated the transfer function of the surface response to sweep sine wave excitation. The results produced by this approach were quite convincing and accurate. Only two piezoelectric elements for every pipe were required to successfully perform the experiments. The cost of the spectrum analyzer was comparatively very low to that of the impedance analyzers, which also work on the same principal (frequency domain analysis). It clearly shows the difference among the intensity of defects present within the system successfully and also depicts the frequency range where the amplitude level differs for different defects.

Lamb waves were successfully generated and received through the hardware used. One piezoelectric element was used to generate the lamb waves and the other one to receive them. The cost of the equipment was the least among the two approaches used in this chapter. The signal gathered from the equipment was transformed into a meaningful information by calculating the envelopes, $\mathrm{S}$ - transformations and wavelet transformations. The $\mathrm{S}$ - transformations provides the clean signal by isolating all the disturbances from the original signal. The intensity of the defects was clearly and accurately calculated by squaring the difference among the signals.

Both of the methods used did a wonderful job in estimating the health of the system as well as determining the severity of defects in them. These 
systems can be considered for structural health monitoring of all plate like structures. 


\section{CHAPTER III}

\section{ESTIMATION OF DRILL WEAR THROUGH SURFACE WAVES}

\subsection{Introduction}

From a long time manufacturing industry has been putting emphasize for tool wear monitoring systems; however, as such consistent commercial and cost effectivetool wear monitoring system has not been developed. Previously, most of the tool wear approximation studies monitored signals coming from the force, acceleration, temperature, acoustic emission and similar source. The complex geometry of the cutting tool and due to very small amount of material loss with the wear of cutting edges makes it difficult to estimate the tool wear through different Structural Health Monitoring (SHM) techniques.

The tool wear can be automatically monitored by using machine vision

systems $[44,45]$ or analyzing the laser beam reflection from the cutting edges [46]. These approaches have not found significant applications in the industry, since the cutting surfaces has to be cleaned properly before each inspection. The cutting forces may be considered as the most informative indirect wear monitoring signal since they are directly correlated with the cutting edges of the tool [47, 48].Most widely used method used for tool wear estimation for the above case was to monitor the spindle current or power measured by the sensor of the machine controller. This process may be automated or further improved by using rotary dynamometers [49 - 51]. 
These methods are still unsuitable for widespread industrial implementations due to the significant amount of cost and limitations for tool wear estimation.

SHM community has been detecting even hard to see damages by simply monitoring the elastic behavior of the surface of the structure. The two approaches developed to monitor the elastic behavior of surface were, impedance characteristics of the piezoelectric transducer attached to the part under observance [52] and the Lamb waves generation and monitoring their propagation by using the same or other piezoelectric transducer [16, 53].

Low cost SHM techniques were developed by measuring the impedance of the single piezoelectric element with an auxiliary circuit and a low cost data acquisition device such as spectrum analyzer [54] or A/D card [55] instead of impedance analyzer. Use of two piezoelectric elements and measurement of the frequency response between them was also purposed [30].

The feasibility of the SHM methods was evaluated in this chapter for monitoring of tool wear. A drill was artificially worn in order to protect the sensors and wires from being damaged and tested by using the SHM methods. The complexity of the tool geometry and distant location of the sensors from the cutting edges were two important challenges need to be 
considered. In the following section differentiation of the measured characteristics and $\mathrm{S}$ - transformation will be briefly introduced.

\subsection{Experimental Setup}

A high speed steel (HSS) drill having 19/64 inch diameter and 4.4 inches in length was used. Two round piezoelectric elements of 0.25 inch diameter and 0.020 inch thickness were bonded (M-Bond 200) to the shank of the drill bit. The location of the piezoelectric elements and the data collection equipment are shown in figure 3.1 .

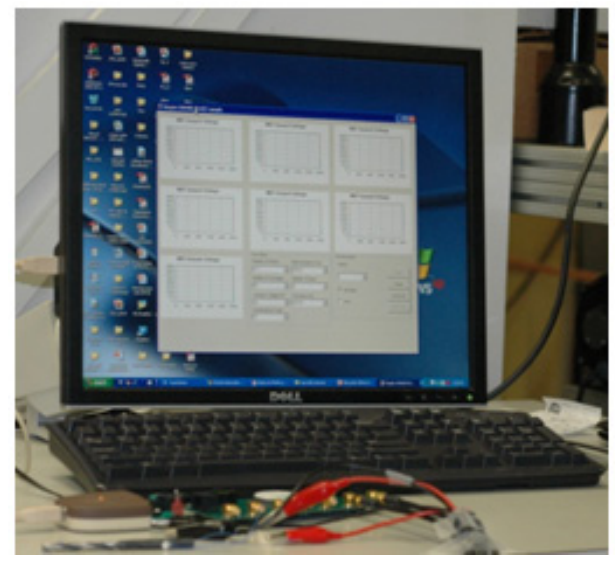

(a)

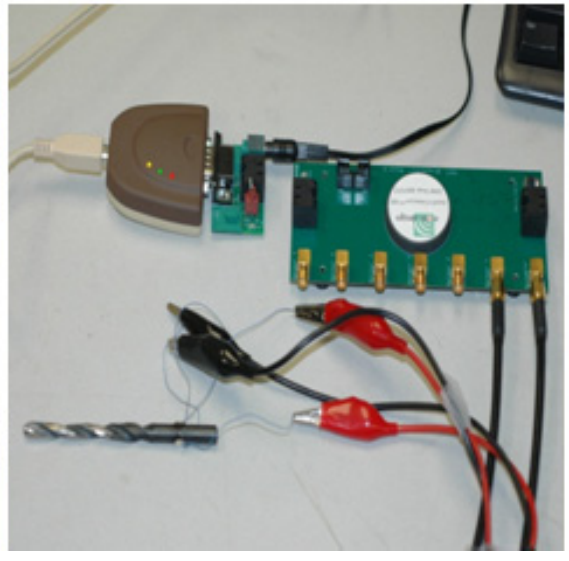

(b)

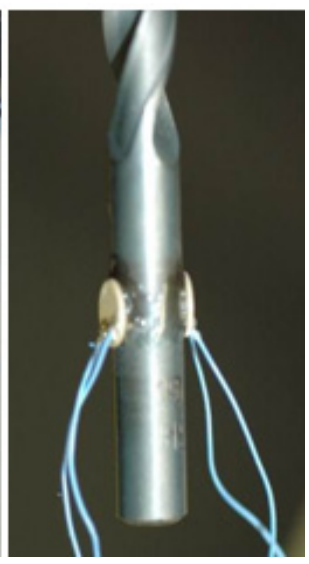

(c)

Figure 3.1 - Frequency response analysis experimental setup and specimen (a) experimental setup for frequency response analysis of drill-bit, (b) drill-bit equipped with Intelli-Connector MD7b data acquisition unit and (c) location of PWAS transducers on the specimen.

In order to keep the characteristics of piezoelectric elements, bonding material, solders and wires unchanged, the cutting edges of the drill were artificially worn by grinding them with a handheld rotary grinder. The new 
and artificially worn drills are shown in figure 3.2. The drill was grinded to four different wear levels: light, medium, severe and extreme.

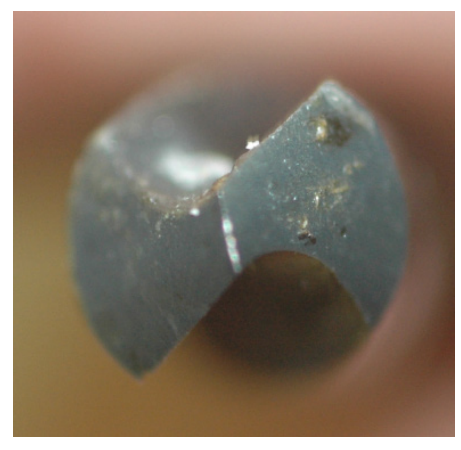

(a)

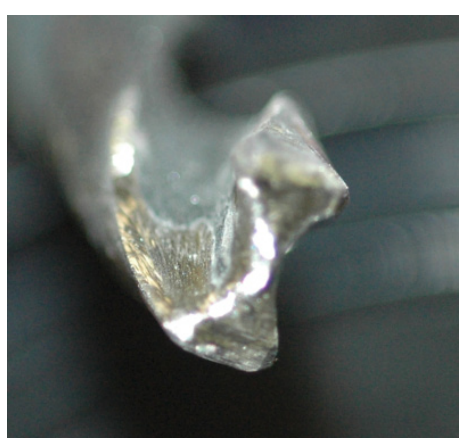

(b)

Figure 3.2 - High speed steel drill specimen; (a) new drill and (b) worn out drill

The wear levels can be seen in table1.To simulate the extreme wear, the tip of the drill is heavily grinded.The data of the new tool as well as after each wear levels was taken by using two different approaches: Sweep sine wave approach and lamb wave approach.

\begin{tabular}{|l|l|l|l|}
\hline & $\begin{array}{l}\text { Length of drill } \\
\text { bit }(\mathrm{mm})\end{array}$ & Diameter(mm) & $\begin{array}{l}\text { Grinded } \\
\text { length }(\mathrm{mm})\end{array}$ \\
\hline No cut & 111.92 & 7.49 & 0 \\
\hline First cut & 110.69 & 7.49 & 0 \\
\hline Second cut & 110.06 & 6.5 & 10.49 \\
\hline Third cut & 109.77 & 6.09 & 17.38 \\
\hline Fourth cut & 109.77 & 6.09 & 48.45 \\
\hline
\end{tabular}

Table 1 - Four different wear levels of drill bit.

\subsubsection{Surface response to excitation (SuRE)}

The experimental setup for this approach is shown in figure 2.7. The

Stanford Research Systems (SRS) Model SR780 signal analyzer wasused to 
excite one of the piezoelectric elements with sweep sine wave and the frequency response from the other piezoelectric element was obtained by the same equipment at 2000 data points in $0-100 \mathrm{KHz}$ range. The frequency and time domain signal was received by the instrument. The signal analyzer calculates the transfer functions in the frequency domain. The magnitude calculated for the new tool was used as reference. To see the difference between the signal received by the new and the worn out drill, sum of the squares of the differences was calculated. The variations of the calculated sum of squares were visualized using the graphs.

\subsubsection{Lamb wave approach}

The experimental setup for this approach is shown in the figure 3.1. To perform the experiment, Metis Design SHM Node MD 7502 was used to create the Lamb wave and to monitor their propagation. One of the piezoelectric elements is excited by a 3.5 sine wave with $200-300 \mathrm{KHz}$ of frequency. This signal was first pass through the Hanning window before being given to the piezoelectric element for excitation. The same system was used to measure the generated voltage by the other piezoelectric element bonded to the shank of the drill with $10 \mathrm{MHz}$ sampling frequency. The signal was collected at 8000 data points but only first 3000 of them were used. The received signals were averaged 64 times to minimize the noise contamination. The device used to perform the test is capable of 
synchronously facilitating $20 \mathrm{VPP}$ guided waves (GW), frequency response (FR) and acoustic emission (AE) testing up to $5 \mathrm{MHz}$ with $50 \mathrm{MHz} 12$ - bit data acquisition on 6 independent channels. The tests obtained by this method were time domain from the SHM Node MD 7052. The dominant frequency of the sampled signal was the same with the excitation signal. The delay time of each burst carries the information about tool geometry. Further after getting the data from the received signal, S-transformation was used to calculate the envelopes of the received signal. The significance of the differences among the envelopes was then evaluated by calculating the sum of the squares of the differences by following the same procedure outlined in the previous approach.

\subsection{Results and discussion}

The feasibility of two approaches was tested in this chapter. The performance of each approach is outlined as follows:

\subsubsection{Surface response to excitation}

The experimental data was gathered by testing the 19/64 inch diameter, high speed steel (HSS) drill bit. The data was taken for the new, and for other four wear levels, which are artificially created at the edges of the drill with a grinder. The magnitude of the transfer function was calculated once at each test condition and is shown in the figure 3.3. It can 
be seen from the graphs that the signals were similar but still some noticeable change in each case can be seen.
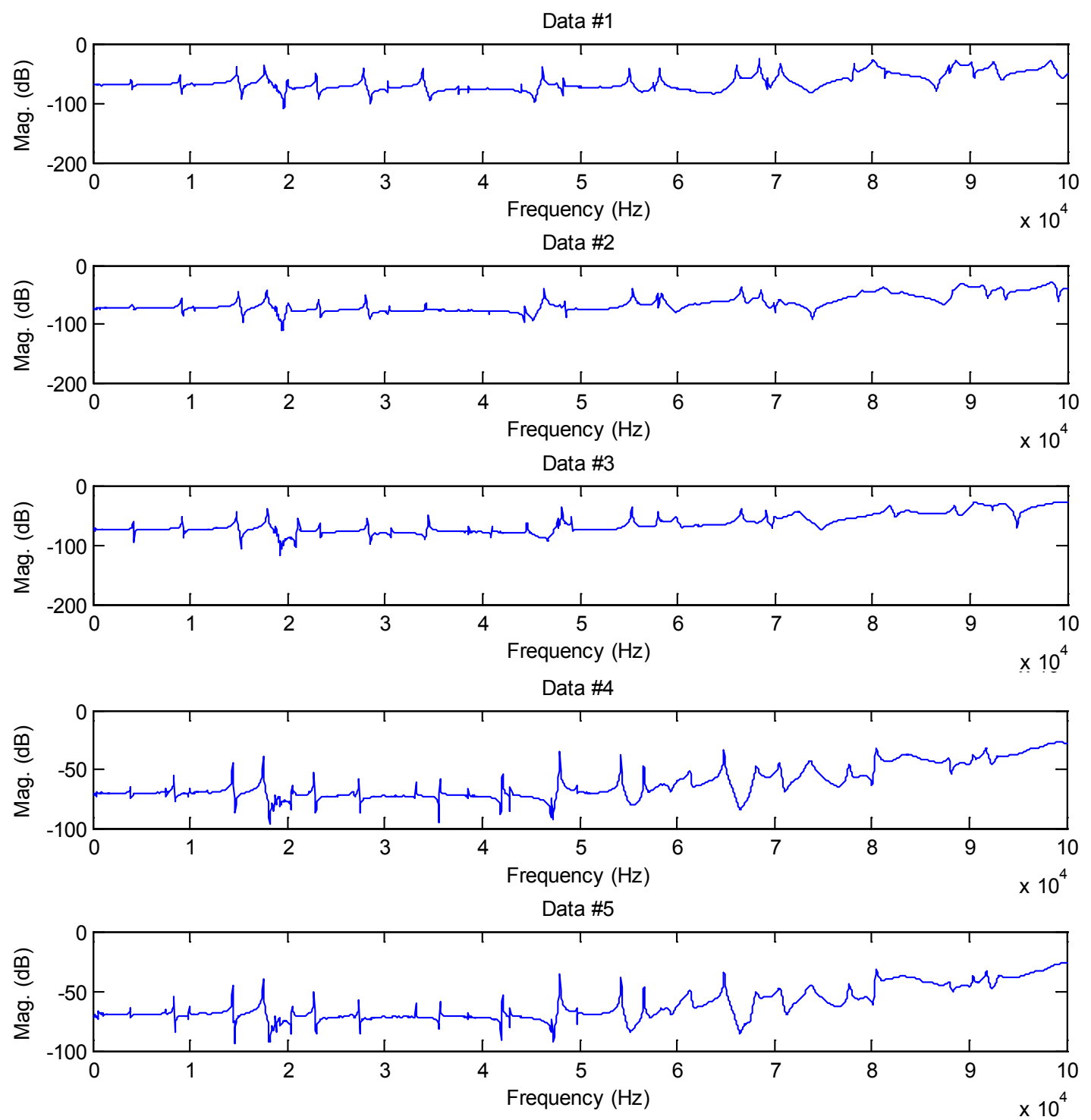

Figure 3.3 - Spectrum analyses of perfect and grinded tool at 4 different wear levels

The sum of the squares of the difference between each case is shown in

figure 3.4. In the figure the first bar graph is having a nil value because this bar represents the value for the perfect tool. The other bar graphs represents 
the difference between the perfect tool and the other four cases (for four different wear levels). The length of the bars for the sum of the squares of the new and the first three wear levels (light, medium and severe) increased significantly with almost equal steps. This pattern indicates that the characteristics of the magnitude changes with almost the same amount with increase of wear. It is though very difficult to separate the wear levels for the severe and extreme cases. The sum of the squares of the differences with respect to the case one was also studied at different frequency intervals, as it can be seen in figure 3.5. The difference between the five considered cases was observed best at $75-100 \mathrm{KHz}$ interval. Figure 3.5, suggests that the differences among the magnitudes are not very clear at two bandwidths below $50 \mathrm{KHz}$.

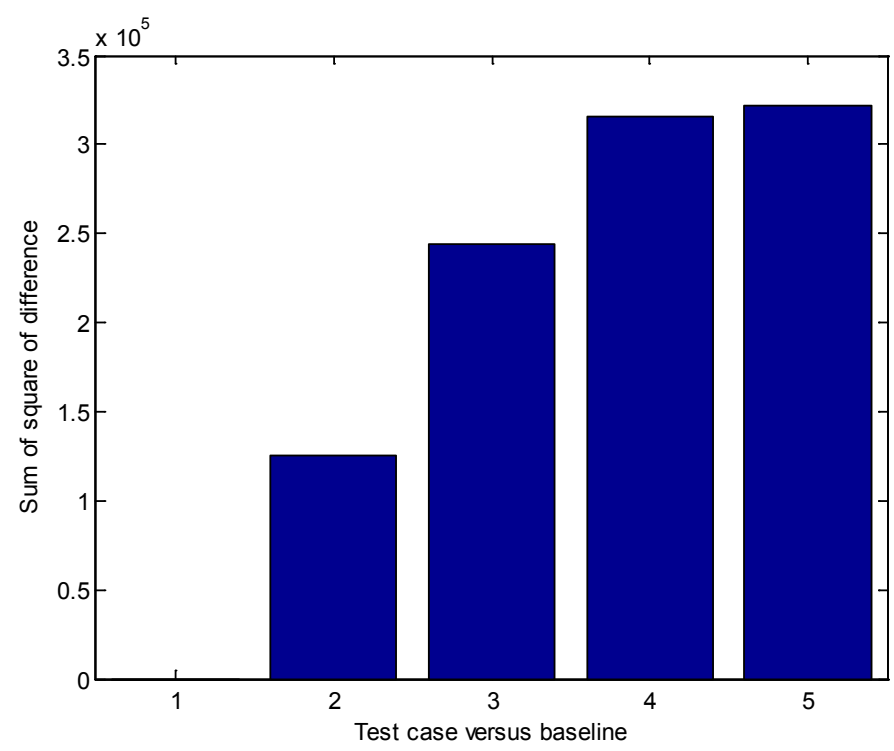

Figure 3.4 - The sum of the squares of the differences between the magnitude of each case and the reference. The first and the second cases are the perfect tool. The 3, 4 and 5 shows the cases for low, medium and significant wear. 


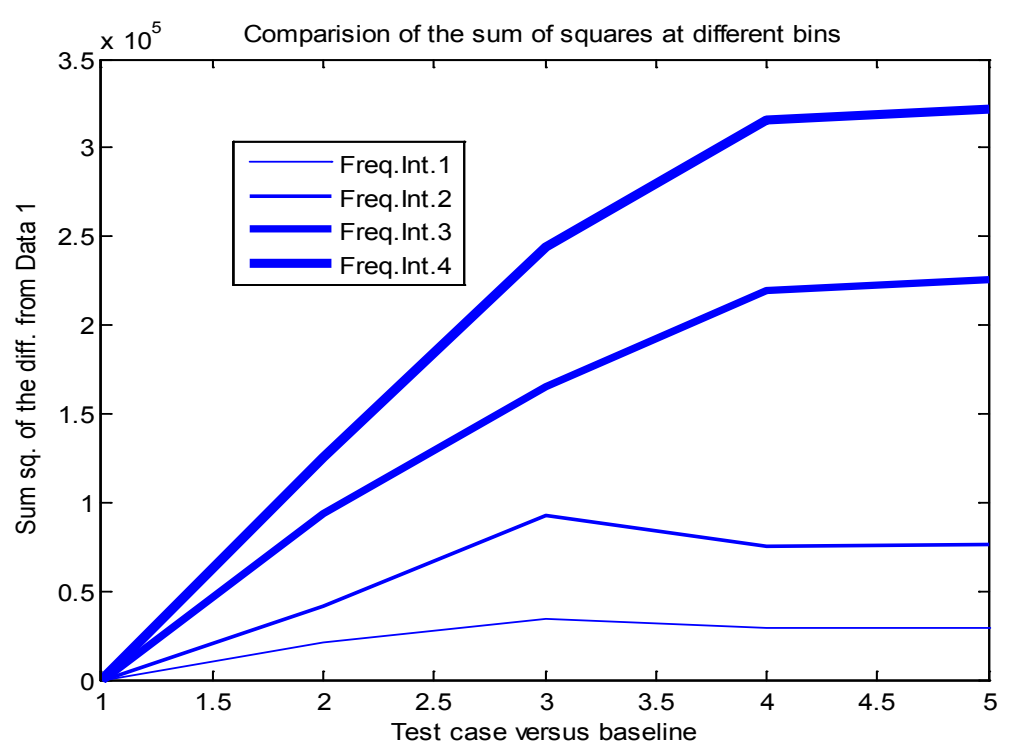

Figure 3.5 - The difference between the each test case and the perfect one at different frequency intervals. The frequency intervals designated 1 to 4 corresponds to $0 \mathrm{~Hz}$ to $25 \mathrm{KHz}, 25 \mathrm{KHz}$ to $50 \mathrm{KHz}, 50 \mathrm{KHz}$ to $75 \mathrm{KHz}$, and 75 $\mathrm{KHz}$ to $100 \mathrm{KHz}$ respectively.

\subsubsection{Lamb wave propagation}

The experimental data was collected by performing experiments on a HSS drill. The experiments were repeated at 200 and $300 \mathrm{KHz}$ excitation frequencies. The data was taken twice for the new and artificially worn tool. So, in all 12 sets of data was analyzed.

The voltage created by the piezoelectric element correlates with the power of the arriving Lamb waves. The signals received in the time domain looks very similar as shown in figure 3.6. 

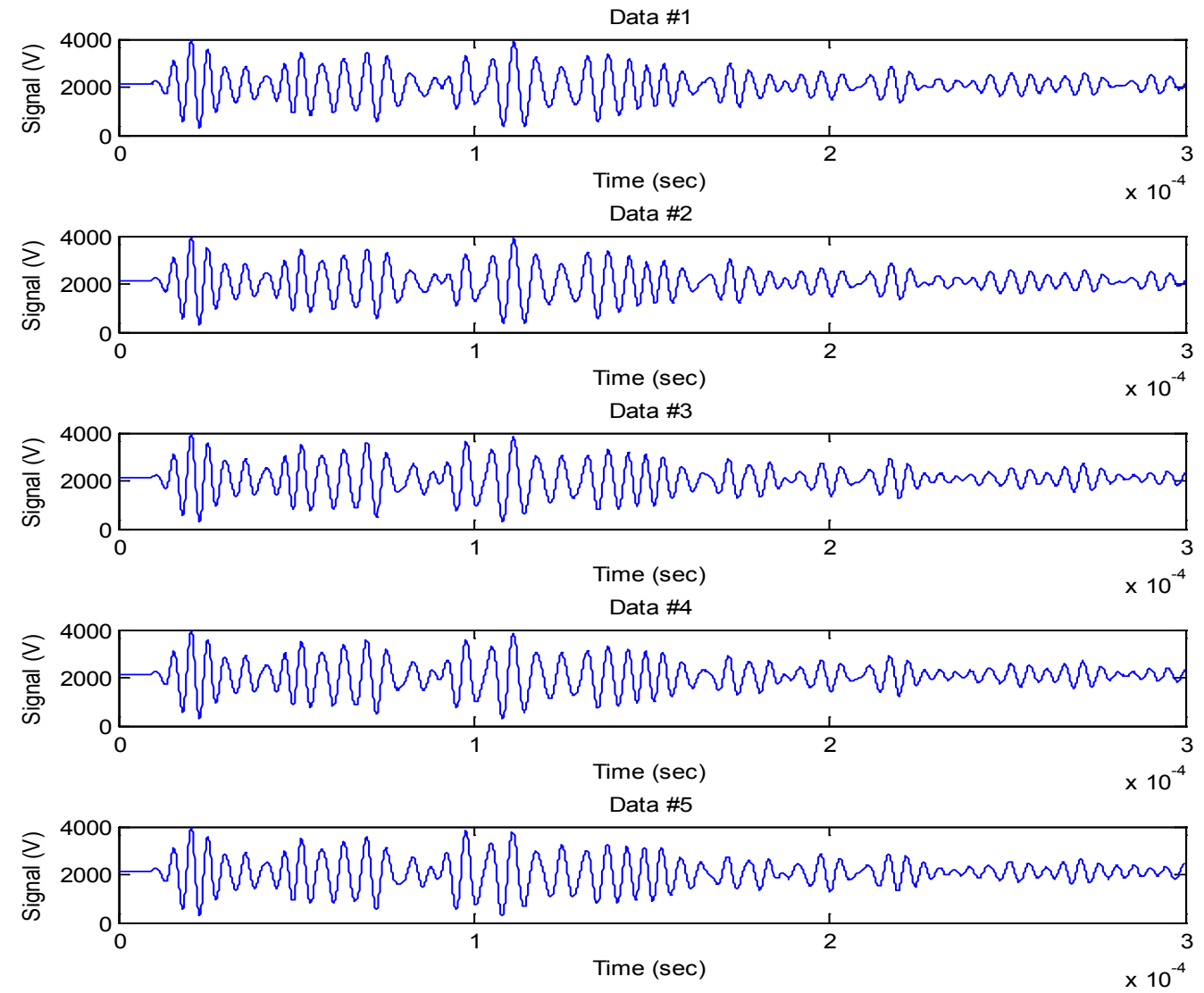

Figure 3.6 - The received signals by the sensing piezoelectric element.

The envelopes of the signals were calculated by using the $\mathrm{S}-$ transformation to identify the differences between the signals. The $\mathrm{S}-$ transformation of first 6 cases with $300 \mathrm{KHz}$ excitation frequency is shown in figure 3.7. The data 7 and 8 belongs to the new drill. The data 9-10 and 11-12 were collected when the drill undergoes light and medium wear respectively. 


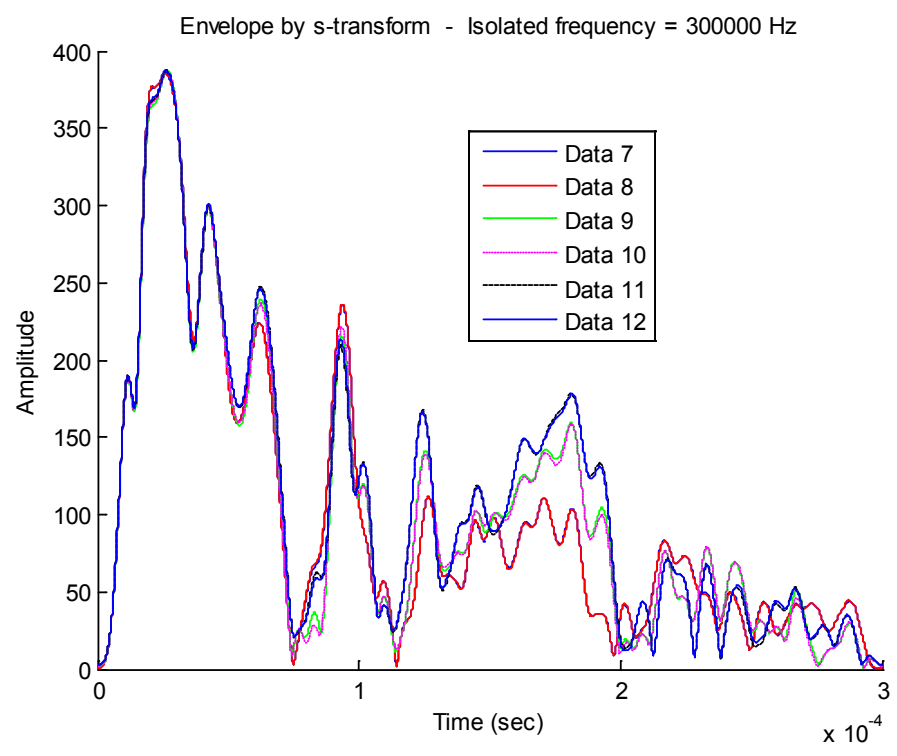

Figure 3.7 - The envelopes of the received signals by the sensing piezoelectric elements. The data numbered 7 and 8, 9 and 10, and 11 and 12 were obtained at the identical simulated wear levels, and they are almost the same. The first two belong to perfect (data 7,8), the following two corresponds to medium, and severe tool wear (data 11,12).

The envelopes were almost identical at the same wear levels. It can be seen from figure 3.8 and figure 3.9 that after $120 \mu \mathrm{sec}$, envelopes of the new, light and medium wear levels changes significantly. There is almost no difference between the envelopes in the first $75 \mu$ sec. The excitation frequencies used were $200 \mathrm{KHz}$ and $300 \mathrm{KHz}$ in figure 3.8 andfigure 3.9 respectively. 


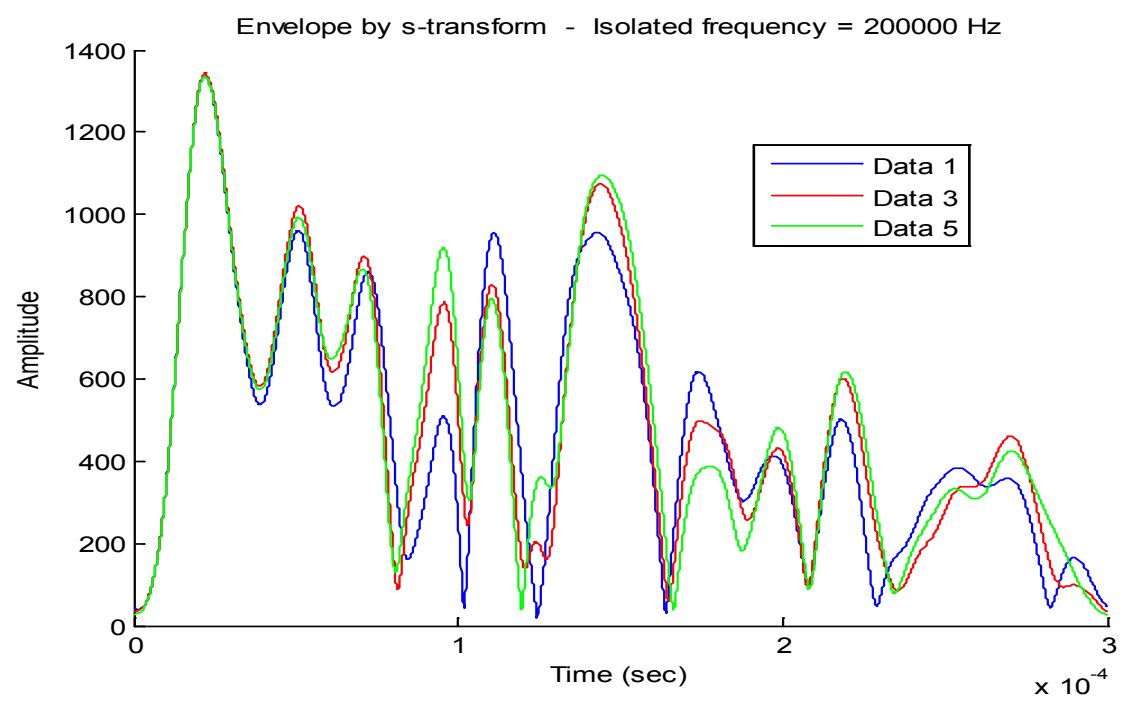

Figure 3.8 - Variation of the envelope signal with simulated tool wear when the $200 \mathrm{KHz} 3.5$ sine wave is applied. The perfect (Data 1), medium (Data 3) and severe (Data 5) simulated wear.

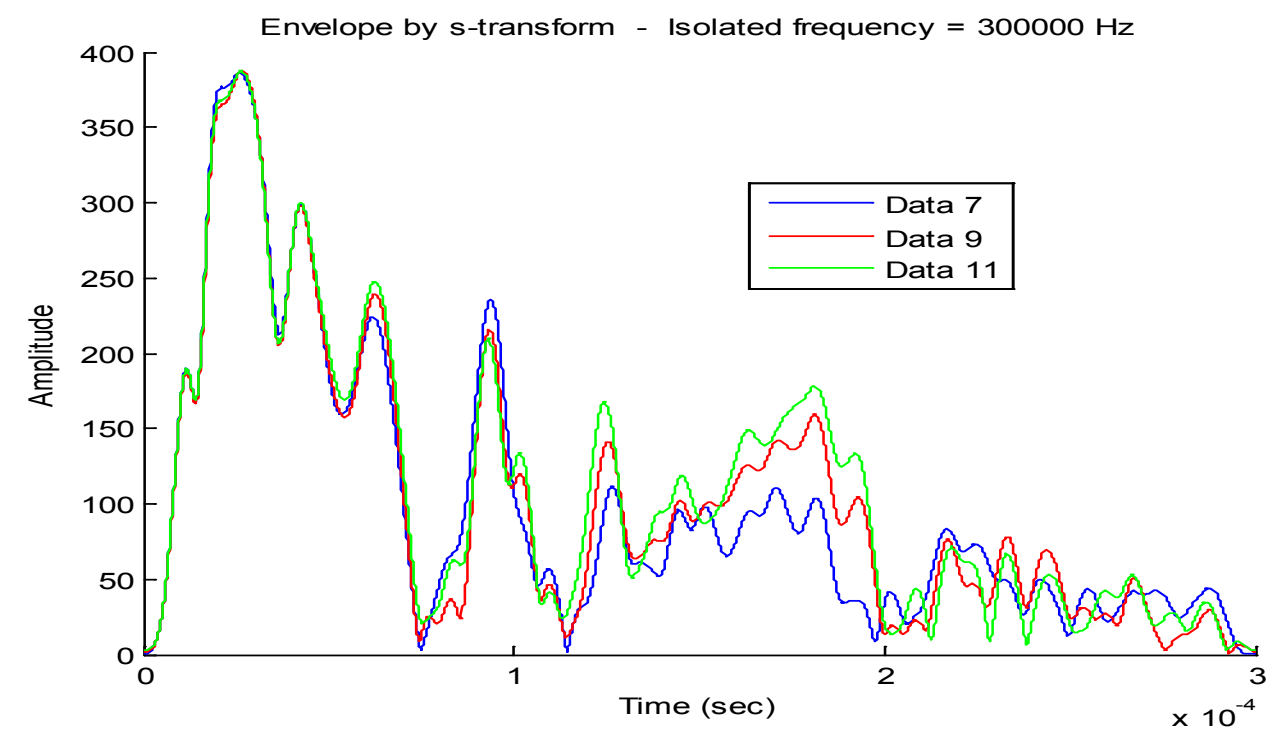

Figure 3.9 - Variation of the envelope signal with simulated tool wear when the $300 \mathrm{KHz} 3.5$ sine wave is applied. The perfect (Data 7), medium (Data 9) and severe (Data 11) simulated wear.

The sum of the squares of the differences of the envelopes between each case and the reference (envelope from the new drill) is plotted in figure 3.10 and figure 3.11 . The excitation frequencies for these cases were $200 \mathrm{KHz}$ 
and $300 \mathrm{KHz}$ and shown in figure 3.10 and figure 3.11 respectively.The four equally divided time intervals of considered $300 \mu$ sec long periods were automatically studied in these plots. The differences between the envelopes of the signals were most significant after $150 \mu$ sec. The new and two levels of wear could be detected from the sum of the squares of the difference of the envelopes in the $150-225 \mu \mathrm{sec}$, and $225-300 \mu$ sec time intervals with almost the same confidence.

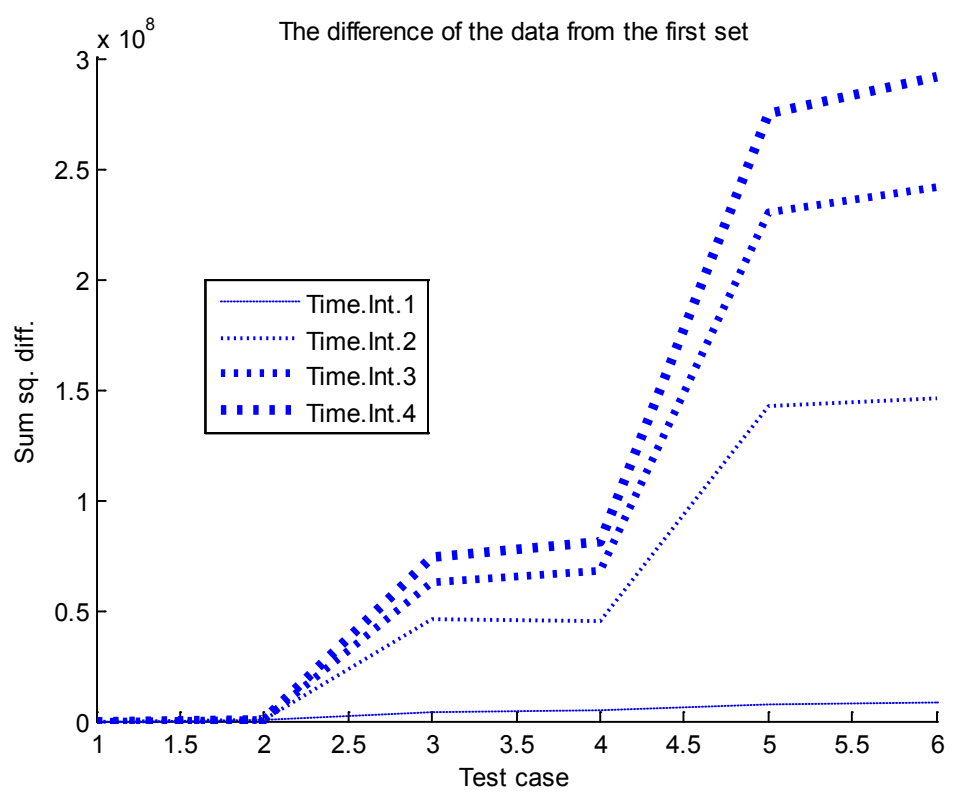

Figure 3.10 - The sum of the square of the difference between each set and the first one. The excitation signal frequency was $200 \mathrm{KHz}$. The data sets of 1 to 6 were tested. The intervals $1,2,3$, and 4 corresponds to the $0-75 \mu \mathrm{sec}$, 75-150 $\mu \mathrm{sec}, 150-225 \mu \mathrm{sec}$, and 225-300 $\mu$ sec respectively. 


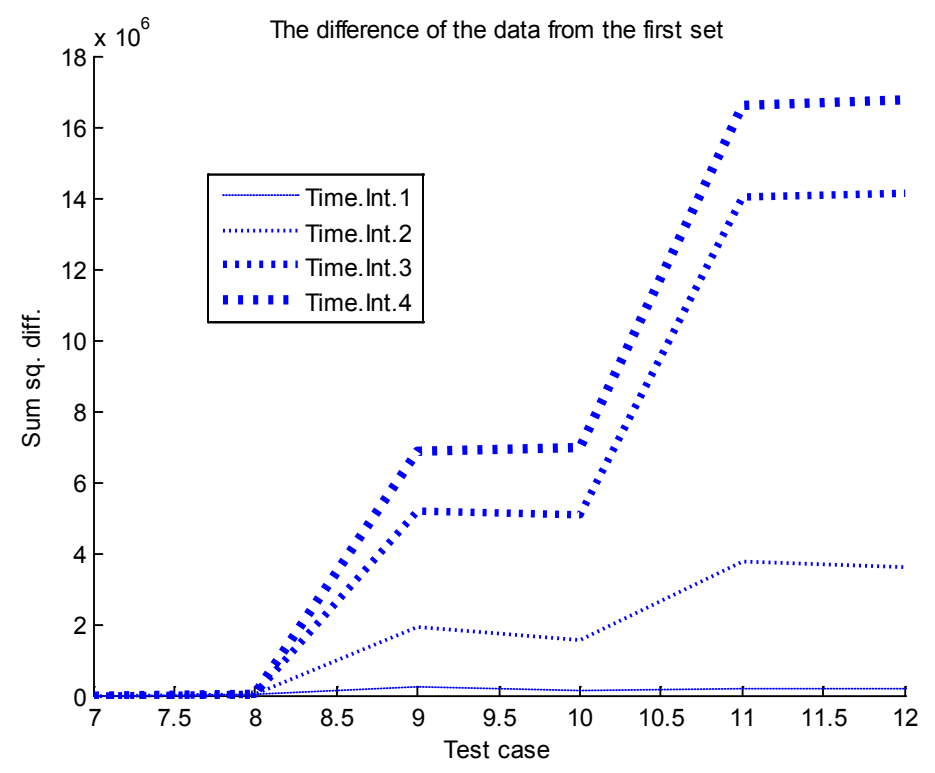

Figure 3.11 - The sum of the square of the difference between each set and the first one. The excitation signal frequency was $300 \mathrm{KHz}$. The data sets of 7 to 12 were tested. The intervals $1,2,3$, and 4 corresponds to the 0-75 $\mu \mathrm{sec}$, 75-150 $\mu$ sec, 150-225 $\mu \mathrm{sec}$, and 225-300 $\mu$ sec respectively.

\subsection{Conclusion}

Feasibility of the use of the structural health monitoring (SHM) methods was evaluated for estimation of the tool wear. The spectrum analysis and Lamb wave based approaches were successfully used to separate the signals of the new and worn out tool.

Spectrum analyzer conveniently estimated the transfer function of the surface response to excitation. Two piezoelectric elements were needed. Typically the costs of the spectrum analyzers are half to a quarter of impedance analyzers. The magnitude of the transfer function could be calculated with digital signal processers (DSP) without requiring any 
complicated electronics. However this approach requires bonding two piezoelectric elements.

Creation of the Lamb waves with one piezoelectric element and monitoring their propagation from the signal of the other piezoelectric element was straight forward. The hardware cost was the lowest among the two approaches discussed in this chapter. Two piezoelectric elements were needed. Calculation of the envelopes of the collected signals was necessary outside of the hardware. The $\mathrm{s}$ - transformation was used with this purpose and provided smooth envelope curves in a very consistent manner. Stransformation also isolates the noise from the signal, since the envelopes of only the harmonic component of the signal at the excitation frequency was obtained.

The study indicated that the SHM methods should also be considered when the next generation tool wear monitors are developed. Evaluation of the surface response to excitation with a spectrum analyzer and Lamb wave based approaches may be used together to complement each other for tool wear estimation. Both approaches use two piezoelectric elements bonded to the tool. 


\section{CHAPTER IV}

\section{CONCLUSION AND FUTURE WORK}

The Structural Health Monitoring (SHM) methods were introduced in the previous chapters and their feasibility was studied. The results confirmed that the SHM methods are capable of detecting any changes or defects in the structure. This chapter comprises of conclusions of all the above determined work followed by the future work.

\subsection{Conclusions}

A comprehensive procedure to locate and trace the intensity of defects in copper and carbon fiber tube based on time domain and frequency domain was presented.The proposed procedure was verified experimentally using two different pipes (copper and carbon fiber). The piezoelectric elements were used as actuators and sensors. These sensors were very light in weight and a lot cheaper than the conventional sensors. These sensors can be easily and permanently attached to the structures. The signals acquired from these sensors through two SHM methods were successfully refined to get the intelligible information. The intensity of defect in the copper pipe and carbon fiber tube was shown distinctively. Apart from this, it was shown that the strength of the signal attenuates over a longer traveling distance.

Monitoring the tool wear by same SHM techniques was also addressed.The S - transformation analysis and envelope of the S - 
transformation were used to extract the salient features representing a perfect and a worn tool from time domain signals. The defect intensity vectors for different tool states were identified using frequency domain analysis. The performance of both the SHM approaches was evaluated with four different wear levels and tool state detection was achieved successfully. The results indicate that these methods could be a successful tool for monitoring similar cutting tools.

The two SHM approaches used in this study were precisely tested, scrutinized and concluded. The equipment cost for lamb wave approach was the least of the two methods proposed. Only two sensors per test sample were required to perform the experiments for both approaches except for the long copper pipe. The overall cost of both the methods was less than the other available conventional SHM techniques.

\subsection{Future Work}

In this study, an exploratory study of two structural health monitoring methods for damage detection of round objects (pipes, tubes and cutting tool) is conducted. Some, observations are made during carrying out this study, and further research should be conducted to enhance the proposed damage detection methodology. The following recommendations are provided for future research considerations: 
- A chip level piezoelectric - wafer active sensor device possessing local processing ability and wireless communication ability must be developed to achieve in field in - situ SHM applications.

- In order to treat the general problem of buried pipes, the effect of a solid medium outside the pipe has to be investigated.

- Number of sensors can be reduced by using more sophisticated sensors capable of directional sweeping larger areas without touching the piezoelectric sensors.

- More research on different kind of damage at different locations using the proposed damage detection approach and sensor system should be conducted.

- Further utilization of numerical FE simulation may simplify the experimental study and help guide the location of the sensors to achieve even better results. 


\section{REFERENCES}

1. L.F. Zou, G.A. Ferrier, V. ShahraamAfshar, and X.Y. Bao, "Structural health monitoring of pipelines with distributed brillouin sensor," Structural Health Monitoring and Intelligent Infrastructure, Vol. 1.

2. A. Tododroki, Y. Takeuchi, Y. Shimamura, A. Iwasaki, T. Sugiya, "Fracture Monitoring System of Sewer Pipe with Composite Fracture Sensors Via the Internet," Structural Health Monitoring, Vol. 3, N0. 1, 5-17, 2004.

3. V. Giurgiutiu and A.N. Zagrai, "Characterization of Piezoelectric Wafer active Sensors," Journal of Intelligent Material Systems and Structures, Vol. 11 December 2000.

4. V. Giurgiutiu, "Embedded NDE with Piezoelectric Wafer-Active Sensors in Aerospace Applications," Journal of Materials (JOM), Online special issue on Nondestructive Evaluation, January 2003.

5. A. Demma, P. Cawley, M. Lowe, A.G. Roosenbrand, B. Pavlakovic, "The reflection of guided waves from notches in pipes: a guide for interpreting corrosion measurements," NDT\&E International 37, 167-180, 2004.

6. L. Yu and V. Giurgiutiu, "Multi-mode Damage Detection Methods with Piezoelectric Wafer Active Sensors," Journal of Intelligent Material Systems and Structures, Vol. 20, July, 2009.

7. V. Giurgiutiu, B. Xu and W. Liu, "Development and Testing of Hightemperature Piezoelectric Wafer Active Sensors for Extreme Environments," Structural Health Monitoring, March 23, 2010.

8. H. Huang, T. Pamphile and M. Derriso, "Directionality of Lamb Wave Displacement Field Generated by Circular Piezoelectric Wafer Actuators," Structural Health Monitoring, Vol. 1, No. 1088-1095, 2009.

9. H. Sohn, H. W. Park, K.H. Law, and C.R. Farrar, "Damage Detection in Composite Plates by Using an Enhanced Time Reversal Method," Journal of Aerospace Engineering, ASCE, 2004.

10. S.S. Kessler, S Mark Spearing and C. Soutis, "Damage detection in composite materials using Lamb wave methods," Smart Mater. Struct. 11:269-278, 2002 . 
11. N. O'Donoughue, J. Harley, J.M.F. Moura and Y. Jin, "Detection of Structural Defects in Pipes using Time Reversal of Guided Waves," Proceedings 43rd Asilomar Conference on Signals, Systems, and Computers.

12.M.J.S. Lowe, D.N.Alleyne, P.Cawley, "Defect detection in pipes using guided waves," Ultrasonics 36:147-154, 1998.

13. S. Park, C.B. Yun, Y. Roh and J.J. Lee, "PZT-based active damage detection techniques for steel bridge components," Smart Mater. Struct. 15:957-966, 2006.

14.V. Giurgiutiu and Y. Lingyu. "Embedded Ultrasonic Structural Radar with Piezoelectric Wafer Active Sensors for Damage Detection in Cylindrical Shell Structures," 45th AIAA/ASME/ASCE/AHS/ASC Structures, Structural Dynamics \& Materials Conference and 12th AIAA/ASME/AHS Adaptive Structures Forum, Palm Springs, CA, 19-22 Apr. 2004, paper \# AIAA-20041983.

15.V. Giurgiutiu, "Lamb Wave Generation with Piezoelectric Wafer Active Sensors for Structural Health Monitoring," SPIE's 10th Annual International Symposium on Smart Structures and Materials and 8th Annual International Symposium on NDE for Health Monitoring and Diagnostics, 26 March 2002, San Diego, CA. paper \# 5056-17.

16.X. Wang and I. N. Tansel, "Modeling the Propagation of Lamb Waves using a Genetic Algorithm and S-transformation," Structural Health Monitoring, 2537, 2007.

17.M. fink and k. ros, "time reversed lamb waves," IEEE transactions on ultrasonics, ferroelectrics, and frequency control, vol.45, no.4, July 1998.

18. PHMSA stakeholder communications: pipeline and hazardous materials safety administration U.S. department of transportation, 2009

19. M. Afshari, S. Park and D. J. Inman, "The early stage crack detection using non-linear feature extraction of the self-sensing piezoelectric impedance measurements," The 7th International Workshop on Structural Health Monitoring, 1885-92, 2009.

20.Tseng, K.K-H., and A.S.K. Naidum, "Non-parametric damage detection and characterization using smart piezoceramic material," Smart Materials and Structures, 11:317-329, 2002. 
21.Lalande, C.A and Rogers, "Solid-State Active Sensing for in-situ Health Monitoring," Proceedings, Society for Machinery Failure Prevention Technology Showcase, Mobile, AL, April 22-26, 1996.

22.Lalande, B. Childs, Z. Chaudhry, and C.A. Rogers, "High-Frequency Impedence Analysis for NDE of Complex Precision Parts," Proceedings, SPIE Conference on Smart Structures and Materials, San Diego, CA, 26-29 February, SPIE Publishing, Bellingham, WA, 2717, pp. 237-245, 1996.

23.Ayres, F. Lalande, Z. Chaudhry, and C.A. Rogers, "Qualitative Health Monitoring of a Steel Bridge Structure via Piezoelectric Actuator/Sensor Patches," Proceedings, SPIE Nondestructive Evaluation Techniques for Aging Infrastructure \& Manufacturing, Scottsdale, AZ, 2-5 December, 2946, pp. 211-218, 1996.

24.D. C. Price, D. A. Scott, G. C. Edwards, A. Batten, A. J. Farmer, M. Hedley, M. E. Johnson, C. J. Lewis, G. T. Poulton, M. Prokopenko, P. Valencia, P. Wang, "An Integrated Health Monitoring System for an Ageless Aerospace Vehicle", 4th International Workshop on Structural Health Monitoring, Stanford University, USA.

25.D. Parekh and A. Sinha, "Recent Applications of Health and Usage Monitoring Systems to Rotocraft - A Survey", AIAC12 - Twelfth Australian International Aerospace Congress, Australia.

26.S. Willis, "SHM: A Hands on Approach", Structural Health Monitoring Volume 1, 67-74, 2009.

27.PennWell Corporation, Oil and Gas Journal: Transportation Special Report (Houston, TX), p. 76 and similar table in earlier editions, 2001-08.

28.D. Thomas, J. Welter, and V.Giurgiutiu, "Corrosion Damage Detection with Piezoelectric Wafer Active Sensors”, Smart Mater. Struct. 11:957-66, 2004.

29. T. Stepinski and M. Engholm, "Piezoelectric Circular Array for Structural Healtm Monitoring Using Lamb Waves", The 7th International Workshop on Structural Health Monitoring, 1050-56, 2009.

30. S. Bhalla. A. Gupta, S. Bansal and T. Garg, "Ultra Low-Cost Adaptations of Electro-mechanical Impedence Technique for Structural Health Monitoring", Journal of Intelligent Material Systems and Structures, Vol. 20 - May 2009. 
31.V. Giurgiutiu, J. Bao and W. Zhao, "Piezoelectric wafer active sensor embedded ultrasonics in beams and plates", Exp. Mech. 43: 428-449, 2003.

32. W. Qu, L. Xiao and Y. Zhou, "Finite Element Simulation of Lamb Wave with Piezoelectric Transducers for Plasticity- Driven Damage Detection", Advanced Materials Research Vols. 79-82, pp. 1095-1098, 2009.

33.S.S. Kessler, "Piezoelectric-Based In-Situ Damage Detection of Composite Materials for Structural Health Monitoring Systems", PhD thesis, Massachusetts Institute of Technology, 2002.

34. Workman, L. Gary and Kishoni, Doron, Moore, O. Patrick, "Nondestructive Testing Handbook," Third edition: Volume 7, ultrasonic testing, 100-103, 2007.

35.J. H. Nieuwenhuis, J. Neumann, D.W. Greve, and I.J. Oppenheim, “ Generation and detection of guided waves using PZT wafer transducers", Ultrasonics, Ferroelectrics and Frequency Control, Vol. 52 Issue-11, 2103$2111,2005$.

36. J. Li and J.L. Rose, "Excitation and propagation of non-axisymmetric guided waves in a hollow cylinder," J. Acoust. Soc. Am. Volume 109, Issue 2, pp. 457464, February 2001.

37. Gazis, D.C., "Three-dimensional investigation of the propagation of waves in hollow circular cylinders," J.Acoust.Soc.Am.31, 568-578, 1959.

38. S. Djili and F. Boubenider, "Propagation of guided waves in a hollow circular cylinder application to non-destructive testing," EPJ Web of Conferences 6, 15004, 2010.

39. M.G. Silk and K.F.Bainton, "The propagation in metal tubing of ultrasonic wave modes equivalent to lamb waves," Ultrasonics 17, 11-9, 1979.

40. Stockwell, R. G., L. Mansinha, and R. P. Lowe, "Localization of the Complex Spectrum: The S Transform," IEEE Transactions on Signal Processing, Vol.44, No. 4, pp.998-1001, 1996

41.Pakrashi, V., and B. Ghosh, "Application of S Transform in Structural Health Monitoring," NDTCE'09, Non-Destructive Testing in Civil Engineering, Nantes, France, 2009 
42. Wang, X., and I. N. Tansel, "Modeling the Propagation of Lamb Waves using a Genetic Algorithm and S-transformation," Structural Health Monitoring, Vol.6, No.1, pp. 23-37, 2007

43. Stockwell, R. G., "Why use the S-Transform," Mathematics Subject Classification, 1991.

44.D. Kerr, J.Pengilley and R. Garwood, "Assessment and visualization of machine tool wear using computer vision," The International Journal of Advanced Manufacturing Technology, Volume 28, Numbers 7-8, 781-791.

45.R. Schmitt, R. Hermes, M. Stemmer, A. Pavim, F. Deschamps, D. Moraes, "Machine Vision Prototype for Flank Wear Measurement on Milling Tools," Proceedings of 38th CIRP - International Seminar on Manufacturing Systems (2005), pp. 123-134.

46. M. Trujillo, W. Li, B.Fallerio, E. Paz and I.Tansel, "Inspection of micro-tools at high rotational speeds," International Journal of Machine Tools and Manufacture, Volume 34, Issue 8, November 1994, Pages 1059-1077

47.W. Y. Bao and I.N. Tansel, "Modeling micro-end-milling operations: I. Analytical cutting force model," International Journal of Machine Tools and Manufacture, Volume 40, Issue 15, December 2000, Pages 2155-2173

48.W.Y. Bao, N.S. Reen and C.V. Kropas-Hughes, "Genetic tool monitor (GTM) for micro-end-milling operations," international Journal of Machine Tools and Manufacture, Volume 45, Issue 3, March 2005, Pages 293-299.

49. Y.Xu, Kumehara, "Radial Basis Function Network Based Monitoring of Tool Wear States," Second International Symposium on Computational Intelligence and Design, 2009, Issue Date: 12-14 Dec. 2009, pp. 521 - 524 .

50. I. N. Tansel, M. Li, M. Demetgul, K. Bickraj, B. Kaya and B. Ozcelik, "Detecting chatter and estimating wear from the torque of end milling signals by using Index Based Reasoner (IBR)," The International Journal of Advanced Manufacturing Technology, DOI: 10.1007/s00170-010-2838-5 Currently on-line, in print.

51.G. D'errico and G. Rutelli," Tool wear monitoring based on cutting power measurement," Wear Volume 139, Issue 2, August 1990, Pages 303-311. 
52. G. Park, H. Sohn, C.R. Farrar and D. J. Inman, "Overview of Piezoelectric Impedance-Based Health Monitoring and Path Forward," The Shock and Vibration Digest, Vol. 35, No. 6, November 2003 451-463.

53. V.Giurgiutiu, Structural Health Monitoring with Piezoelectric Wafer Active Sensors, Academic Press, 2007.

54.D. M. Peairs, G. Park, D. J. Inman, "Improving Accessibility of the Impedance-Based Structural Health Monitoring Method," Journal of Intelligent Material Systems and Structures, vol. 15, no. 2, 129-139, February 2004.

55.BuliXu, V.Giurgiutiu, "A Low-Cost and Field Portable Electromechanical (E/M) Impedance Analyzer for Active Structural Health Monitoring," Structural Health Monitoring, Edited by Fu-Kuo Chang, pp.634-644, 2005.

56.W. Mohr and P. Holler, "On inspection of thin walled tubes for transverse and longitudinal flaws by guided ultrasonic waves," IEEE Trans. Sonics Ultrasonics 23, 369-374, 1976.

57.G.A. Alers, "Applications of special wave modes to industrial inspection problems, Proc. ASME winter meeting, Symposium on wave propagation and emerging technologies, Chicago, IL, 1994.

58.D.N. Alleyne and P. Cawley, "The excitation of Lamb waves in pipes using dry coupled piezoelectric transducers," J. NDE 15, 11-20, 1996.

59. Forte carbon fiber products, (www.forterts.com), October, 2010. 